\title{
Estabilidade de Mittag-Leffler e aplicações às redes neurais de Hopfield fracionárias
}

Bruno Borges de Souza

\author{
DisSERTAÇÃO APRESENTADA \\ $\mathrm{AO}$ \\ Instituto de Matemática e Estatística \\ DA \\ Universidade De SÃo Paulo \\ PARA \\ OBTENÇÃO DO TÍTULO \\ $\mathrm{DE}$ \\ Mestre em CiênCias \\ Programa: Matemática Aplicada \\ Orientador: Prof. Dr. Pedro Aladar Tonelli
}

Durante o desenvolvimento deste trabalho o autor recebeu auxílio financeiro da CAPES

São Paulo, Julho de 2019 


\section{Estabilidade de Mittag-Leffler e aplicações às redes neurais de Hopfield fracionárias}

Esta versão da dissertação contém as correções e alterações sugeridas pela Comissão Julgadora durante a defesa da versão original do trabalho, realizada em 12/09/2019. Uma cópia da versão original está disponível no Instituto de Matemática e Estatística da USP. 


\section{Agradecimentos}

Meus agradecimentos especiais aos meus pais que me apoiaram e me incentivaram nesta jornada e por sua dedicação.

Ao Professor Pedro Tonelli, que desde da época de minha iniciação científica compartilhou seu

conhecimento que me incentivou a apreciar ainda mais a matemática, que muito contribuiu para a finalização desta dissertação.

Aos membros da Comissão Julgadora pelos conselhos e sugestões que enriqueceram muito este trabalho.

Agradeço também à CAPES, pelo suporte financeiro oferecido. 


\section{Resumo}

Borges de Souza, B. Estabilidade de Mittag-Leffler e aplicações às redes neurais de Hopfield fracionária. 2019. 94 f. Dissertação (mestrado) - Instituto de Matemática e Estatística, Universidade de São Paulo, São Paulo, 2019.

Neste trabalho estudamos um critério para a estabilidade de Mittag-Leffler dos pontos de equilíbrio de um sistema de equações diferenciais ordinárias com derivadas fracionárias de Caputo e Riemann-Liouville. Como um exemplo de aplicação, estudamos um critério para estabilidade assintótica dos pontos de equilíbrio de uma rede neural de Hopfield fracionária.

Palavras-chave: Redes Neurais, Cálculo fracionário, estabilidade de Mittag-Leffler. 


\section{Abstract}

Borges de Souza, Bruno. Mittag-Leffler stability and applications to fractional Hopfield neural networks. 2019. 94 f. Dissertation (master) - Instituto de Matemática e Estatística, Universidade de São Paulo, São Paulo, 2019.

In this work we shall study a criterion for the Mittag-Leffler stability of the equilibrium points of a system of ordinary differential equations with fractional derivatives of Caputo and RiemannLiouville. As an example of application, we study a criterion for asymptotic stability of the equilibrium points of a fractional Hopfield neural networks.

Keywords: Neural Network, Fractional calculus, Mittag-Leffler stability. 


\section{Sumário}

\begin{tabular}{ll}
\hline Lista de Símbolos & ix
\end{tabular}

\begin{tabular}{lll}
\hline 1 & Introdução & 1
\end{tabular}

2 Cálculo Fracionário $\quad 3$

2.1 Função Gama . . . . . . . . . . . . . . . . . . . . . . . . . . . . . . . . . 3

2.2 Integral e derivada de Riemann-Liouville $\ldots \ldots \ldots \ldots \ldots \ldots$

2.3 Derivada de Caputo . . . . . . . . . . . . . . . . . . . . 8

2.4 Derivada de Caputo vs Riemann-Liouville $\ldots \ldots \ldots \ldots$. . . . . . . . . . . . 9

2.5 Uma Interpretação física do Cálculo Fracionário . . . . . . . . . . . . . . . . . . . . 14

$2.5 .1 \quad$ Interpretação dos extremos (limites à esquerda) $\ldots \ldots \ldots$. . . . . . . . . 15

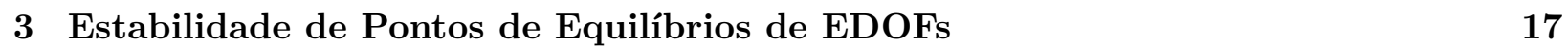

3.1 Função de Mittag-Leffler . . . . . . . . . . . . . . . . . . . . . 17

3.2 Transformada de Laplace $\ldots \ldots \ldots$. . . . . . . . . . . . . . . . . . . 18

3.3 Transformada de Laplace para o cálculo fracionário . . . . . . . . . . . . . . . . . . . 20

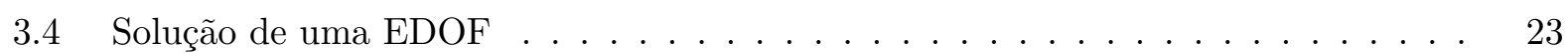

$3.5 \quad$ Existência e Unicidade de EDOFs . . . . . . . . . . . . . . . . . . . . . . . 24

3.6 Estabilidade de Lyapunov $\ldots \ldots \ldots$

3.6 .1 Método de Lyapunov $\ldots \ldots \ldots \ldots$. . . . . . . . . . . . . . . . . . . . . . . . . . . . . .

3.6 .2 Princípio da Invariância de LaSalle . . . . . . . . . . . . . . . . . . 37

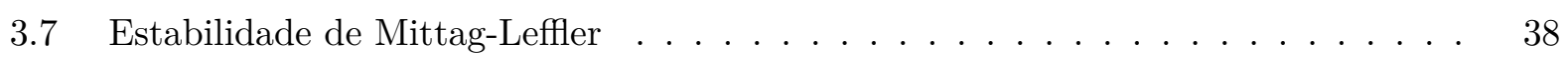

$3.7 .1 \quad$ Conceito de ponto de equilíbrio para sistemas de ordem fracionária . . . . 38

$3.7 .2 \quad$ Estabilidade de Mittag-Leffler . . . . . . . . . . . . . . . . . 39

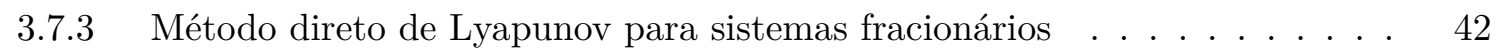

\begin{tabular}{|lll|}
\hline 4 & Redes Neurais Artificiais de Hopfield Fracionárias & $\mathbf{4 7}$
\end{tabular}

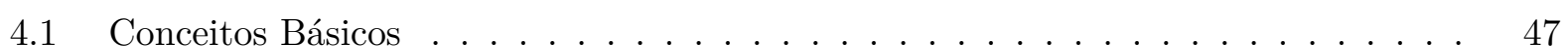

$4.2 \quad$ Redes Neurais de Hopfield Fracionárias (FHNN) $\ldots \ldots \ldots$. . . . . . . . . . . 52

4.3 Estabilidade de Hopfield $\ldots \ldots \ldots \ldots \ldots$

4.4 Algumas aplicações das Redes Neurais de Hopfield fracionárias . . . . . . . . . . . 55

\begin{tabular}{|lll}
5 & Estabilidade de Hopfield em Redes Neurais Fracionárias & $\mathbf{5 7}$
\end{tabular}

5.1 Um exemplo de critério de estabilidade para as Redes de Hopfield. . . . . . . . . . 58

5.2 Estabilidade de Mittag-Leffler para FHNNs $\ldots \ldots \ldots \ldots$. . . . . . . . . . 60 
viii SUMÁRIO

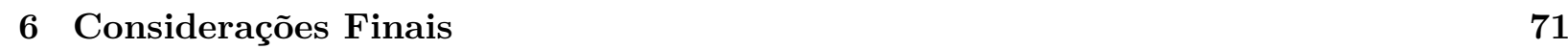

\begin{tabular}{|ll}
\hline A Teoremas de Ponto Fixo & 73
\end{tabular}

\begin{tabular}{lll}
\hline B Aplicação no Matlab & 75
\end{tabular} 


\section{Lista de Símbolos}

${ }_{a} \mathrm{~J}_{x}^{\alpha} f(x) \quad$ Integral de Riemann-Liouville de ordem $\alpha$ com relação a $x$ e terminal $a$ de $f(x)$

${ }_{a}^{\mathrm{RL}} \mathrm{D}_{x}^{\alpha} f(x) \quad$ Derivada de Riemann-Liouville de ordem fracionária $\alpha$ com relação a $x$ e terminal inferior $a$ de $f(x)$

${ }_{a}^{\mathrm{C}} \mathrm{D}_{f}^{\alpha}(x) \quad$ Derivada de Caputo de ordem fracionária $\alpha$ com relação a $x$ e terminal inferior $a$ de $f(x)$

${ }_{a} \mathrm{D}_{x}^{\alpha} f(x) \quad$ Derivada de Caputo ou Riemann-Liouville, de ordem fracionária $\alpha$ com relação a $x$ e terminal inferior $a$ de $f(x)$

$D f(x) \quad$ Derivada de ordem inteira da função $f(x)$

$\nabla^{(\alpha)} f(x)$ gradiente fracionário com derivada de Caputo de uma função escalar com respeito à variável $x=\left(x_{1}, x_{2}, \ldots, x_{n}\right)$

$E_{\alpha}(x) \quad$ Função de Mittag-Leffler de um parâmetro

$E_{\alpha, \beta}(x) \quad$ Função de Mittag-Leffler de dois parâmetros

$\Gamma(x) \quad$ Função Gama de $x$

$\mathcal{L}(f(x)) \quad$ Transformada de Laplace da função $f(x)$ 


\section{Lista de Figuras}

2.1 Função gama no intervalo $[-5,5] \ldots \ldots \ldots \ldots$. . . . . . . . . . . 4

$\left.\begin{array}{lll}2.2 & \text { As derivadas à esquerda e à direita como operações no passado e futuro de } \mathrm{f}(\mathrm{t})\end{array}\right]$

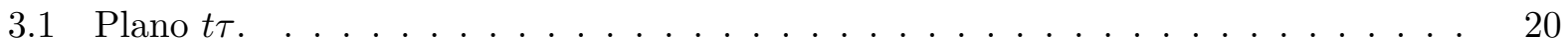

3.2 Noção geométrica de estabilidade em duas dimensões. . . . . . . . . . . . . . . . . 34

3.3 Noção geométrica de estabilidade assintótica em duas dimensões. . . . . . . . . . . . . 34

4.1 A anatomia de um neurônio $\ldots \ldots \ldots \ldots$

4.2 Ligação entre neurônios pós e pré sinápticos. Fonte: [51]. . . . . . . . . . . . . . 49

4.3 Modelagem de um neurônio por um circuito RC. . . . . . . . . . . . . . . . . . 49

4.4 Modelo de um rede neural artificial. . . . . . . . . . . . . . . . . . . . . . 50

4.5 Gráfico da função sigmoide. . . . . . . . . . . . . . . . . . . . . 51

4.6 Modelo de uma rede neural de Hopfield. . . . . . . . . . . . . . . . . . . . . 51

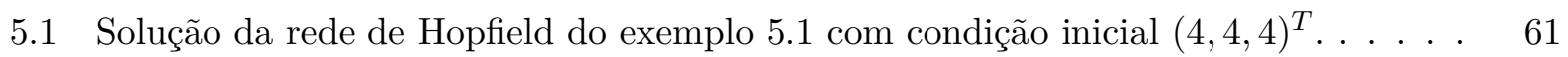

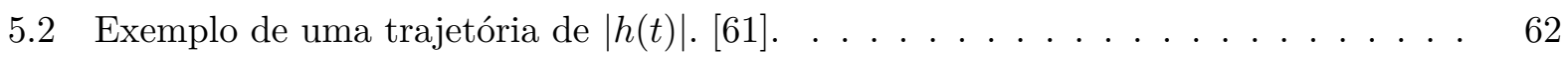

5.3 Exemplo de uma trajetória de $|h(t)|$. Fonte: 61. . . . . . . . . . . . . . . . . . 62

\begin{tabular}{|ll|l|l|l|}
\hline 5.4 & Solução da FHNN & 5.5 & com condição inicial $(4,4,4)^{T}$ para os valores de $\alpha=0.9,0.8$, \\
\hline
\end{tabular}

$0.7,0.6$ e 0.5 em cada uma das coordenadas de $v(t)=\left[v_{1}(t), v_{2}(r), v_{3}(t)\right]^{T}$. . . . . 68

5.5 Solução da FHNN com condição inicial $(4,4,4)^{T}$ e constantes externas dependentes do tempo para os valores de $\alpha=0.9,0.8,0.7,0.6$ e 0.5 em cada uma das coordenadas

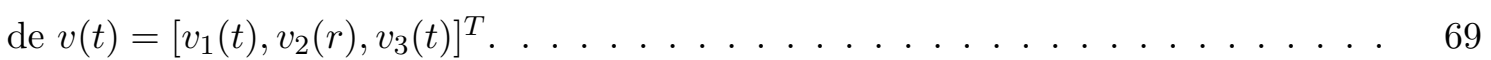




\section{Capítulo 1}

\section{Introdução}

O estudo das redes neurais artificias existe há mais de 70 anos, iniciando em 1943, quando McCulloch and Pitts [41] introduzem as redes neurais como máquinas computacionais. A ideia do neurônio de McCullosh-Pitts é criar duas entradas, que podem ser excitatórias $(+1)$, ou inibitórias $(-1)$. A função de ativação irá multiplicar cada entrada pelo seu peso correspondente e irá retornar o resultado +1 caso a entrada seja maior ou igual zero, ou -1 caso contrário. Esse sistema fornece um modelo computacional completo, podendo calcular qualquer função lógica.

Em 1958, Frank Rosenblatt [52] apresenta o perceptron, um algoritmo de treinamento para um tipo de rede com camada única. Esta invenção inspirou engenheiros, físicos e matemáticos a dedicar seus esforços de pesquisa para diferentes aspectos das redes neurais na década de 1960 e 1970 [20]. O perceptron é similar ao neurônio de McCulloch-Pitts. Os valores de entrada e níveis de ativação são +1 ou -1 e os pesos têm valores reais. O nível de ativação é dado pela soma dos valores ponderados de entrada $x_{i}, i=1, \ldots, n$, ou seja, $\sum_{i=1}^{n} w_{i} x_{i}$, onde $w_{1}$ é o peso de cada entrada. Usando um limiar (bias) $b$, a saída do neurônio será +1 , se $\sum_{i=1}^{n} w_{i} x_{i} \geq b$, ou -1 caso $\sum_{i=1}^{n} w_{i} x_{i}<b$. Isso representa uma forma simples de aprendizado. Após tentar resolver uma ocorrência de problema, um professor fornece a ele um resultado correto. Deste modo, o perceptron pode ajustar seu conjunto de pesos (caso exista) de modo a minimizar o erro médio sobre todo o conjunto de treinamento[35].

Já na década de 1980, o físico, biólogo e neurologista John Hopfield desenvolve uma arquitetura de rede neural com uma camada, com os neurônios dessa camada ligados a todos os outros, o que torna essa rede auto-associativa [23]. Usando o conceito de minimização de energia, Hopfield estuda as propriedades de convergência de uma família de redes que ele concebeu, as Redes Neurais de Hopfield, cuja dinâmica é baseada no comportamento das sinapses dos neurônios no cérebro humano. Possui diversas aplicações, como por exemplo, em neurociência, otimização e reconhecimento de padrões.

As Redes Neurais de Hopfield podem ser implementadas em hardware, combinando componentes como capacitores e resistores, um recurso bastante significativo. Em 2008, Boroomand e Menhaj [4], propuseram as Redes Neurais de Hopfield Fracionária (FHNN), que substitui os capacitores clássicos por um componente denominado Capacitor fracionário. O Capacitor fracionário é um capacitor generalizado, um circuito elétrico no qual sua tensão e corrente são relacionadas por uma equação diferencial ordinária de ordem fracionária (EDOF) 48, 44, 4].

As EDOFs têm como principal característica o uso de operadores que generalizam o conceito de derivada para ordem não-inteira, sendo conhecidas como derivadas fracionarias. Estas derivadas existem há mais de 300 anos e atualmente possuem muitas aplicações na física e engenharia, como na bioengenharia [38], controle [3] e viscoelasticidade [21], pois para descrever alguns sistemas o uso de derivadas fracionárias é mais acurado. Um exemplo foi a aplicação de Anastasio no circuito neural chamado de Integrador Oculomotor, uma rede que consiste de neurônios motores e pré-motores que mediam alguns subsistemas oculomotores, como os responsáveis pela perseguição e movimento rápido do olho entre os pontos de fixação, retransmitindo comandos de movimento ocular para motoneurônios extra-oculares. Anastásio [56] usa o cálculo fracionário para caracterizar a dinâmica em neurônios motores e pré-motores, onde ele aponta que o integrador oculomotor pode 
ter ordem fracionária, dependendo da velocidade do olho e dos comandos de posição dos olhos.

A principal motivação para o uso de derivadas fracionárias em modelos de redes neurais é que tais derivadas possuem melhor descrição de memória e propriedades hereditárias de vários processos que os de ordem inteira [61, 48]. Isso oferece uma vantagem com relação às derivadas de ordem inteira na modelagem de sistemas biológicos complexos, com comportamento não-linear e memória de longo prazo. Assim, temos uma melhoria importante com a incorporação de um termo de memória (na forma de uma derivada fracionária) em um modelo de rede neural como o de Hopfield. Em 2008, pesquisadores descobriram que o cálculo fracionário poderia ser bem usado no estudo das redes neurais, devido ao fato da diferenciação de ordem fracionada fornecer neurônios com um capacidade computacional fundamental, que contribui para o processamento eficiente da informação, antecipação do estímulo, e mudanças de fase independentes de frequência em disparos neuronais oscilatórios [36].

Para EDOFs, foi proposto em 2009 um critério especial de estabilidade, a estabilidade de MittagLeffler [32]. Esse critério é uma extensão do método direto de Lyapunov para analisar os pontos de equilíbrio de sistemas com derivadas em ordem fracionária. O objetivo deste trabalho é o estudo da estabilidade de Mittag-Leffler do ponto de equilíbrio de uma rede neural de Hopfield fracionária. Haykin [20] define o estudo da dinâmica das redes neurais, com especial ênfase na estabilidade, como neurodinâmica. A presença de estabilidade sempre implica alguma forma de coordenação entre as partes individuais do sistema [20]. Assim, para aplicações das redes neurais de Hopfield fracionária em computação paralela e processamento de sinais, é necessário que exista uma solução computável bem definida para todos os possíveis estados iniciais. Do ponto de vista matemático, a análise de estabilidade de um ponto de equilíbrio único é necessária e valiosa [61.

Este trabalho está organizado em quatro capítulos. No primeiro capítulo introduzimos as duas principais definições de derivadas fracionárias, a derivada de Riemann-Liouville e a derivada de Caputo, mostrando conceitos e propriedades que serão importantes no desenvolvimento do texto. Além disso, apresentamos uma interpretação física baseada no conceito de memória e algumas relações entre ambas as derivadas.

No capítulo 2 introduzimos a função de Mittag-Leffler, a transformada de Laplace e como essas ferramentas são importantes na resolução de uma EDOF. Também faremos um estudo para determinar critérios de existência e unicidade das soluções destas equações. Por último, apresentaremos a estabilidade de Lyapunov e Mittag-Leffler e dois critérios de estabilidade em EDOFs.

No Capítulo 3 fazemos uma apresentação das Redes Neurais de Hopfield e Hopfield fracionária contínuas e estudamos sua estabilidade através da análise da função de energia. Além disso, mostramos uma aplicação das RNHF para identificação dos parâmetros de um sistema e também na recuperação de dados corrompidos de uma imagem.

Já no capítulo 4, apresentamos um critério de estabilidade para RNH e também um critério para a estabilidade de Mittag-Leffler em RNH fracionárias. Alguns exemplos são resolvidos com o objetivo de verificar o resultado teórico.

As redes neurais fracionárias (RNHF) é um tópico aberto e significativo em teoria e aplicações [60]. Esperamos que com esse trabalho possamos contribuir para a divulgação do método de MittagLeffler para avaliar a estabilidade de EDOF's e também das redes neurais fracionárias, demonstrando sua aplicabilidade e importância. 


\section{Capítulo 2}

\section{Cálculo Fracionário}

Apresentaremos neste capítulo conceitos preliminares que serão fundamentais no decorrer deste trabalho, em particular a função gama, o conceito de integral e derivada de ordens fracionárias e propriedades úteis. Na literatura existem várias interpretações de derivadas e integrais fracionárias, como as definições de Riemann-Liouville, Caputo, Riesz, Hadamard e Grünwald-Letnikov. Neste trabalho, apenas as definições de Riemann-Liouville e Caputo serão utilizadas.

\subsection{Função Gama}

A função gama é uma das funções mais importantes da física-matemática. Foi primeiramente introduzida pelo matemático Leonhard Euler, com o objetivo de interpolar o fatorial sempre que o argumento da função é um inteiro. Além disso, pode ser utilizada no cálculo da transformada de Laplace e de algumas funções. Eis a sua definição:

Definição 2.1 (Função Gama) Seja z um número complexo. A função gama é uma função complexa definida como

$$
\Gamma(z)=\int_{0}^{\infty} t^{z-1} e^{-t} d t
$$

Esta integral converge na metade do plano complexo $\operatorname{Re}(z)>0$, pois

$$
\begin{aligned}
\Gamma(x+i y)= & \int_{0}^{\infty} e^{-t} t^{x+i y-1} d t=\int_{0}^{\infty} e^{-t} t^{x-1} e^{i y \log (t)} d t \\
& \int_{0}^{\infty} e^{-t} t^{x-1}[\cos (y \log (t))+i \operatorname{sen}(y \log (t))] d t
\end{aligned}
$$

e a convergência é garantida pelo termo $e^{-t}$. Para $t=0$, a função converge quando $x=\operatorname{Re}(z)>1$. Na Figura 2.1. no caso particular em que $z$ é real, podemos verificar sua representação gráfica.

Uma propriedade fundamental da função gama é que ela satisfaz a seguinte igualdade:

$$
\Gamma(z+1)=z \Gamma(z)
$$

que pode ser verificada fazendo integração por partes na integral em 2.1):

$$
\Gamma(z+1)=\int_{0}^{\infty} e^{-t} t^{z} d z=\left[-e^{-t} t^{z}\right]_{t=0}^{t=\infty}+z \int_{0}^{\infty} e^{-t} t^{z-1} d t=z \Gamma(z),
$$

que para $n \in \mathbb{N}$, podemos escrever

$$
\Gamma(n+1)=n !
$$

Outra função conhecida é a função beta, definida como

$$
B(x, y)=\int_{0}^{\infty} \frac{t^{x-1}}{(1+t)^{x+y}} d t
$$




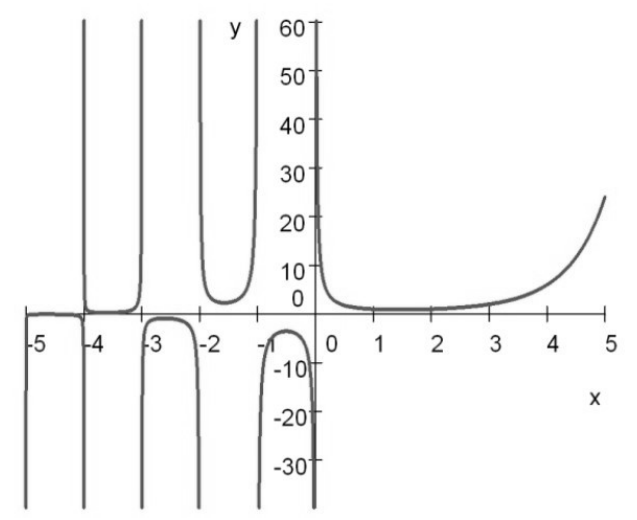

Figura 2.1: Função gama no intervalo $[-5,5]$

onde $\operatorname{Re}(x), \operatorname{Re}(y)>0$. Fazendo $s=t /(1+t)$, temos

$$
B(x, y)=\int_{0}^{1} s^{x-1}(1-s)^{y-1} d s, \operatorname{com} \operatorname{Re}(x), \operatorname{Re}(y)>0 .
$$

O seguinte teorema estabelece uma relação entre as funções gama e beta:

Teorema 2.1 Para as variáveis complexas $x$ e y $\operatorname{com} \operatorname{Re}(x)>0$ e $\operatorname{Re}(y)>0$,

$$
B(x, y)=\frac{\Gamma(x) \Gamma(y)}{\Gamma(x+y)} .
$$

Demonstração. Da Equação 2.1, temos

$$
\Gamma(x) \Gamma(y)=\int_{0}^{\infty} e^{-t} t^{x-1} d t \int_{0}^{\infty} e^{-s} s^{y-1} d s=\int_{0}^{\infty} \int_{0}^{\infty} e^{-(t+s)} t^{x-1} s^{y-1} d t d s .
$$

Introduzindo a mudança de variável $t=u v$ e $s=u(1-v)$, temos que $t+s=u$ e $d t d s=u d u d v$. Portanto

$$
\begin{aligned}
\Gamma(x) \Gamma(y) & =\int_{0}^{1} \int_{0}^{\infty} e^{-u} u^{x-1} y^{x-1} u^{y-1}(1-v)^{y-1} u d u d v \\
& =\int_{0}^{\infty} e^{-u} u^{x+y+1} d u \int_{0}^{1} v^{x-1}(1-v)^{y-1} d v=\Gamma(x+y) B(x, y),
\end{aligned}
$$

que leva à Equação (2.3).

Para mais detalhes a respeito de propriedades das funções gama, beta e de outras funções especias, o livro [1] é uma ótima referência de leitura.

\section{$2.2 \quad$ Integral e derivada de Riemann-Liouville}

Vamos primeiramente introduzir uma motivação para a definição da integral de RiemannLiouville. Seja $f: I \rightarrow \mathbb{R}$ uma função contínua por partes no intervalo $[0, \infty]$ e integrável em todo o subintervalo $[0, \infty)$ e $J^{n} f(t)=(J J \ldots J) f(t)$ a composição $n$ vezes do operador integral

$$
J f(t)=\int_{0}^{t} f(\tau) d \tau
$$

Observe que

$$
J^{2} f(t)=J J f(t)=\int_{0}^{t} J f\left(\tau_{1}\right) d \tau_{1}=\int_{0}^{t} \int_{0}^{\tau_{1}} f(\tau) d \tau d \tau_{1}
$$


Do teorema de Fubini, temos:

$$
\begin{aligned}
J^{2} f(t) & =\int_{0}^{t} \int_{0}^{\tau_{1}} f(\tau) d \tau d \tau_{1}=\int_{0}^{t} \int_{\tau}^{t} f(\tau) d \tau_{1} d \tau=\int_{0}^{t} f(\tau)(t-\tau) d \tau \\
J^{3} f f(t) & =J J^{2} f(t)=\int_{0}^{t} J^{2} f\left(\tau_{1}\right) d \tau_{1}=\int_{0}^{t} \int_{0}^{\tau_{1}} f(\tau)\left(\tau_{1}-\tau\right) d \tau d \tau_{1} \\
& =\int_{0}^{t} \int_{\tau}^{t} f(\tau)\left(\tau_{1}-\tau\right) d \tau_{1} s \tau=\int_{0}^{t} f(\tau) \frac{(t-\tau)^{2}}{2} d \tau
\end{aligned}
$$

Fazendo a composição do operador $J f(t) n$ vezes obtemos a expressão a seguir, conhecida como fórmula de Cauchy:

$$
\begin{gathered}
J^{n} f(t):=\int_{0}^{t} \int_{0}^{\tau_{n-1}} \ldots \int_{0}^{\tau_{1}} f(\tau) d(\tau) d\left(\tau_{1}\right) \ldots d\left(\tau_{n-1}\right), \\
J^{n} f(t)=\frac{1}{(n-1) !} \int_{0}^{t} f(\tau)(t-\tau)^{(n-1)} d \tau .
\end{gathered}
$$

Usando a função gama, temos que o lado direito da expressão (2.4) pode fazer sentido com $n \in \Re$. Assim, é natural definirmos a integral fracionária como:

Definição 2.2 (Integral de Riemann-Liouville) Sejam $\alpha>0, f(x)$ contínua por partes no intervalo $[0, \infty)$ e integrável em um subintervalo $[a, b]$. Então para $x \in[a, b]$ definimos a integral fracionária de Riemann-Liouville de ordem a como sendo a seguinte integral

$$
{ }_{a} \mathrm{~J}_{x}^{\alpha} f(x)=\frac{1}{\Gamma(\alpha)} \int_{a}^{x} \frac{f(\tau)}{(x-\tau)^{1-\alpha}} d \tau
$$

Mostraremos agora uma propriedade muito importante para a integral fracionária, conhecida como lei dos expoentes.

Teorema 2.2 (Lei dos Expoentes) [48] Sejam $\alpha, \beta>0$ e $f(x)$ contínua por partes em $(0, \infty)$. Temos que a integral fracionária de Riemann-Liouville satisfaz a relação

$$
{ }_{a} \mathrm{~J}_{x}^{\alpha}\left({ }_{a} \mathrm{~J}_{x}^{\beta} f(x)\right)={ }_{a} J_{x}^{\alpha+\beta} f(x)
$$

Demonstração. Temos de fato que

$$
\begin{aligned}
{ }_{a} \mathrm{~J}_{x}^{\alpha}\left({ }_{a} \mathrm{~J}_{x}^{\beta} f(x)\right) & =\frac{1}{\Gamma(\beta)} \int_{a}^{x}(x-\tau)^{\beta-1}{ }_{a} \mathrm{~J}_{\tau}^{\alpha} f(\tau) d \tau \\
& =\frac{1}{\Gamma(\alpha) \Gamma(\beta)} \int_{a}^{x}(x-\tau)^{\beta-1} d \tau \int_{a}^{\tau}\left(\tau-\tau_{1}\right)^{\alpha-1} f\left(\tau_{1}\right) d \tau_{1} .
\end{aligned}
$$

Usando o teorema de Fubini para mudar a ordem de integração e tomando $\tau=\tau_{1}+s\left(x-\tau_{1}\right)$, temos:

$$
\begin{aligned}
{ }_{a} \mathrm{~J}_{x}^{\alpha}\left({ }_{a} \mathrm{~J}_{x}^{\beta} f(x)\right) & =\frac{1}{\Gamma(\alpha) \Gamma(\beta)} \int_{a}^{x} f\left(\tau_{1}\right) \int_{\tau_{1}}^{\tau}(x-\tau)^{\beta-1}\left(\tau-\tau_{1}\right)^{\alpha-1} d \tau d \tau_{1} \\
& =\frac{B(\alpha, \beta)}{\Gamma(\alpha) \Gamma(\beta)} \int_{a}^{x}\left(x-\tau_{1}\right)^{\alpha+\beta-1} f\left(\tau_{1}\right) d \tau_{1} \\
& ={ }_{a} \mathrm{~J}^{\alpha+\beta} f(x) .
\end{aligned}
$$

A Equação (2.5) é uma integral imprópria quando $0<\alpha<1$. A hipótese de que $f$ é contínua por partes em $(0, \infty)$ é tomada com o intuito de que funções com comportamento similar a $\log (t)$ e $t^{n}, \mathrm{com}-1<n<0$ em uma vizinhança da origem também tenham sua integral fracionária bem definida [11]. 
Exemplo 2.1 Vamos calcular a integral de Riemann-Liouville de ordem $\alpha$ para a função $x^{k}, k>$ -1: Introduzindo a mudança de variável $u=\frac{s}{x}$, temos:

$$
\begin{aligned}
{ }_{0} \mathrm{~J}_{x}^{\alpha} f(x) & =\frac{1}{\Gamma(\alpha)} \int_{0}^{x} \frac{f(s)}{(x-s)^{1-\alpha}} d s \\
{ }_{0} \mathrm{~J}_{x}^{\alpha} x^{k} & =\frac{1}{\Gamma(\alpha)} \int_{0}^{x} x^{\alpha-1}\left(1-\frac{s}{x}\right)^{\alpha-1} s^{k} d s \\
& =\frac{1}{\Gamma(\alpha)} \int_{0}^{1} x^{\alpha-1}(1-u)^{\alpha-1}(x u)^{k} x d u \\
& =\frac{1}{\Gamma(\alpha)} \int_{0}^{1} x^{\alpha}(1-u)^{\alpha-1} x^{k} u^{k} d u \\
& =\frac{x^{\alpha+k}}{\Gamma(\alpha)} \int_{0}^{1}(1-u)^{\alpha-1} u^{k} d u
\end{aligned}
$$

Usando a função beta em (2.6), temos

$$
{ }_{0} \mathrm{~J}_{x}^{\alpha} x^{k}=\frac{x^{\alpha+k}}{\Gamma(\alpha)} B(k+1, \alpha)=\frac{\Gamma(k+1)}{\Gamma(k+\alpha+1)} x^{\alpha+k}
$$

\section{Derivada de ordem fracionária}

A definição da derivada de ordem fracionária, segundo Riemann-Liouville, é baseada essencialmente no fato da derivada ser a operação inversa da integração na lei dos expoentes, e estabelece que a derivada de ordem fracionária é a derivada de ordem inteira de uma determinada integral de ordem fracionária.

Definição 2.3 (Derivada de Riemann-Liouville) Sejam $\alpha>0$ e $n$ o menor inteiro maior que a. A derivada de Riemann-Liouville da função $f$ é

$$
{ }_{a}^{\mathrm{RL}} \mathrm{D}_{x}^{\alpha} f(x)=D^{n}\left[{ }_{a} \mathrm{~J}_{x}^{n-\alpha} f(x)\right]=\frac{1}{\Gamma(n-\alpha)} \frac{d^{n}}{d x^{n}} \int_{a}^{x} \frac{f(\tau) d \tau}{(x-\tau)^{\alpha-n+1}}
$$

na qual $D^{n}$ é a derivada de ordem inteira $e_{a} \mathrm{~J}^{n-\alpha}$ a integral de Riemann-Liouville

Exemplo 2.2 Usando a definição (2.3), vamos calcular a derivada de Riemann-Liouville de ordem $\alpha$ de $f(x)=x^{k}$, com $k>-1$. Usando a Equação 2.7), podemos escrever

$$
{ }_{0}^{\mathrm{RL}} \mathrm{D}_{x}^{\alpha} f(x)=D^{n}\left[{ }_{0} \mathrm{~J}_{x}^{n-\alpha} x^{k}\right]=\left[\frac{D^{n}\left(x^{n-\alpha+k}\right) \Gamma(k+1)}{\Gamma(k+n-\alpha+1)}\right] .
$$

Note que

$$
\begin{aligned}
D^{n}\left(x^{n-\alpha+k}\right)= & (n-\alpha+k)(n-\alpha+k-1)(n-\alpha+k-2) \ldots(n-\alpha+k-(n-1)) x^{n-\alpha+k-n} \\
= & \frac{(n-\alpha+k) !}{(-\alpha+k) !} x^{-\alpha+k}=\frac{\Gamma(n-\alpha+k+1)}{\Gamma(-\alpha+k+1)} x^{-\alpha+k} .
\end{aligned}
$$

Substituindo em (2.9), vamos ter

$$
{ }_{0}^{\mathrm{RL}} \mathrm{D}_{x}^{\alpha} x^{k}=\frac{x^{k-\alpha} \Gamma(k+1)}{\Gamma(k-\alpha+1)} .
$$

Veja na Equação 2.10, considerando $k=0$, que ${ }_{0}^{\mathrm{RL}} \mathrm{D}_{x}^{\alpha} x^{0}=\frac{x^{-\alpha}}{\Gamma(1-\alpha)}$, ou seja, a derivada de Riemann-Liouville de $f(x)=1$ não é zero. 
O teorema seguinte, cuja demonstração foi baseada em [55], nos mostra que a derivada de ordem $\alpha$ é inversa à esquerda, mas não necessariamente inversa à direita de uma integral de ordem $\alpha$. Para simplificar a notação, vamos supor aqui que ${ }_{a}^{\mathrm{RL}} \mathrm{D}_{x}^{\alpha}={ }^{\mathrm{RL}} \mathrm{D}_{x}^{\alpha}$ e ${ }_{a} \mathrm{~J}_{x}^{\alpha}=J_{x}^{\alpha}, a \geq 0$.

Teorema 2.3 Sejam $\alpha>0$ e $f$ uma função integrável no intervalo $[a, b]$, então valem as seguintes igualdades

$$
\begin{gathered}
{ }^{\mathrm{RL}} \mathrm{D}_{x}^{\alpha} J_{x}^{\alpha} f(x)=f(x) \\
J_{x}^{\alpha \mathrm{RL}} \mathrm{D}_{x}^{\alpha} f(x)=f(x)-\left.\sum_{k=1}^{n} \frac{1}{\Gamma(\alpha-k+1)} x^{\alpha-k} D^{n-k} J^{n-\alpha} f(x)\right|_{x=a}
\end{gathered}
$$

são válidos em todo intervalo $[a, b]$.

Demonstração. Para a Equação 2.11, devido a lei dos expoentes,temos

$$
{ }^{\mathrm{RL}} \mathrm{D}_{x}^{\alpha} J_{x}^{\alpha} f(x)=D^{m} J_{x}^{m-\alpha} J_{x}^{\alpha} f(x)=D^{m} J_{x}^{m-\alpha}=f(x)
$$

Na Equação 2.12, temos

$$
J_{x}^{\alpha \mathrm{RL}} \mathrm{D}_{x}^{\alpha} f(x)=D^{1} J_{x}^{\alpha+1 \mathrm{RL}} \mathrm{D}_{x}^{\alpha} f(x)=\frac{d}{d x}\left[\frac{1}{\Gamma(\alpha+1)} \int_{0}^{x}(x-s)^{\alpha}\left({ }^{\mathrm{RL}} \mathrm{D}_{s}^{\alpha} f(s)\right) d s\right]
$$

Usando integração por partes sucessivas vezes na integral da Equação (2.13), vamos obter

$$
\begin{aligned}
& \frac{1}{\Gamma(\alpha+1)} \int_{a}^{x}(x-s)^{\alpha}\left({ }^{\mathrm{RL}} \mathrm{D}^{\alpha} f(s)\right) d s \\
& \left.=\frac{1}{\Gamma(\alpha+1)} \int_{a}^{x}(x-s)^{\alpha} D^{m} J_{s}^{m-\alpha} f(s)\right) d s \\
& =\frac{1}{\Gamma(\alpha+1)}\left(\left.(x-s)^{\alpha} D^{m-1} J_{s}^{m-\alpha} f(s)\right|_{a} ^{x}-\frac{1}{\Gamma(\alpha)} \int_{a}^{x}(x-s)^{\alpha-1} D^{m-1} J_{s}^{m-\alpha} f(s) d s\right) \\
& =-\left.\frac{1}{\Gamma(\alpha+1)} x^{\alpha} D^{m-1} J_{x}^{m-\alpha} f(x)\right|_{x=a}-\frac{1}{\Gamma(\alpha)} \int_{a}^{x}(x-s)^{\alpha-1} D^{m-1} J_{s}^{m-\alpha} f(s) d s \\
& =-\left.\frac{1}{\Gamma(\alpha+1)} x^{\alpha} D^{m-1} J_{x}^{m-\alpha} f(x)\right|_{x=a}- \\
& -\frac{1}{\Gamma(\alpha}\left(\left.(x-s)^{\alpha-1} D^{m-2} J_{s}^{m-\alpha} f(s)\right|_{a} ^{x}-(\alpha-1) \int_{a}^{x}(x-s)^{\alpha-2} D^{m-2} J_{s}^{m-\alpha} f(s) d s\right) \\
& =-\left.\frac{1}{\Gamma(\alpha+1)} x^{\alpha} D^{m-1} J_{x}^{m-\alpha} f(x)\right|_{x=a} \\
& -\left.\frac{1}{\Gamma(\alpha)} x^{\alpha-1} D^{m-2} J_{x}^{m-\alpha} f(x)\right|_{x=a}-\frac{1}{\Gamma(\alpha-2)} \int_{a}^{x}(x-s)^{\alpha-2} D^{m-2} J_{s}^{m-\alpha} f(s) d s \\
& \vdots \\
& =-\left.\frac{1}{\Gamma(\alpha+1)} x^{\alpha} D^{m-1} J_{x}^{m-\alpha} f(x)\right|_{x=a}-\left.\frac{1}{\Gamma(\alpha)} x^{\alpha-1} D^{m-2} J_{x}^{m-\alpha} f(x)\right|_{x=a}-\ldots \\
& \ldots-\left.\frac{1}{\Gamma(\alpha+1-(m-1))} x^{\alpha-m+1} J_{x}^{m-\alpha} f(x)\right|_{x=a}-\frac{1}{\Gamma(\alpha-m+1)} \int_{a}^{x}(x-s)^{\alpha-m} J_{s}^{m-\alpha} f(s) d s \\
& =-\left.\sum_{k=1}^{m} \frac{1}{\Gamma(\alpha+2-k)} x^{\alpha-k+1} D^{m-k} J^{m-\alpha} f(x)\right|_{x=a}+J_{x}^{\alpha-m+1} J_{x}^{m-\alpha} f(x) \\
& =-\left.\sum_{k=1}^{m} \frac{1}{\Gamma(\alpha+2-k)} x^{\alpha-k+1} D^{m-k} J^{m-\alpha} f(x)\right|_{x=a}+J_{x}^{1} f(x) \\
& =-1
\end{aligned}
$$


o que implica em

$$
\begin{aligned}
J_{x}^{\alpha} D^{\alpha} f(x) & =\frac{d}{d x}\left(-\left.\sum_{k=1}^{m} \frac{1}{\Gamma(\alpha-k+2)} x^{\alpha-k+1} D^{m-k} J^{m-\alpha} f(x)\right|_{x=a}+J_{x}^{1} f(x)\right) \\
& =f(x)-\left.\sum_{k=1}^{m} \frac{\alpha-k+1}{(\alpha-k+1) \Gamma(\alpha-k+1)} x^{\alpha-k} D^{m-k} J_{x}^{m-\alpha} f(x)\right|_{x=a} \\
& =f(x)-\left.\sum_{k=1}^{m} \frac{1}{\Gamma(\alpha-k+1)} x^{\alpha-k} D^{m-k} J_{x}^{m-\alpha} f(x)\right|_{x=a} .
\end{aligned}
$$

\subsection{Derivada de Caputo}

Vimos na seção anterior que a derivada fracionária de Riemann-Liouville é a derivada de ordem inteira de uma integral fracionária. Em 1969, M. Caputo [8] desenvolveu uma nova definição para derivada fracionária com o objetivo de resolver alguns problemas relacionados à viscoelasticidade. Vamos agora apresentar a definição de derivada fracionária segundo Caputo [7, muito similar à derivada de Riemann-Liouville, porém a ordem de integração fracionária com a derivada é invertida.

Definição 2.4 Sejam $\alpha>0, n \in \mathbb{N}$ o menor inteiro maior que $\alpha$ e $f(t)$ uma função integrável de ordem n. A derivada de Caputo da função $f$ é

$$
\begin{gathered}
{ }_{a}^{\mathrm{C}} \mathrm{D}_{x}^{\alpha} f(x)={ }_{a} \mathrm{~J}_{x}^{n-\alpha} D^{n} f(x) \\
{ }_{a}^{\mathrm{C}} \mathrm{D}_{x}^{\alpha} f(x)=\left\{\begin{array}{l}
\frac{1}{\Gamma(n-\alpha)} \int_{0}^{x} \frac{f^{(n)}(\tau)}{(x-\tau)^{\alpha+1-n}} d \tau, \text { se } n-1<\alpha<n \\
\frac{d^{n}}{d x^{n}} f(x), \text { se } \alpha=n
\end{array}\right.
\end{gathered}
$$

De fato, quando $\alpha \rightarrow n$, a derivada de Caputo da função $f(x)$ torna-se a n-ésima derivada da função $f(x)$. Para mostrar isto. vamos admitir que $0 \leq n-1<\alpha<n$ e que a função $f(x)$ tem derivada de ordem $n+1$ no intervalo $[a, b]$ para $b>a$. Integrando por partes temos

$$
\begin{aligned}
{ }_{a}^{\mathrm{C}} \mathrm{D}_{x}^{\alpha} f(x) & =\frac{1}{\Gamma(n-\alpha)} \int_{a}^{x} \frac{f^{(n)}(\tau)}{(x-\tau)^{\alpha+1-n}} d \tau \\
& =\frac{1}{\Gamma(n-\alpha)}\left(-\left.f^{(n)}(\tau) \frac{(x-\tau)^{n-\alpha}}{n-\alpha}\right|_{a} ^{x}-\int_{a}^{x}-f^{(n+1)}(\tau) \frac{(x-\tau)^{n-\alpha}}{n-\alpha} d \tau\right) \\
& =\frac{1}{\Gamma(n-\alpha+1)}\left(f^{(n)}(a) x^{n-a}+\int_{a}^{x} f^{(n+1)}(\tau)(x-\tau)^{n-\alpha} d \tau\right) .
\end{aligned}
$$

Tomando o limite para $\alpha \rightarrow n$,

$$
\lim _{\alpha \rightarrow n}{ }_{a} \mathrm{D}_{x}^{\alpha} f(x)=f^{(n)}(a)+\left.f^{(n)}(\tau)\right|_{a} ^{x}=f^{(n)}(x) .
$$

A definição (2.4) é mais restritiva que a definição de Riemann-Liouville uma vez que requer a integrabilidade da derivada de ordem $n$ da função.

Exemplo 2.3 Vamos calcular a derivada de ordem $\alpha$, segundo Caputo, da função $f(x)=x^{\mu}$ com $\mu \in(-1,0]$. No caso em que $\mu$ é um número natural temos, para $\mu>n$

$$
D^{n} x^{\mu}=\frac{\mu !}{(\mu-n) !} x^{\mu-n} .
$$


Usando a função gama, podemos escrever a expressão acima como

$$
D^{n} x^{\mu}=\frac{\Gamma(\mu+1)}{\Gamma(\mu-n+1)} x^{\mu-n} .
$$

Do exemplo 2.2, temos que

$$
J^{\alpha} x^{\mu-n}=\frac{\Gamma(\mu-n+1)}{\Gamma(\mu-n+\alpha+1)} x^{\mu-n-\alpha} .
$$

A partir disto podemos escrever

$$
\begin{gathered}
{ }^{C} D_{x}^{\alpha} x^{\mu}=J^{n-\alpha}\left[D^{n} x^{n}\right]=J^{n-\alpha}\left[\frac{\Gamma(\mu+1)}{\Gamma(\mu-n+1)} x^{\mu-n}\right] \\
=\left[\frac{\Gamma(\mu+1)}{\Gamma(\mu-\alpha+1)} x^{\mu-\alpha}\right] .
\end{gathered}
$$

\subsection{Derivada de Caputo vs Riemann-Liouville}

A primeira diferença que devemos notar é que se $f(x)$ é uma função na qual as integrais e derivadas fracionárias podem ser aplicadas temos que

$$
{ }_{a}^{\mathrm{RL}} \mathrm{D}_{x}^{\alpha} f(x)=D^{m}\left[{ }_{a} \mathrm{~J}^{m-\alpha} f(x)\right] \neq{ }_{a} \mathrm{~J}^{m-\alpha}\left[D^{m} f(x)\right]={ }_{a}^{\mathrm{C}} \mathrm{D}_{x}^{\alpha} f(x),
$$

ou seja, de maneira geral, ao comutar a integral fracionária com a derivada, alteramos o resultado final.

Vale ressaltar que se $f(x)$ e suas derivadas de ordem inteira menores que $m$ se anulam em $x=0+$, então a comutação dos operadores é verdadeira. De fato, para que possamos comutar a integral com a derivada é suficiente que em ambos os casos a integral convirja uniformemente. É por esta razão que a derivada fracionária de ordem $\alpha>0$ da função $f(x)=x^{\mu}, \mu \in(-1,0]$ coincide quando utilizamos a versão de Riemann-Liouville e a versão de Caputo, pois $f^{(n)}\left(0^{+}\right)=0$ para todo $n \leq m-1$. 11 .

Embora para polinômios não constantes as definições segundo Riemann-Liouville e Caputo para a derivada fracionária coincidam, isto não ocorre para constantes, uma vez que na definição de Caputo, primeiro tomamos a derivada de ordem inteira e depois a integral fracionária. A derivada de ordem fracionária segundo Caputo de uma constante é nula, ou seja, ${ }^{C} \mathrm{D}_{x}^{\alpha} 1=0$, o que não ocorre quando calculamos a derivada segundo Riemann-Liouville. Partindo da interpretação física deste fato, os autores [12] e [48] consideram a derivada segundo Caputo mais convenientes para aplicações que a derivada segundo Riemann-Liouville.

\section{Outras propriedades das derivadas fracionárias}

Há ainda outras propriedades a respeito das derivadas fracionárias que devem ser mencionadas. Vamos considerar nesta seção as mais importantes para a derivada de Riemann-Liouville e Caputo.

\section{Linearidade}

Sejam $f_{1}(x)$ e $f_{2}(x)$ duas funções no intervalo $[a, b]$ e $c_{1}, c_{2} \in \mathbb{R}$. As seguintes equações são asseguradas,

$$
\begin{aligned}
{ }_{a}^{\mathrm{RL}} \mathrm{D}_{x}^{\alpha}\left(f_{1}(x)+f_{2}(x)\right) & ={ }_{a}^{\mathrm{RL}} \mathrm{D}_{x}^{\alpha} f_{1}(x)+{ }_{a}^{\mathrm{RL}} \mathrm{D}_{x}^{\alpha}\left(f_{2}(x)\right),{ }_{a}^{\mathrm{RL}} \mathrm{D}_{x}^{\alpha}\left(c_{1} f_{1}(x)\right)=c_{1}{ }_{a}^{\mathrm{RL}} \mathrm{D}_{x}^{\alpha}\left(f_{1}(x)\right) \\
{ }_{a}^{\mathrm{C}} \mathrm{D}_{x}^{\alpha}\left(f_{1}(x)+f_{2}(x)\right) & ={ }_{a}^{\mathrm{C}} \mathrm{D}_{x}^{\alpha} f_{1}(x)+{ }_{a}^{\mathrm{C}} \mathrm{D}_{x}^{\alpha}\left(f_{2}(x),{ }_{a}^{\mathrm{C}} \mathrm{D}_{x}^{\alpha}\left(c_{1} f_{1}(x)\right)=c_{1}{ }_{a}^{\mathrm{C}} \mathrm{D}_{x}^{\alpha}\left(f_{1}(x)\right),\right.
\end{aligned}
$$

que podem ser demonstradas diretamente das propriedades de linearidade da integral. 


\section{Inversa à direita da Derivada de Caputo}

Como sabemos, a derivada de Caputo é inversa à esquerda da integral de Riemann-Liouville, ou seja,

$$
{ }_{a}^{\mathrm{C}} \mathrm{D}_{x}^{\alpha}\left({ }_{a} \mathrm{~J}^{\alpha} f(x)\right)=f(x) .
$$

Porém, de forma análoga à derivada de Riemann-Liouville (Teorema 2.3), a inversa à direita da derivada de Caputo é dada pela expressão:

$$
{ }_{a} \mathrm{~J}^{\alpha}\left({ }_{a}^{\mathrm{C}} \mathrm{D}_{x}^{\alpha} f(x)\right)=f(x)-\sum_{k=0}^{m-1} \frac{D^{k} f(a)}{k !}(x-a)^{k} .
$$

Demonstração. Da definição da derivada de Caputo e aplicando a integral de Riemann-Liouville em ambos os lados, temos

$$
{ }_{a} \mathrm{~J}_{x}^{\alpha}\left({ }_{a}^{\mathrm{C}} \mathrm{D}_{x}^{\alpha} f(x)\right)={ }_{a} \mathrm{~J}_{x}^{\alpha}\left({ }_{a} \mathrm{~J}_{x}^{n-\alpha} D^{n} f(x)\right)=J_{x}^{n} D^{n} f(x) .
$$

Usando a expansão de Taylor em $f(x)$, vamos obter

$$
f(x)=\sum_{k=0}^{n} \frac{(x-a)^{k} f^{(k)}(a)}{k !}+R_{n}
$$

onde $R_{n}$ é o resto de Lagrange, equivalente a

$$
R_{n}=\int_{a}^{x} f^{(n+1)}(\tau) \frac{(x-\tau)^{n}}{n !} d \tau={ }_{a} \mathrm{~J}_{x}^{n} D^{n} f(x)
$$

Substituindo 2.21 em 2.20, obtemos

$$
{ }_{a} \mathrm{~J}^{\alpha}\left({ }_{a}^{\mathrm{C}} \mathrm{D}_{x}^{\alpha} f(x)\right)=f(x)-\sum_{k=0}^{m-1} \frac{D^{k} f(a)}{k !}(x-a)^{k} .
$$

\section{Relação entre as derivadas de Caputo e Riemann-Liouville}

O seguinte teorema relaciona as derivadas de Riemann-Liouville e Caputo:

Teorema 2.4 [16] Seja $x>0, \alpha \in \mathbb{R}, n-1<\alpha<n$ e $n \in \mathbb{N}$. Então a seguinte relação entre as derivadas de Riemann-Liouville e Caputo é assegurada:

$$
{ }_{a}^{\mathrm{C}} \mathrm{D}_{x}^{\alpha} f(x)={ }_{a}^{\mathrm{RL}} \mathrm{D}_{x}^{\alpha} f(x)-\sum_{j=0}^{n-1} \frac{x^{k-\alpha}}{\Gamma(k+1-\alpha)} f^{(k)}(0)
$$

Demonstração. Da derivada de Riemann-Liouville da função potência Equação $(2.10)$, linearidade da derivada facionária e usando a expansão em série de Taylor com resto de Lagrange de $f(x)$, 
temos

$$
\begin{aligned}
{ }_{a}^{\mathrm{RL}} \mathrm{D}_{x}^{\alpha} f(x) & ={ }_{a}^{\mathrm{RL}} \mathrm{D}_{x}^{\alpha}\left(\sum_{k=0}^{n-1} \frac{x^{k}}{\Gamma(k+1)} f^{(k)}(0)+R_{n}\right) \\
& =\sum_{k=0}^{n-1} \frac{a}{\Gamma(k+1)} f^{\alpha} x^{k}(0)+{ }_{a}^{\mathrm{RL}} \mathrm{D}^{\alpha} R_{n} \\
& =\sum_{k=0}^{n-1} \frac{\Gamma(k+1) x^{k-\alpha}}{\Gamma(k-\alpha+1) \Gamma(k+1)} f^{(k)}(0)+{ }_{a}^{\mathrm{RL}} \mathrm{D}_{x}^{\alpha} J_{x}^{n} f^{(n)}(x) \\
& =\sum_{k=0}^{n-1} \frac{x^{k-\alpha}}{\Gamma(k-\alpha+1)} f^{(k)}(0)+J_{x}^{n-\alpha} D^{n} f(x) \\
& =\sum_{k=0}^{n-1} \frac{x^{k-\alpha}}{\Gamma(k-\alpha+1)} x^{(k)}(0)+{ }_{a}^{\mathrm{C}} \mathrm{D}_{x}^{\alpha} f(x),
\end{aligned}
$$

o que leva à Equação 2.22 .

\section{Não Comutatividade}

No geral, a lei dos expoentes não é válida para as derivadas de ordem fracionária. Seja $n-1<$ $\alpha<n$. Para a derivada de Riemann-Liouville temos

$$
{ }_{a}^{\mathrm{RL}} \mathrm{D}_{x}^{m}\left({ }_{a}^{\mathrm{RL}} \mathrm{D}_{x}^{\alpha} f(x)\right)={ }_{a}^{\mathrm{RL}} \mathrm{D}_{x}^{\alpha+m} f(x) \neq{ }_{a}^{\mathrm{RL}} \mathrm{D}_{x}^{\alpha}\left({ }_{a}^{\mathrm{RL}} \mathrm{D}_{x}^{m} f(x)\right), m, n \in \mathbb{N}, \alpha \in \mathbb{R},
$$

o que é diferente da derivada de Caputo, pois

$$
{ }_{a}^{\mathrm{C}} \mathrm{D}_{x}^{\alpha}\left({ }_{a}^{\mathrm{C}} \mathrm{D}_{x}^{m} f(x)\right)={ }_{a}^{\mathrm{C}} \mathrm{D}_{x}^{\alpha+m} f(x) \neq{ }_{a}^{\mathrm{C}} \mathrm{D}_{x}^{m}\left({ }_{a}^{\mathrm{C}} \mathrm{D}_{x}^{\alpha} f(x)\right), m, n \in \mathbb{N}, \alpha \in \mathbb{R} .
$$

As desigualdades 2.23 e 2.24 se tornam igualdade sob as seguintes condições adicionais [48]:

$$
\begin{aligned}
& f^{(s)}(0)=0, s=n, n+1, \ldots, m, \text { para }{ }_{a}^{\mathrm{C}} \mathrm{D}_{x}^{\alpha}, \\
& f^{(s)}(0)=0, s=0,1, . ., m, \text { para }{ }_{a}^{\mathrm{RL}} \mathrm{D}_{x}^{\alpha} .
\end{aligned}
$$

\section{Regra de Leibniz}

A regra de Leibniz tem como objetivo calcular a $n$-ésima derivada do produto de duas funções $f$ e $g$ contínuas, cuja fórmula é dada por

$$
D^{n}[f(x) g(x)]=\sum_{k=0}^{n}\left(\begin{array}{l}
n \\
k
\end{array}\right)\left[D^{k} g(x) D^{n-k} f(x)\right]
$$

onde $n \in \mathbb{N}$. Baseado em [12], [11] e [53, vamos agora estender este resultado para o cálculo em ordem fracionária.

Lema 2.1 Seja $\alpha>0$ e admita que a sequência $\left(f_{k}\right)_{k=1}^{\infty}$ é uma sequência uniformemente convergente de funções contínuas no intervalo $[a, b]$. Então podemos intercambiar o operador da integral fracionária com o limite da função, isto é

$$
{ }_{a} \mathrm{~J}_{x}^{\alpha} \lim _{k \rightarrow \infty} f_{k}(x)=\lim _{k \rightarrow \infty}{ }_{a} \mathrm{~J}_{x}^{\alpha} f_{k}(x)
$$

Em particular, a sequência $\left\{{ }_{a} \mathrm{~J}_{x}^{\alpha} f_{k}\right\}_{k=1}^{\infty}$ é uniformemente convergente. 
Demonstração. Seja $f$ o limite da sequência $\left(f_{k}\right)$. Sendo $f$ contínua, temos que

$$
\begin{aligned}
\left|{ }_{a} \mathrm{~J}_{x}^{\alpha} f_{k}(x)-{ }_{a} \mathrm{~J}_{x}^{\alpha} f(x)\right| & \leq \frac{1}{\Gamma(\alpha)} \int_{a}^{x}\left|f_{k}(t)-f(t)\right|(x-t)^{\alpha-1} d t \\
& \leq \frac{1}{\Gamma(\alpha)}\left\|f_{k}-f\right\|_{\infty} \int_{0}^{x}(x-t)^{\alpha-1} \\
& =\frac{1}{\Gamma(\alpha+1)}\left\|f_{k}-f\right\|_{\infty}(x-a)^{\alpha} \\
& =\frac{1}{\Gamma(\alpha+1)}\left\|f_{k}-f\right\|_{\infty}(b-a)^{n}
\end{aligned}
$$

que converge para zero quando $k \rightarrow \infty$ uniformemente para todo $x \in[a, b]$.

Lema 2.2 Seja $f(x)$ uma função analítica no intervalo $[a, b]$. Então a integral fracionária satisfaz

$$
{ }_{a} J_{x}^{\alpha} f(x)=\sum_{k=0}^{\infty}\left(\begin{array}{c}
-\alpha \\
k
\end{array}\right) \frac{f^{(k)}(x)}{\Gamma(k+\alpha+1)}(x-a)^{k+\alpha} .
$$

Demonstração. Temos que, para $\alpha>0$

$$
{ }_{a} \mathrm{~J}_{x}^{\alpha} f(x)=\frac{1}{\Gamma(\alpha)} \int_{a}^{x}(x-\tau)^{\alpha-1} f(\tau) d \tau .
$$

Utilizando a expansão de Taylor na função $f(\tau)$ em torno de $x$, temos

$$
{ }_{a} \mathrm{~J}_{x}^{\alpha} f(x)=\frac{1}{\Gamma(\alpha)} \int_{a}^{x}(x-\tau)^{\alpha-1} \sum_{k=0}^{\infty} \frac{(-1)^{k} f^{(k)}(x)}{\Gamma(k-1)}(x-\tau)^{k} d \tau .
$$

Como $f$ é contínua em $[a, b]$ e $\alpha>0,(x-\tau)^{\alpha} f(\tau)$ é limitado em $[0, b]$. Temos que a integral converge uniformemente, pois o somatório converge em $[0, b]$. Pelo Lema 2.1. é possível trocar a ordem do somatório com a integral. Assim ,temos,

$$
\begin{aligned}
{ }_{a} \mathrm{~J}_{x}^{\alpha} f(x)= & \sum_{k=0}^{\infty} \frac{(-1)^{k} f^{(k)}(x)}{\Gamma(k+1) \Gamma(\alpha)} \int_{a}^{x}(x-\tau)^{k+\alpha-1} d \tau \\
& =\sum_{k=0}^{\infty} \frac{(-1)^{k} f^{(k)}(x)}{\Gamma(\alpha) \Gamma(k+1)(k+\alpha)}(x-a)^{k+\alpha} \\
& =\sum_{k=0}^{\infty}\left(\begin{array}{c}
-\alpha \\
k
\end{array}\right) \frac{f^{(k)}(x)}{\Gamma(k+\alpha+1)}(x-a)^{k+\alpha}
\end{aligned}
$$

Lema 2.3 Seja $f(x)$ uma função analítica no intervalo $[a, b]$. Então a derivada de RiemannLiouville satisfaz a relação

$$
{ }_{a}^{\mathrm{RL}} \mathrm{D}_{x}^{\alpha} f(x)=\sum_{k=0}^{\infty} \frac{f^{(k)}(x)}{\Gamma(k-\alpha+1)}(x-a)^{k-a} .
$$

Demonstração. Para $0 \leq n-\alpha<1, n \in \mathbb{N}$, temos da definição (2.3) e do 2.2 que

$$
\begin{aligned}
{ }_{a}^{\mathrm{RL}} \mathrm{D}_{x}^{\alpha} f(x) & =D^{n}\left({ }_{a} \mathrm{~J}_{x}^{\alpha} f(x)\right) \\
& =D^{n}\left(\sum_{k=0}^{\infty}\left(\begin{array}{c}
\alpha-n \\
k
\end{array}\right) \frac{f^{(k)}(x)(x-a)^{k+n-\alpha}}{\Gamma(k+n-\alpha+1)}\right) .
\end{aligned}
$$


Usando a regra clássica de Leibniz (Equação 2.25), temos

$$
\begin{aligned}
\mathrm{RL}_{a} \mathrm{D}_{x}^{\alpha} f(x & =\sum_{k=0}^{\infty} \sum_{p=0}^{\infty}\left(\begin{array}{c}
\alpha-n \\
k
\end{array}\right)\left(\begin{array}{c}
n \\
p
\end{array}\right) \frac{f^{(p+k)}(x)(x-a)^{k+p-a}}{\Gamma(p+k-\alpha+1)} \\
& =\sum_{k+p=0}^{\infty}\left(\begin{array}{c}
\alpha \\
p+k
\end{array}\right) \frac{f^{(p+k)}(x)(x-a)^{k+p-a}}{\Gamma(p+k-\alpha+1)} \\
& =\sum_{k^{\prime}}^{\infty}\left(\begin{array}{c}
\alpha \\
k^{\prime}
\end{array}\right) \frac{f^{\left(k^{\prime}\right)}(x)(x-a)^{k^{\prime}-a}}{\Gamma\left(k^{\prime}-\alpha+1\right)} .
\end{aligned}
$$

A partir do Lema 2.3 podemos chegar à regra de Leibniz generalizada:

Teorema 2.5 [53] Sejam duas funções analíticas $f(x)$ e $g(x)$ em $[a, b]$, a Regra de Leibniz para a derivada de Riemann-Liouville é assegurada para $\alpha \in \mathbb{R}$, isto é,

$$
{ }_{a}^{\mathrm{RL}} \mathrm{D}_{x}^{\alpha}(f g)(x)=\sum_{k=0}^{\infty}\left(\begin{array}{l}
\alpha \\
k
\end{array}\right) f^{(k)}(x)\left({ }_{a}^{\mathrm{RL}} \mathrm{D}_{x}^{\alpha-k} g(x)\right) .
$$

Demonstração. Como $f$ e $g$ são funções analíticas, $(f g)$ também é analítica. Então, do Lema 2.3 . temos

$$
{ }_{a}^{\mathrm{RL}} \mathrm{D}_{x}^{\alpha}(f g)(x)=\sum_{k=0}^{\infty}\left(\begin{array}{l}
\alpha \\
k
\end{array}\right) \frac{(f g)^{(k)}}{\Gamma(k-\alpha+1)}(x-a)^{k-\alpha} .
$$

Usando a Equação 2.25, vamos obter

$$
\begin{aligned}
{ }_{a}^{\mathrm{RL}} \mathrm{D}_{x}^{\alpha}(f g)(x) & =\sum_{k=0}^{\infty}\left(\begin{array}{l}
\alpha \\
k
\end{array}\right) \frac{(x-a)^{k-\alpha}}{\Gamma(\alpha+k-1)} \sum_{p=0}^{k}\left(\begin{array}{l}
k \\
p
\end{array}\right) f^{(p)} g^{(k-p)} \\
& =\sum_{k=0}^{\infty} \sum_{p=0}^{k}\left(\begin{array}{l}
\alpha \\
k
\end{array}\right)\left(\begin{array}{l}
k \\
p
\end{array}\right) \frac{(x-a)^{k-\alpha}}{\Gamma(k-\alpha+1)} f^{(p)} g^{(k-p)}
\end{aligned}
$$

Lembrando que $\left(\begin{array}{l}\alpha \\ k\end{array}\right)\left(\begin{array}{l}k \\ p\end{array}\right)=\left(\begin{array}{l}\alpha \\ p\end{array}\right)\left(\begin{array}{l}\alpha-p \\ k-p\end{array}\right)$ e invertendo a ordem dos somatórios, temos

$$
\begin{aligned}
\mathrm{RL}_{a} \mathrm{D}_{x}^{\alpha}(f g)(x) & =\sum_{p=0}^{\infty} \sum_{k=p}^{\infty}\left(\begin{array}{l}
\alpha \\
p
\end{array}\right)\left(\begin{array}{l}
\alpha-p \\
k-p
\end{array}\right) \frac{(x-a)^{k-\alpha}}{\Gamma(k-\alpha+1)} f^{(p)} g^{(k-p)} \\
& =\sum_{p=0}^{\infty}\left(\begin{array}{l}
\alpha \\
k
\end{array}\right) f^{(p)} \sum_{k=p}^{\infty}\left(\begin{array}{l}
\alpha-p \\
k-p
\end{array}\right) \frac{g^{(k-p)}}{\Gamma((k-p)-(\alpha-p)+1)}(x-a)^{(k-p)-(\alpha-p)}
\end{aligned}
$$

Usando o Lema 2.3 , chegamos a

$$
\sum_{p=0}^{\infty}\left(\begin{array}{l}
\alpha \\
p
\end{array}\right) f^{(p)}\left({ }_{a}^{\mathrm{RL}} \mathrm{D}_{x}^{\alpha-p} g(x)\right)
$$

Infelizmente, não há uma representação simétrica pra a derivada de Caputo. Neste caso, a regra de Leibniz pode ser calculada utilizando a relação entre as derivadas de Caputo e Riemann-Liouville, 
dada no Teorema 2.4. Assim, utilizando o Teorema 2.5 na equação $(2.22)$, temos

$$
\begin{aligned}
{ }_{a}^{\mathrm{C}} \mathrm{D}_{x}^{\alpha}(f g)(x) & ={ }_{a}^{\mathrm{RL}} \mathrm{D}_{x}^{\alpha}(f(x) g(x))-\sum_{k=0}^{n-1} \frac{t^{k-\alpha}}{\Gamma(k+1-\alpha)}(f(x) g(x))^{(k)}(0) \\
& =\sum_{k=0}^{\infty}\left(\begin{array}{l}
\alpha \\
k
\end{array}\right)\left({ }_{a}^{\mathrm{RL}} \mathrm{D}_{x}^{\alpha-k} f(x)\right) g^{(k)}(x)-\sum_{k=0}^{n-1} \frac{t^{k-\alpha}}{\Gamma(k+1-\alpha)}(f(x) g(x))^{(k)}(0) .
\end{aligned}
$$

\subsection{Uma Interpretação física do Cálculo Fracionário}

Há muitas sugestões para interpretação do cálculo fracionário, que podem ser encontrados, por exemplo, em [48], [12] e [50]. Aqui iremos mostrar uma interpretação bastante útil do cálculo fracionário, encontrado em [39], principalmente como motivação do seu uso nas redes neurais de Hopfield. O núcleo dessas interpretações é o conceito de memória. Em geral, quando a saída de um sistema em cada momento depende apenas da entrada no tempo atual, tais sistemas são ditos sistemas sem memória. Por outro lado, quando o sistema tem que se lembrar de valores anteriores da entrada, a fim de determinar o valor atual da saída, tais sistemas são chamados de sistemas com memória. Por exemplo, considere um resistor de resistência $R$, obedecendo à lei de Ohm $U(t)=R I(t)$. Esse sistema é sem memória, pois o valor da tensão $U$ depende apenas do valor da corrente $I$ no instante $t$. Agora, suponha um capacitor com capacitância $C$. Neste caso, a tensão $U$ entre as placas no instante $t$ vale

$$
U(t)=\frac{1}{C} \int_{-\infty}^{t} I(\tau) d \tau
$$

onde $I(\tau)$ é a corrente que chega ao capacitor no instante $\tau$. Deste modo, para calcular $U(t)$, deve-se conhecer a corrente do capacitor em todo o seu passado, desde $-\infty$ até $t$.

Teorema 2.6 A seguinte relação entre a derivada de Caputo e a derivada de Riemann-Liouville é assegurada:

$$
{ }_{a}^{\mathrm{C}} \mathrm{D}_{t}^{\alpha} f(t)={ }_{a}^{\mathrm{RL}} \mathrm{D}_{t}^{\alpha}\left(f(t)-\sum_{k=0}^{n-1} \frac{t^{k}}{k !} f^{(k)}(0)\right)
$$

Demonstração. A Equação $(2.29)$ decorre diretamente da Equação $(2.22)$, usando a derivada da função potência Equação. (2.10) e a propriedade de linearidade da derivada:

$$
\begin{aligned}
{ }_{a}^{\mathrm{C}} \mathrm{D}_{t}^{\alpha} f(t) & ={ }^{\mathrm{RL}} \mathrm{D}_{t}^{\alpha} f(t)-\sum_{k=0}^{n-1} \frac{t^{k-\alpha}}{\Gamma(k+1-\alpha)} f^{(k)}(0) \\
& ={ }_{a}^{\mathrm{RL}} \mathrm{D}_{t}^{\alpha} f(t)-\sum_{k=0}^{n-1} \frac{D^{\alpha} t^{k}}{\Gamma(k+1)} f^{(k)}(0) \\
& ={ }_{a}^{\mathrm{RL}} \mathrm{D}_{t}^{\alpha}\left(f(t)-\sum_{k=0}^{n-1} \frac{t^{k}}{k !} f^{(k)}(0)\right) .
\end{aligned}
$$

A Equação 2.29 mostra que a derivada fracionária segundo Caputo incorpora os valores iniciais da função e de suas derivadas de ordens inteiras menores ou iguais a $n-1$.

Para mostrar que as derivadas fracionárias possuem memória, vamos supor uma função $Y(t)$, cujo valor em termos de $f(t)$ pode ser escrita da seguinte forma:

$$
Y(t)=\int_{0}^{t} \frac{(t-\tau)^{\alpha-1}}{\Gamma(\alpha)} f(\tau) d \tau
$$


isto é, $Y(t)$ pode ser vista como uma soma ponderada de energia que armazena a entrada anterior da função $f(t)$. Baseado na definição acima, tal sistema é um sistema com memória e em tais sistemas, a memória decai à taxa de $w(t)=\frac{t^{\alpha-1}}{\Gamma(\alpha)}$, pois $Y(t)=\int_{0}^{t}(w(t-\tau) f(\tau)) d \tau$.

Da definição 2.2 e da equação 2.19, a aplicação da derivada de Caputo de ordem $\alpha$ aos dois lados da Equação (2.30) leva a

$$
{ }_{0}^{\mathrm{C}} \mathrm{D}_{t}^{\alpha} Y(t)={ }_{0}^{\mathrm{C}} \mathrm{D}_{t}^{\alpha} J_{t}^{\alpha} f(t)=f(t) .
$$

Como resultado, a equação diferencial que modela a memória do sistema $Y(t)$ é descrita por uma derivada fracionária. Este fato é importante para o desenvolvimento do cálculo fracionário, pois isto implica que as derivadas fracionárias são melhores para descrever sistemas com memória. Do ponto de vista físico, o que a memória é, e como ela é definida em um sistema, depende de uma profunda compreensão dos fenômenos. Além disso, não há regras e métodos específicos para selecionar o tipo de derivada fracionária em uma modelagem [39].

\subsubsection{Interpretação dos extremos (limites à esquerda)}

Vimos que uma característica particular da integral e derivadas fracionárias ${ }_{a} \mathrm{~J}_{t}^{\alpha} f(t),{ }_{a}^{\mathrm{RL}} \mathrm{D}_{t}^{\alpha}$ e ${ }_{a}^{\mathrm{C}} \mathrm{D}_{t}^{\alpha}$ é o uso do extremo $a$. Suponha que esses operadores estão definidos no intervalo $[a, b], a \leq t \leq b$. As derivadas fracionárias que foram apresentadas até agora usam o limite à esquerda de $[a, b]$ como extremo, sendo então conhecidas como derivadas fracionárias à esquerda de $f(t)$. Seria possível definir estas derivadas com o limite superior $b$ do intervalo, o qual seriam as derivadas fracionárias à direita de $f(t)$, como por exemplo

$$
{ }_{t}^{\mathrm{RL}} \mathrm{D}_{b-}^{\alpha} f(t)=\frac{(-1)^{n}}{\Gamma(n-\alpha)} \frac{d^{n}}{d x^{n}} \int_{t}^{b}(\tau-t)^{n-\alpha-1} f(\tau) d \tau
$$

onde $n$ é o menor inteiro maior que $\alpha$. As noções de derivadas à esquerda e à direita podem ser consideradas do ponto de vista físico [48]. Sendo $t$ o tempo, $f(t)$ é um processo dinâmico que se desenvolve com o tempo. Se tivermos $a \leq \tau<t$, então o estado $f(\tau)$ pertence ao passado deste processo; analogamente, para $t<\tau_{1} \leq b, f\left(\tau_{1}\right)$ pertence ao futuro de $f$. Isso significa que a derivada fracionária à esquerda é aplicada em estados passados de $f$, enquanto que a derivada à direita usa estados futuros dessa função.

$$
{ }_{a}^{C} D_{t}^{\alpha} f(t) \quad \quad{ }_{t}^{C} D_{b-}^{\alpha} f(t)
$$

\section{Derivada à Esquerda Derivada à Direita}

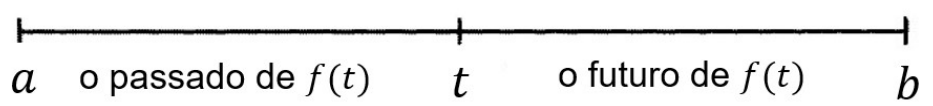

Figura 2.2: As derivadas à esquerda e à direita como operações no passado e futuro de $f(t)$

Assim, da interpretação de que derivadas fracionárias descrevem sistemas com memória, o estado atual $f(t)$ depende de todos os estados passados $f(\tau), a \leq \tau<t$ que será a memória do sistema. Fisicamente, sistemas que capturam memória do futuro violam o princípio da causalidade, motivo pelo qual as derivadas fracionárias à direita não serem utilizadas neste trabalho. 


\section{Capítulo 3}

\section{Estabilidade de Pontos de Equilíbrios de EDOFs}

Apresentaremos neste capítulo o critério de Mittag-Leffler para estabilidade de EDOFs, introduzido por Yan Li e outros [32] em 2009, que é uma generalização do método direto de Lyapunov. Para isso, será necessário estudar o método da transformada de Laplace para resolução de EDOFs e também o critério de existência e unicidade destas equações, que também serão apresentados neste capítulo.

\subsection{Função de Mittag-Leffler}

A função de Mittag-Leffler é uma função introduzida pelo matemático sueco Magnus Gustaf Mittag-Leffler, que a estudou em 1903. Esta função é uma generalização da função exponencial e desempenha papel importante no estudo das equações diferenciais de ordem fracionária. A função de Mittag-Leffler de um parâmetro é definida como

$$
E_{\alpha}(z)=\sum_{k=0}^{\infty} \frac{z^{k}}{\Gamma(\alpha k+1)}
$$

onde $\alpha>0$ e $z$ uma variável que pode ser complexa ou real. Iremos sempre supor que a variável $z$ é real neste texto. Também podemos definir a função de Mittag-Leffler de dois parâmetros, introduzida por Agarwal [25], da seguinte maneira

$$
E_{\alpha, \beta}(z)=\sum_{k=0}^{\infty} \frac{z^{k}}{\Gamma(\alpha k+\beta)}
$$

onde $\alpha, \beta>0$. Neste caso, temos $E_{\alpha, 1}(z)=E_{\alpha}(z)$. Note que, para o caso em que $\alpha=1$,

$$
E_{1}(z)=\sum_{k=0}^{\infty} \frac{z^{k}}{\Gamma(k+1)}=\sum_{k=0}^{\infty} \frac{z^{k}}{k !}=e^{z},
$$

ou seja, a função exponencial é um caso particular da função Mittag-Leffler. Também é de se esperar que as funções trigonométricas e hiperbólicas sejam casos particulares da função de Mittag-Leffler:

$$
\begin{aligned}
& E_{2,1}\left(z^{2}\right)=\sum_{k=0}^{\infty} \frac{z^{2 k}}{\Gamma(2 k+1)}=\sum_{k=0}^{\infty} \frac{z^{2 k}}{(2 k) !}=\cosh (z) \\
& E_{2,2}\left(z^{2}\right)=\sum_{k=0}^{\infty} \frac{z^{2 k}}{\Gamma(2 k+2)}=\sum_{k=0}^{\infty} \frac{z^{2 k}}{(2 k+1) !}=\frac{\operatorname{senh}(z)}{z} .
\end{aligned}
$$

Uma propriedade importante da função de Mittag-Leffler é que ela é completamente monotônica 
para $0<\alpha<1$, ou seja,

$$
(-1)^{m} \frac{d^{m}}{d x^{m}} E_{\alpha}(-x) \geq 0,
$$

com $x>0,0<\alpha<1$ e $m=0,1,2, \ldots$. Esta propriedade foi primeiramente estudada por Pollard [49], que demonstrou o seguinte teorema:

Teorema 3.1 Para $0 \leq \alpha \leq 1$, a função $E_{\alpha}(-x)$ é completamente monotônica.

Vamos discutir agora os domínios de definição das funções de Mittag-Leffler. Podemos dar um resultado completo para a versão de dois parâmetros, demonstrando que a função de Mittag-leffler é uniformemente convergente.

Lema 3.1 [12]) Considere a função de Mittag-Leffler de dois parâmetros $E_{\alpha, \beta}$ para $\alpha, \beta>0$. A série de potências definida pela Equação (3.2) é convergente para todo $z \in \mathbb{C}$.

Demonstração. Da equação 3.2 , temos

$$
E_{\alpha, \beta}(z)=\sum_{k=0}^{\infty} a_{k} z^{k} \operatorname{com} a_{k}=\frac{1}{\Gamma(\alpha k+\beta)} .
$$

Aplicaremos a fórmula de Stirling 1 , na qual afirma que, para $x \rightarrow \infty$,

$$
\Gamma(x+1)=\left(\frac{x}{e}\right)^{x} \sqrt{2 \pi x}(1+o(1)) .
$$

Desta forma, temos

$$
a_{k}^{1 / k}=\left(\frac{e}{\alpha k+\beta}\right)^{\alpha+\beta / k}(2 \pi(\alpha k+\beta))^{\frac{-1}{2 k}}(1+o(1)) \rightarrow 0
$$

quando $j \rightarrow \infty$, desde que $\alpha>0$. Então, pelo critério da raiz, o raio de convergência desta série de potências é infinito.

A função de Mittag-Leffler de dois parâmetros possui muita importância no cálculo fracionário, pois diversas relações para as equações fracionárias podem ser obtidas usando a técnica de transformação de Laplace.

\subsection{Transformada de Laplace}

A transformada de Laplace é fundamental para o estudo de equações diferenciais de ordem fracionária. Portanto, faremos nesta seção uma breve revisão da definição e algumas propriedades desta técnica.

Definição 3.1 (Transformada de Laplace) Seja $f(t)$ uma função real ou complexa, definida no intervalo $0 \leq t<\infty$ e $z=s+i v$ complexo e $F(z)$ uma função definida pela integral imprópria

$$
F(z)=\int_{0}^{\infty} f(t) e^{-z t} d t=\lim _{b \rightarrow+\infty}\left[\int_{0}^{b} f(t) e^{-z t} d t\right]
$$

então a função (3.4) recebe o nome de transformada de Laplace da função $f(t)$. Se z é real, usamos $z=s$ e a definiçấo fica simplesmente na forma

$$
\mathcal{L}\{f(t)\}=F(s)=\int_{0}^{\infty} f(t) e^{-s t} d t
$$

\footnotetext{
${ }^{1}$ Uma demonstração da Fórmula de Stirling pode ser encontrada em [45].
} 
Definição 3.2 (Ordem Exponencial) Uma função $f(t)$, definida para $t>0$, é de ordem exponencial $\alpha$ se existem constantes reais $M>0, t>0$ e $\alpha \in \Re$, tais que

$$
|f(t)| \leq M e^{a t}, t>t_{0}
$$

ou, da mesma forma, $e^{-a t}|f(t)| \leq M$ se, e somente se, $\lim _{t \rightarrow \infty}\left|f(t) e^{-a t}\right|=0$. Uma função $f(t)$ é limitada se existe $M>0$ tal que $|f(t)| \leq M$. Assim, se $f$ é limitada, temos

$$
e^{-a t}|f(t)| \leq M, t \geq 0, a>0
$$

Logo, toda função limitada é de ordem exponencial.

Teorema 3.2 (Existência da transformada de Laplace) [6] Se uma função $f(t)$, definida em $t \geq 0$, é contínua por partes em todo o intervalo fechado $0 \leq t \leq c$, com $c>0$ e de ordem exponencial, então existe $a \in \Re$ de modo que $\mathcal{L}(f(t))(s)$ existe para $\operatorname{Re}(s)>a$.

Demonstração. Como $f(t)$ é de ordem exponencial, existem constantes $M$ e $t_{0}$ tal que $|f(t)| \leq$ $M e^{a t}, t>t_{0}$. Escolhendo $T \geq t_{0}$, podemos escrever a transformada de Laplace como,

$$
\mathcal{L}\{(t)\}=\int_{0}^{\infty} f(t) e^{-s t} d t=\int_{0}^{T} f(t) e^{-s t} d t+\int_{T}^{\infty} f(t) e^{-s t} d t
$$

A primeira parcela do lado direito é uma integral definida no intervalo $[0, T]$, portanto está bem definido. Para a segunda parcela, como $T>t$, temos

$$
\begin{gathered}
\left|\int_{T}^{\infty} f(t) e^{-s t} d t\right| \leq \int_{T}^{\infty}\left|f(t) e^{-s t}\right| d t \leq \int_{T}^{\infty} M e^{a t} e^{s-t} d t \\
=M \int_{T}^{\infty} e^{-(s-a) t} d t=\frac{M}{s-a} e^{-(s-c) T}
\end{gathered}
$$

Como $\lim _{T \rightarrow \infty} \frac{M}{s-a} e^{-(s-a) T}=0$, segue que a integral $\int_{0}^{\infty} f(t) e^{-s t} d t$ converge para $s>a$.

Definição 3.3 (Convolução) Sejam $f(t)$ e $g(t)$ duas funções integráveis e de ordem exponencial $\alpha$ e $\beta$, com transformada de Laplace $F(s)$ e $G(s)$ em $[0, \infty)$, respectivamente. Definimos a convolução de $f(t)$ e $g(t)$, a qual denotaremos por $(f * g)(t)$ como sendo

$$
(f * g)(t)=\int_{0}^{t} f(t-\tau) g(\tau) d \tau=\int_{0}^{t} f(\tau) g(t-\tau) d \tau .
$$

Ao calcularmos a transformada de Laplace de um produto de convolução, temos

$$
\mathcal{L}\{(f * g)(t)\}=\int_{0}^{\infty} e^{-s t} \int_{0}^{t} f(\tau) g(t-\tau) d \tau d t .
$$

A integração acima está ocorrendo na seguinte região do plano $t \tau$ : que pode ser escrita como $0 \leq \tau \leq t, 0 \leq t<\infty$, ou $\tau \leq t<\infty, 0 \leq \tau<\infty$. Logo

$$
\int_{0}^{\infty} \int_{0}^{t} e^{-s t} f(\tau) g(t-\tau) d \tau d t=\int_{0}^{\infty} f(t) \int_{t}^{\infty} e^{-s t} g(t-\tau) d t d \tau
$$

Introduzindo a mudança de variável $u=t-\tau$ :

$$
\begin{gathered}
\int_{0}^{\infty} \int_{0}^{t} e^{-s t} f(\tau) g(t-\tau) d \tau d t=\int_{0}^{\infty} f(t) \int_{t}^{\infty} e^{-s(t+u} g(u) d u d \tau \\
=\int_{0}^{\infty} f(\tau) e^{-s \tau} d \tau \int_{0}^{\infty} g(u) e^{-s u} d \tau
\end{gathered}
$$




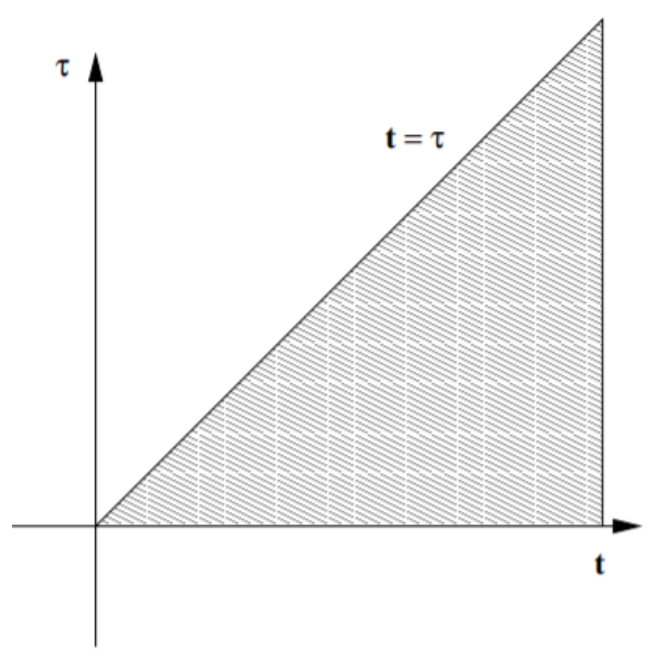

Figura 3.1: Plano t $\tau$.

logo, obtemos:

$$
\mathcal{L}\{(f * g)(t)\}=\mathcal{L}\{f(t)\} \mathcal{L}\{g(t)\} .
$$

Tendo aplicado a transformada de Laplace em um problema de valor inicial, temos que aplicar a transformada de Laplace inversa para recuperar a solução original do problema. O conceito desta técnica é dada na definição a seguir

Definição 3.4 (Transformada Laplace inversa) $S e F(s)=\mathcal{L}(f(t))$ é a transformada de Laplace de $f(t)$, então dizemos que $f(t)=\mathcal{L}^{-1}\{F(s)\}$ é a transformada de Laplace inversa da função $F(s)$, que pode ser definida pela integral complexa de Bromwich

$$
\frac{1}{2 \pi} \int_{\gamma-i \infty}^{\gamma+i \infty} F(s) e^{s t} d s
$$

onde a integração é efetuada ao longo da reta $s=\gamma$ no plano complexo em que $\gamma$ é menor que a parte real de todas as singularidades.

A Definição 3.4 só faz sentido quando a transformação definida no conjunto de funções que possuem transformada de Laplace for bijetora, ou seja, cada função $f(t)$ está relacionada a uma única transformada $F(s)$.

\subsection{Transformada de Laplace para o cálculo fracionário}

Aqui calcularemos as transformadas de Laplace de algumas funções, baseado em [2] e [48] que serão úteis no desenvolvimento da teoria para solução e estabilidade de EDOF's. Vamos começar com o seguinte lema:

Lema 3.2 A transformada de Laplace da integral de Riemann-Liouville é dada por $\mathcal{L}\left\{J_{t}^{\alpha} f(t)\right\}=$ $s^{-\alpha} \mathcal{L}\{f(t)\}$.

Demonstração. Vamos definir a função $\phi_{\alpha}(t)=\frac{t^{\alpha-1}}{\Gamma(\alpha)}$. Note que

$$
\mathcal{L}\left\{\phi_{\alpha}(t)\right\}=\int_{0}^{\infty} e^{-s t} \frac{t^{\alpha-1}}{\Gamma(\alpha)} d t=\frac{1}{\Gamma(\alpha)} \int_{0}^{\infty} e^{-s t} t^{\alpha-1} d t
$$


Considerando a substituição $u=s t$, temos

$$
\begin{aligned}
\mathcal{L}\left\{\phi_{\alpha}(t)\right\} & =\lim _{b \rightarrow \infty} \frac{1}{\Gamma(\alpha)} \int_{0}^{b} e^{-u} \frac{u^{\alpha-1}}{s^{\alpha-1}} \frac{d u}{s} \\
& =\lim _{b \rightarrow \infty} \frac{1}{s^{\alpha} \Gamma(\alpha)} \int_{0}^{b} e^{-u} u^{\alpha-1} d u \\
& =\frac{1}{\Gamma(\alpha) s^{\alpha}} \Gamma(\alpha)=s^{-\alpha} .
\end{aligned}
$$

Usando o produto de convolução, vamos obter

$$
\begin{aligned}
\mathcal{L}\left\{J_{t}^{\alpha} f(t)\right\} & =\mathcal{L}\{(\phi \star f)(t)\} \\
& =\mathcal{L}\left\{\phi_{\alpha}(t)\right\} \mathcal{L}\{f(t)\} \\
& =s^{\alpha} \mathcal{L}\{f\}
\end{aligned}
$$

A transformada de Laplace da função do Lema (3.3) é bastante utilizada na resolução de equações diferenciais fracionárias e será aplicada para demonstração do Teorema 3.11 que iremos enunciar mais a frente.

Lema 3.3 Seja $\alpha, \beta>0$. A transformada de Laplace da função $z^{\alpha+\beta-1} \frac{d^{k} E_{\alpha, \beta}\left( \pm a z^{\alpha}\right)}{d\left( \pm a z^{\alpha}\right)^{k}}$,

$$
\mathcal{L}\left\{z^{\alpha+\beta-1} \frac{d^{k} E_{\alpha, \beta}\left( \pm a z^{\alpha}\right.}{d\left( \pm a z^{\alpha}\right)^{k}}\right\}=\frac{k ! s^{\alpha-\beta}}{\left(s^{\alpha} \mp a\right)^{k+1}}
$$

Demonstração. Vamos primeiramente considerar a seguinte integral

$$
\int_{0}^{\infty} e^{-t} t^{\beta-1} E_{\alpha, \beta}\left( \pm \mu t^{\alpha}\right) d t=\int_{0}^{\infty} e^{-t} t^{\beta-1} \sum_{k=0}^{\infty} \frac{( \pm \mu)^{k} t^{\alpha k}}{\Gamma(\alpha k+\beta)} d t .
$$

Devido à convergência uniforme da função de Mittag-Leffler, podemos trocar o somatório com a integral

$$
\int_{0}^{\infty} e^{-t} t^{\beta-1} E_{\alpha, \beta}\left( \pm \mu t^{\alpha}\right) d t=\sum_{0}^{\infty} \frac{( \pm \mu)^{k}}{\Gamma(\alpha k+\beta)} \int_{0}^{\infty} e^{-t} t^{\alpha k+\beta-1} d t .
$$

Da definição da função gama, temos

$$
\int_{0}^{\infty} e^{-t} t^{\alpha k+\beta-1} d t=\Gamma(\alpha k+\beta)
$$

Logo, podemos escrever 3.12 como,

$$
\int_{0}^{\infty} e^{-t} t^{\beta-1} E_{\alpha, \beta}\left( \pm \mu t^{\alpha}\right) d t=\sum_{k=0}^{\infty} \frac{( \pm \mu)^{k}}{\Gamma(\alpha k+\beta)} \Gamma(\alpha k+\beta)=\frac{1}{1 \mp \mu} .
$$

supondo que $|\mu|<1$, pois caso contrário a série acima divergiria. Diferenciando $k$-vezes a expressão 3.13 temos

$$
\begin{gathered}
\frac{d^{k}}{d \mu^{k}} \int_{0}^{\infty} e^{-t} t^{\beta-1} E_{\alpha, \beta}\left( \pm \mu t^{\alpha}\right) d t=\frac{k !( \pm 1)^{k}}{(1 \mp \mu)^{k+1}} \\
\int_{0}^{\infty} e^{-t} t^{\beta-1}\left( \pm t^{\alpha}\right)^{k} \frac{d^{k}}{d\left(\mp \mu t^{\alpha}\right)^{k}} E_{\alpha, \beta}\left( \pm t^{\alpha}\right) d t=\frac{k !( \pm 1)^{k}}{(1 \mp \mu)^{k+1}}
\end{gathered}
$$

Introduzindo a mudança de variável $z$ com $s t$

$$
\frac{k !( \pm 1)^{k}}{(1 \mp \mu)^{k+1}}=\int_{0}^{\infty} e^{-s z} s^{\beta-1} z^{\beta-1}( \pm 1)^{k} s^{\alpha k} z^{\alpha k} \frac{d^{k} E_{\alpha, \beta}\left( \pm \mu s^{\alpha} z^{\alpha}\right)}{d\left( \pm \mu s^{\alpha} z^{\alpha}\right)^{k}} s d z
$$


Substituindo $\mu s^{\alpha}$ por $a$, segue

$$
\frac{k !}{s^{\beta} s^{a k}\left(1 \mp \frac{a}{s^{\alpha}}\right)^{k+1}}=\int_{0}^{\infty} e^{-s z} z^{\alpha k+\beta-1} \frac{d^{k} E_{\alpha, \beta}\left( \pm a z^{\alpha}\right)}{d\left( \pm a z^{\alpha}\right)^{k}} d z,
$$

que demonstra o teorema, já que o lado direito é a trasnformada de Laplace da função que queríamos calcular.

Se substituirmos $k=0$ em (3.14), temos que

$$
\mathcal{L}\left\{t^{\beta-1} E_{\alpha, \beta}\left( \pm a t^{\alpha}\right)\right\}=\frac{s^{\alpha-\beta}}{s^{\alpha} \mp a} .
$$

Se $\alpha=\beta$ :

$$
\mathcal{L}\left\{t^{\beta-1} E_{\alpha, \alpha}\left( \pm a t^{\alpha}\right)\right\}=\frac{1}{s^{\alpha} \mp a}
$$

Uma propriedade útil que irá ser usada para demonstrar as transformadas de Laplace das derivadas de Riemann-Liouville e Caputo é a transofrmada de Laplace da derivada de ordem $n$ inteira da função:

$$
\mathcal{L}\left\{f^{n}(t)\right\}=s^{n} f(s)-\sum_{k=0}^{n-1} s^{n-k-1} f^{k}(0)=s^{n} f(s)-\sum_{k=0}^{n-1} s^{k} f^{n-k-1}(0),
$$

que é obtida pela integração por partes na integral da transformada de Laplace, admitindo que esta integral existe.

Lema 3.4 A transformada de Laplace da derivada de Caputo é dada por

$$
\mathcal{L}\left\{{ }_{a}^{\mathrm{C}} \mathrm{D}_{t}^{\alpha} f(t)\right\}=s^{\alpha} f(s)-\sum_{k=0}^{n-1} f^{k} s^{\beta-k-1}
$$

sendo $n$ o menor inteiro maior que $\alpha>0$.

Demonstração: Da definição da derivada de Caputo, temos

$$
\mathcal{L}\left\{{ }_{a}^{\mathrm{C}} \mathrm{D}_{t}^{\alpha} f(t)\right\}=\mathcal{L}\left\{\mathrm{J}_{t}^{n-\alpha}\left(D^{n} f(t)\right)\right\} .
$$

Aplicando a transformada de Laplace da integral de Riemann-Lioville e da derivada de ordem inteira, vamos obter

$$
\begin{aligned}
\mathcal{L}\left\{{ }_{a}^{\mathrm{C}} \mathrm{D}_{t}^{\alpha} f(t)\right\} & =s^{-n+\alpha} \mathcal{L}\left\{D^{n} f(t)\right\} \\
& =s^{-n+\alpha}\left(s^{n} \mathcal{L}\{f(t)\}-\sum_{k=0}^{n-1} f^{k}(0) s^{n-1-k}\right) \\
& =s^{\alpha} f(s)-s^{-n+\alpha} \sum_{k=0}^{n-1} f^{k}(0) s^{n-1-k} \\
& =s^{\alpha} f(s)-\sum_{k=0}^{n-1} f^{k}(0) s^{\alpha-k-1} .
\end{aligned}
$$

De forma parecida podemos mostrar o seguinte Lema:

Lema 3.5 A transformada de Laplace da derivada fracionária de Riemann-Liouville é dada por

$$
\mathcal{L}\left\{{ }^{R L} D_{t}^{\alpha} f(t)\right\}=s^{\alpha} F(s)-\sum_{k=0}^{n-1} s^{n-k-1} D^{k-n+\alpha} f(0)
$$


onde $n$ é o menor inteiro maior que $\alpha>0$.

Demonstração: Da definição da derivada de Riemann-Liouville e usando a transformada de Laplace para derivadas, temos

$$
\begin{aligned}
\mathcal{L}\left\{{ }_{a}^{\mathrm{RL}} \mathrm{D}_{t}^{\alpha} f(t)\right\} & =\mathcal{L}\left\{D^{n} J_{t}^{n-\alpha} f(t)\right\} \\
& =s^{n} \mathcal{L}\left\{J_{t}^{n-\alpha} f(t)\right\}-\sum_{k=0}^{n-1} s^{n-k-1} D^{k}\left[J^{n-\alpha} f(0)\right] \\
& =s^{n}\left[s^{-(n-\alpha)} F(s)\right]-\sum_{k=0}^{n-1} s^{n-k-1} D^{k-(n-\alpha)} f(0) \\
& =s^{\alpha} F(s)-\sum_{k=0}^{n-1} s^{n-k-1} D^{k-n+\alpha} f(0) .
\end{aligned}
$$

\subsection{Solução de uma EDOF}

As EDOFs aparecem cada vez mais em várias áreas de pesquisa e em aplicações na engenharia. Há diversos métodos para resolver estas equações mas, conforme explica Podlubny [48], métodos conhecidos possuem certas desvantagens. Métodos descritos em [43] e [29] por exemplo, funcionam em equações de ordem racional, mas não são aplicadas no caso em que a ordem de derivação é um número real. Ainda há o método iterativo dado em [53], que permite a solução de equações diferenciais fracionárias de ordem real arbitrária, mas funciona eficazmente apenas para equações relativamente simples, tal como ocorre com o método de séries [43].

Para contornar todos estes problemas, iremos utilizar neste texto um método que pode ser aplicado a uma grande variedade de problemas de valor inicial para equações diferenciais de ordem fracionária, e que também é muito conhecido para resolver equações diferenciais de ordem inteira. O método usa transformadas de Laplace, baseando-se na transformada inversa para se chegar numa solução em termos da função de Mittag-Leffler de dois parâmetros $E_{\alpha, \beta}(z)$.

Vamos dar um exemplo de como resolver uma equação diferencial ordinária de ordem fracionária com derivada de Riemann-Liouville, com o uso da transformada de Laplace.

Exemplo 3.1 Resolver a equação ${ }_{a}^{R L} D_{t}^{\alpha} x(t)=a x(t)$, com condição inicial $x_{0}=c$, onde a é constante e $0<\alpha \leq 1$ um número real.

Solução: Usando a transformada de Laplace em ambos os lados da equação, temos

$$
\begin{gathered}
\mathcal{L}\left\{{ }^{R L} D_{t}^{\alpha} x(t)\right\}=\mathcal{L}\{a x(t)\} \\
s^{\alpha} X(s)-J_{t}^{1-\alpha} x(0)=a X(s) .
\end{gathered}
$$

A constante $J_{t}^{1-\alpha} x(0)$ é o valor de $J_{t}^{1-\alpha} x(t)$ onde $t=0$. Vamos admitir que esse valor existe e chamá-lo de $c_{1}$. Prosseguindo, temos

$$
X(s)=\frac{c_{1}}{s^{\alpha}-a} .
$$

Usando a transformada de Laplace inversa desta função, chegamos em

$$
x(t)=\mathcal{L}^{-1}\left\{\frac{c_{1}}{s^{v}-a}\right\}=c_{1} t^{\alpha-1} E_{\alpha, \alpha}\left(a t^{\alpha}\right) .
$$

Nós gostaríamos de usar a condição inicial dada para encontrar o valor de $c_{1}$. Como

$$
\lim _{t \rightarrow 0^{+}} t^{\alpha-1} E_{\alpha, \alpha}\left(a t^{\alpha}\right)=1,
$$


então, da Equação (3.17), temos que $\lim _{t \rightarrow 0^{+}} x(t)=c_{1}$ o que leva a conclusão de que

$$
x_{0}=J^{1-\alpha} x(0) .
$$

É natural perguntar-se qual o significado físico de uma condição inicial com um integral de ordem fracionária. Infelizmente, até o momento, não há uma interpretação física para este problema, e esta é a principal dificuldade das EDO's fracionárias de Riemann-Liouville quando aplicamos a transformada de Laplace para o extremo à esquerda $t=0$.

Felizmente, isso não ocorre para o caso em que a derivada da EDO fracionária é de Caputo, pois observando a Equação (3.15), a transformada de Laplace da derivada de Caputo envolve apenas derivadas de ordem inteira. Assim, a transformada de Laplace da derivada de Caputo envolve a função $f(t)$ e suas derivadas no extremo $t=0$, para as quais existe uma certa interpretação física (por exemplo, $f(0)$ é a posição inicial, $f^{\prime}(0)$ é a velocidade inicial. e $f^{\prime \prime}(0)$ é a aceleração inicial). Podemos concluir que a derivada de Caputo pode ser usada para resolver problemas aplicados que conduzem a EDOFs com coeficientes constantes e condições iniciais da forma tradicional. Esse é o motivo das redes neurais de Hopfield de ordem fracionária e de muitas outras aplicações em física, química e engenharia serem estudadas utilizando a derivada de Caputo.

\subsection{Existência e Unicidade de EDOFs}

Já é bem conhecido as condições para que um problema de valor inicial (Problema de Cauchy) satisfaça as condições de existência e unicidade. Estamos interessados agora nas condições de existência e unicidade de EDO's que envolvem ordem fracionária. Para este tipo de discussão, nos baseamos em [12], que faz uma ótima exposição do assunto. O próximo teorema é o teorema de existência e da unicidade para as equações baseadas na derivada de Riemann-Liouville. Sem perda de generalidade, admitimos neste resultado que as derivadas fracionárias são desenvolvidas supondo que o extremo inferior dos operadores de Riemann-Liouville e Caputo são iguais a zero e para simplificar a notação omitiremos o extremo zero da notação.

Teorema 3.3 [12] Sejam $\alpha>0, \alpha \notin \mathbb{N}$ e $n=\lceil\alpha\rceil$, onde $\lceil n\rceil$ é o menor inteiro maior que $n$. Além disso, seja $K>0, h^{*}>0$, e $b_{1}, b_{2}, \ldots, b_{n} \in \mathbb{R}$. Defina

$$
G:=\left\{(t, x) \in \Re^{2}: 0 \leq t \leq h^{*}, x \in \Re \text { para } t=0 \text { e }\left|t^{n-\alpha} x-\frac{\sum_{k=1}^{n} b_{k} t^{n-\alpha}}{\Gamma(\alpha-k+1)}\right|<K \text { caso contrário }\right\},
$$

e admita que a função $f: G \rightarrow \Re$ é contínua e limitada em $G$ e satisfaz a condição de Lipschitz com respeito à segunda variável, isto é

$$
\left|f\left(t, x_{1}\right)-f\left(t, x_{2}\right)\right|<L\left|x_{1}-x_{2}\right| .
$$

Então a equação diferencial

$$
\mathrm{RL}^{\alpha} x(t)=f(t, x(t))
$$

munida das condições iniciais

$$
{ }^{\mathrm{RL}} \mathrm{D}_{t}^{\alpha-k} x(0)=b_{k}(k=1,2, \ldots, n-1), \quad \lim _{z \rightarrow 0^{+}} J^{n-\alpha} x(z)=b_{n},
$$

tem uma solução contínua unicamente definida $x \in(0, h]$ onde

$$
h:=\min \left\{h^{*}, \tilde{h},\left(\frac{\Gamma(\alpha+1) K}{M}\right)^{1 / n}\right\}
$$


com $M:=\sup _{(t, z) \in G}|f(t, z)|$ e $\tilde{h}$ sendo um número positivo arbitrário satisfazendo a restrição

$$
\tilde{h}<\left(\frac{\Gamma(2 \alpha-n+1)}{\Gamma(\alpha-n+1) L}\right)^{1 / \alpha}
$$

Podemos ver que este resultado é muito similar aos resultados clássicos de existência e unicidade para equações de primeira ordem. A ideia para demonstração é transformar este problema de valor inicial em uma equação integral de Volterra (Lema 3.6). e então provar a existência e unicidade da solução desta integral usando alguns dos teoremas clássicos de ponto fixo da análise funcional.

Lema 3.6 Admita que as hipóteses do Teorema 3.3 e seja $h>0$. A função $x \in C(0, h]$ é solução da equação diferencial

$$
\begin{gathered}
{ }^{\mathrm{RL}} \mathrm{D}_{t}^{\alpha} x(t)=f(t, x(t)) \\
{ }^{\mathrm{RL}} \mathrm{D}_{t}^{\alpha-k} x(0)=b_{k}(k=1,2, \ldots, n-1), \quad \lim _{z \rightarrow 0^{+}} J^{n-\alpha} x(z)=b_{n},
\end{gathered}
$$

se e somente se é solução da equação integral de Volterra

$$
x(t)=\sum_{k=1}^{n} \frac{b_{k} t^{\alpha-k}}{\Gamma(\alpha-k+1)}+\frac{1}{\Gamma(\alpha)} \int_{0}^{t}(t-\tau)^{\alpha-1} f(\tau, x(\tau)) d \tau .
$$

Demonstração. Podemos escrever a Equação 3.19 como

$$
x(t)=\sum_{k=1}^{n} \frac{b_{k} t^{\alpha-k}}{\Gamma(\alpha-k+1)}+J_{t}^{\alpha}(t, x(t)) .
$$

Aplicando o operador de Riemann-Liouville em ambos os lados da equação e, devido à lei dos expoentes para a integral fracionária (2.2), temos que, para $1 \leq k \leq n-1$,

$$
{ }^{\mathrm{RL}} \mathrm{D}_{t}^{\alpha-k} x(t)=\sum_{j=1}^{n} \frac{b_{j}{ }^{\mathrm{RL}} \mathrm{D}_{t}^{\alpha-k} t^{\alpha-j}}{\Gamma(\alpha-j+1)}+{ }^{\mathrm{RL}} \mathrm{D}_{t}^{\alpha-k} J_{t}^{\alpha-k} J_{t}^{k} f(t, x(t)) .
$$

Pela equação (2.9),

$$
\sum_{j=1}^{n}{ }^{\mathrm{RL}} \mathrm{D}_{t}^{\alpha-k} t^{\alpha-j}=\sum_{j=1}^{n} \frac{\Gamma(\alpha-j+1)}{\Gamma(-j+n+1)} D_{t}^{n-k} t^{n-j},
$$

e se $j>k$, o somatório acima se anula identicamente. Se $t=0$, o somatório se anula para $j<k$ também. Então, da Equação (3.21), obtemos

$$
\mathrm{RL}_{t}^{\alpha-k} x(0)=\frac{b_{k}{ }^{\mathrm{RL}} \mathrm{D}_{0}^{\alpha-k}(t)^{\alpha-k}}{\Gamma(\alpha-k+1)}+J_{0}^{k}(t, x(t))
$$

Desde que $k \geq 1$, a integral fracionária se anula e aplicando o resultado do Exemplo 2.2 temos que

$$
{ }^{\mathrm{RL}} \mathrm{D}_{t}^{\alpha-k} t^{\alpha-k}=\Gamma(\alpha-k+1) .
$$

Então ${ }^{\mathrm{RL}} \mathrm{D}_{t}^{\alpha-k} x(0)=b_{k}$ deverá ser a condição inicial do problema. Finalmente, para o caso em que $k=n$, se usarmos o operador $J^{n-\alpha}$ em ambos os lados da Equação (3.19) e aplicarmos o limite para $z \rightarrow 0$ temos que todas as somas do somatório são nulas exceto para a $n$-ésima soma. Então

$$
\lim _{z \rightarrow 0} J_{z}^{n-\alpha} x(z)=\lim _{z \rightarrow 0}\left(J_{z}^{n-\alpha} \frac{b_{n} z^{\alpha-n}}{\Gamma(\alpha-n+1)}+J_{z}^{n-\alpha} J_{z}^{\alpha}(z, x(z))\right) .
$$

A integral ${ }_{0} \mathrm{~J}_{z}^{n-\alpha}{ }_{0} \mathrm{~J}_{z}^{\alpha}(z, x(z))={ }_{0} \mathrm{~J}_{z}^{n}(z, x(z)) \rightarrow 0$ quando $z \rightarrow 0$. Lembrando do Exemplo 2.1, ao 
aplicarmos para calcular a integral em (3.23), temos que

$$
\lim _{z \rightarrow 0+} J_{z}^{n-\alpha} x(z)=\lim _{z \rightarrow 0+} J_{z}^{n-\alpha} \frac{b_{n} J_{z}^{n-\alpha} z^{\alpha-n}}{\Gamma(\alpha-m+1)}=b_{n} .
$$

Consequentemente $x$ resolve o problema de valor inicial (3.18). Agora, se $x$ é uma solução contínua do problema de valor inicial (3.18), então nós definimos $z(t):=f(t, x(t))$. Por suposição, $z$ é uma função contínua e $z(t)=f(t, x(t))={ }^{\mathrm{RL}} \mathrm{D}_{t}^{\alpha} x(t)=D^{n} J_{t}^{n-\alpha} x(t)$ também é uma função contínua, isto é, $J_{t}^{n-\alpha} x(t) \in C^{n}(0, h]$. Do Teorema 2.3, o seguinte resultado é válido

$$
x(t)=J_{t}^{\alpha \mathrm{RL}} \mathrm{D}_{t}^{\alpha} x(t)+\lim _{t \rightarrow 0+} \sum_{k=1}^{n} \frac{t^{\alpha-k} D^{n-k+1} J^{n-\alpha} x(t)}{\Gamma(\alpha-k+1)} .
$$

Se aplicarmos as condições inicias acimas à Equação (3.25), obtemos

$$
x(t)=J_{t}^{\alpha \mathrm{RL}} \mathrm{D}_{t}^{\alpha} x(t)+\sum_{k=1}^{n} c_{k} x^{\alpha-k}=J_{t}^{\alpha} f(t, x(t))+\sum_{k=1}^{n} c_{k} x^{\alpha-k},
$$

com constantes $c_{1}, c_{2}, \ldots, c_{n}$, e $c_{k}=\frac{b_{k}}{\Gamma(\alpha-k+1)}$.

Uma observação é que $y$ é contínua no intervalo em $(0, h]$, e não no intervalo fechado $[0, h]$. Se fosse, a integral no lado direito da Equação (3.19) teria que ser contínua neste intervalo, devido à continuidade de $f$, e o somatório também. Da definição de $n$, podemos ver que o somatório é contínuo no intervalo $[0, h]$ para $k=1,2, \ldots, n-1$, mas não para $k=n$, pois $x^{\alpha-n} \rightarrow \infty$ quando $x \rightarrow 0$, pois $n>\alpha$ se, a menos que $b_{n}=0$.

Demonstração do Teorema 3.3. A ideia para a demonstração deste teorema é definir um conjunto $B$ e um operador $A \in B$ tal que se $x \in B \Rightarrow A x \in B$, a equação integral de Volterra (3.19) será escrita como $x=A x$. Com isso, satisfazendo as condições do teorema do ponto fixo de Weissinger (veja Apêndice A), mostramos que o operador $A$ tem um único ponto fixo $x^{*}$.

Vamos definir o seguinte conjunto

$$
B:=\left\{x \in C(0, h]: \sup _{0<t \leq h}\left|t^{n-\alpha} x(t)-\sum_{k=1}^{n} \frac{b_{k} t^{n-k}}{\Gamma(\alpha-k+1)}\right| \leq K\right\}
$$

e o operador $A x$

$$
A x(t):=\sum_{k=1}^{n} \frac{b_{k} t^{\alpha-k}}{\Gamma(\alpha-k+1)}+\frac{1}{\Gamma(\alpha)} \int_{0}^{t}(t-\tau)^{\alpha-1} f(\tau, x(\tau)) d \tau
$$

Se $x \in B$, obviamente $A x \in C(0, h]$. Além disso, lembrando que $M:=\sup _{(t, x) \in G}|f(t, x)|$

$$
\begin{aligned}
\left|t^{n-\alpha} A x(t)-\sum_{k=1}^{n} \frac{b_{k} t^{n-k}}{\Gamma(\alpha-k+1)}\right| & =\left|\frac{t^{n-\alpha}}{\Gamma(\alpha)} \int_{0}^{t}(t-\tau)^{\alpha-1} f(\tau, x(\tau)) d \tau\right| \\
& \leq \frac{t^{n-\alpha}}{\Gamma(\alpha)} M \int_{0}^{t}(t-\tau)^{\alpha-1} d \tau \leq \frac{t^{n-\alpha}}{\Gamma(\alpha)} M \frac{t^{\alpha}}{\alpha} \\
& =\frac{t^{\alpha} M}{\Gamma(\alpha+1)} \leq K,
\end{aligned}
$$

na qual a última desigualdade segue da definição de $h$, que para $t \in C(0, h]$

$$
t \leq h \leq\left(\frac{\Gamma(\alpha+1) K}{M}\right)^{1 / n} \leq\left(\frac{\Gamma(\alpha+1) K}{M}\right)^{1 / \alpha}
$$

Assim, chegamos ao resultado de que se $x \in B$ então $A x \in B$. Vamos agora introduzir um novo 
conjunto

$$
\hat{B}:=\left\{x \in C(0, h]: \sup _{0<t \leq h}\left|t^{n-\alpha} x(t)\right|<\infty\right\}
$$

e a seguinte norma

$$
\|x\|_{\hat{B}}:=\sup _{0<t \leq h}\left|t^{n-\alpha} x(t)\right| .
$$

Para verificar que $\hat{B}$ equipado com esta norma é um espaço normado, basta verificar que :

- 1) Se $\|x\|_{\hat{B}}=0$, como $t \neq 0$, temos que ter $x=0$;

- 2) $\left\|x_{1}+x_{2}\right\|_{\hat{B}}=\sup _{0<t \leq h}\left|t^{n-\alpha}\left(x_{1}(t)+x_{2}(t)\right)\right| \leq \sup _{0<t \leq h}\left|t^{n-\alpha} x_{1}(t)\right|+\sup _{0<t \leq h}\left|t^{n-\alpha} x_{2}(t)\right|=$ $\left\|x_{1}\right\|_{\hat{B}}+\left\|x_{2}\right\|_{\hat{B}}<\infty$

- 3) $\|\lambda x\|_{\hat{B}}=\sup _{0<t \leq h}\left|t^{n-\alpha} \lambda x(t)\right|=\lambda \sup _{0<t \leq h}\left|t^{n-\alpha} x(t)\right|=\lambda\|x\|_{\hat{B}}<\infty$,

para $\lambda>0$ constante. Temos também que $B$ é um subconjunto completd ${ }^{2}$ desse espaço. Usando agora a definição do operador $A$, podemos escrever a equação integral de Volterra de forma mais compacta como

$$
x=A x .
$$

Chegamos agora à última etapa da demonstração, onde é suficiente mostrar que o operador $A$ tem um único ponto fixo. Para este propósito, empregaremos o teorema do ponto fixo de Weissinger, ou seja, provaremos que, para $x, \tilde{x} \in B$,

$$
\left\|A^{j} x-A^{j} \tilde{x}\right\|_{\hat{B}} \leq\left(\frac{L h^{\alpha} \Gamma(\alpha-n+1)}{\Gamma(2 \alpha-n+1)}\right)^{j}\|x-\tilde{x}\|_{\hat{B}} .
$$

Este resultado pode ser demonstrado por indução. Para $j=0$ é trivial. A etapa de $j-1 \rightarrow j$, segue de

$$
\begin{aligned}
\left\|A^{j} x-A^{j} \tilde{x}\right\|_{\hat{B}} & \left.=\sup _{0<t \leq h} \mid t^{n-\alpha}\left(A^{j} x(t)-A^{j} \tilde{(} x\right)(t)\right) \mid \\
& =\sup _{0<x \leq h}\left|t^{n-\alpha}\left(A A^{j-1} x(t)-A A^{j-1} \tilde{x}(t)\right)\right| \\
& =\sup _{0<t \leq h} \frac{t^{n-\alpha}}{\Gamma(\alpha)}\left|\int_{0}^{t}(t-\tau)^{\alpha-1}\left[f\left(\tau, A^{j-1} x(\tau)\right)-f\left(\tau, A^{j-1} \tilde{x}(\tau)\right)\right] d \tau\right| \\
& \leq \sup _{0<t \leq h} \frac{t^{n-\alpha}}{\Gamma(\alpha)} \int_{0}^{t}(t-\tau)^{\alpha-1}\left|f\left(\tau, A^{j-1} x(\tau)\right)-f\left(\tau, A^{j-1} \tilde{x}(\tau)\right)\right| d \tau \\
& \leq \frac{L}{\Gamma(\alpha)} \sup _{0<t \leq h} t^{n-\alpha} \int_{0}^{t}(t-\tau)^{\alpha-1}\left|A^{j-1} x(\tau)-A^{j-1} \tilde{\tau}\right| d \tau,
\end{aligned}
$$

que decorre do fato de $f$ ser Lipschtiziana. Continuando,

$$
\begin{aligned}
\left\|A^{j} x-A^{j} \tilde{x}\right\|_{\hat{B}} & \leq \frac{L}{\Gamma(\alpha)} \sup _{0<t \leq h} t^{n-\alpha} \int_{0}^{t}(t-\tau)^{\alpha-1}\left|A^{j-1} x(\tau)-A^{j-1} \tilde{\tau}\right| d \tau \\
& \leq \frac{L}{\Gamma(\alpha)} \sup _{0<t \leq h} t^{n-\alpha} \int_{0}^{t}(t-\tau)^{\alpha-1} \tau^{\alpha-n} \tau^{n-\alpha}\left|A^{j-1} x(\tau)-A^{j-1} \tilde{\tau}\right| d \tau \\
& \leq \frac{L}{\Gamma(\alpha)}\left\|A^{j-1} x-A^{j-1} \tilde{x}\right\|_{\hat{B}} \sup _{0<t \leq h} t^{\alpha-n} \int_{0}^{t}(t-\tau)^{\alpha-1} \tau^{\alpha-n} d \tau \\
& =\frac{L}{\Gamma(\alpha)}\left\|A^{j-1} x-A^{j-1} \tilde{x}\right\|_{\hat{B}} \sup _{0<t \leq h} t^{\alpha-n} \frac{\Gamma(\alpha) \Gamma(\alpha-n+1)}{\Gamma(2 \alpha-n+1)} t^{\alpha},
\end{aligned}
$$

\footnotetext{
${ }^{2} \mathrm{Um}$ conjunto completo é um espaço métrico no qual cada sequência de Cauchy desse conjunto converge.
} 
onde a integral foi calculada baseando-se no Exemplo 2.1. Assim, temos

$$
\left\|A^{j} x-A^{j} \tilde{x}\right\|_{\hat{B}} \leq \frac{L h^{\alpha} \Gamma(\alpha-n+1)}{\Gamma(2 \alpha-n+1)}\left\|A^{j-1} x-A^{j-1} \tilde{x}\right\|_{\hat{B}},
$$

provando a desigualdade 3.26. Aplicando o Teorema A.2 com $\alpha_{j}=\gamma^{j}$ onde $\gamma=\frac{L h^{\alpha} \Gamma(\alpha-n+1)}{\Gamma(2 \alpha-n+1)}$, devemos mostrar que a série $\sum_{j=0}^{\infty} \alpha_{j}$ converge. Para isso basta notar que $h \leq \tilde{h}$ e da definição de $\tilde{h}, \gamma<1$. Assim mostramos que o teorema do ponto fixo de Weissinger leva à existência e unicidade da solução dada pela integral de Volterra.

Tendo estabelecido as condições de existência e unicidade para as EDO's com derivadas de Riemann-Liouville, veremos agora o problema correspondente para o caso das derivadas serem de Caputo.

Vamos considerar a seguinte equação

$$
{ }_{0}^{\mathrm{C}} D_{t}^{\alpha-k} x(t)=f(x, x(t))
$$

Conforme discutimos anteriormente, as condições iniciais para a equação acima são equivalente às EDO's de ordem inteira, ou seja,

$$
D^{k} x(t)=x_{0}^{(k)}, k=0,1, \ldots, n-1
$$

com $n=\lfloor\alpha\rfloor$. Análogo ao Teorema 3.3 , temos então:

Teorema 3.4 [12] Seja $\alpha>0$ e $n=\lfloor\alpha\rfloor$. Além disso,seja $x_{0}^{(0)}, \ldots, x_{0}^{(n-1)} \in \Re, K>0$, e $h^{*} \dot{z} 0$. Defina

$$
G:=\left\{(t, x): t \in\left[0, h^{*}\right],\left|x-\sum_{k=0}^{n-1} \frac{t^{k} x_{0}^{(k)}}{k !}\right| \leq K\right\},
$$

e seja a função $f: G \rightarrow \Re$ contínua. Também defina $M:=\sup _{(t, z) \in G}|f(t, z)| e$

$$
h=\left\{\begin{array}{c}
h^{*}, \text { se } M=0 \\
\min \left\{h^{*},\left(\frac{K \Gamma(n+1)}{M}\right)^{1 / \alpha}\right\} .
\end{array}\right.
$$

Então existe uma função $x \in C[0, h]$ que resolve o problema de valor inicial 3.28 .

Note que, diferentemente do Teorema 3.3. não colocamos como condição a função $f$ ser Lipschitziana. De fato, devido ao maior uso que daremos ao sistema fracionário com operador de Caputo, mostraremos um critério mais fraco que satisfaz apenas a existência da solução. Para a unicidade, o procedimento será análogo à demonstração feita no Teorema 3.3 , usando o teorema do ponto fixo de Weissinger. Em muitas aplicações na ciência e engenharia, como a aplicação às redes neurais fracionárias por exemplo, podemos ter $0<\alpha<1$. Neste caso, o conjunto $G$ definido no teorema 3.4 é simplesmente o retângulo $G=\left[0, h^{*}\right] \times\left[x_{0}^{(0)}-K, x_{0}^{(0)}+K\right]$.

Lema 3.7 Admita as hipóteses do Teorema 3.4. A função $x \in C[0, h]$ é uma solução do problema de valor inicial dado em (3.28), se e somente se é uma solução da equação integral de Volterra

$$
x(t)=\sum_{k=0}^{n-1} \frac{t^{k}}{k !} x_{0}^{(k)}+\frac{1}{\Gamma(\alpha)} \int_{0}^{x}(t-\tau)^{(n-1)} f(\tau, x(\tau)) d \tau
$$

Demonstração. Seja a função

$$
y(t):=f(t, x(t))
$$


com $y \in C[0 . h]$. Da equação 2.29 e da definição da derivada de Riemann-Liouville, podemos escrever

$$
\begin{aligned}
y(t)=f(t, x(t)) & ={ }^{\mathrm{C}} \mathrm{D}_{t}^{\alpha} x(t)={ }^{\mathrm{RL}} \mathrm{D}_{t}^{\alpha}\left(x(t)-\sum_{k=0}^{n-1} \frac{t^{k}}{k !} x^{(k)}(0)\right) \\
& =D^{n} J_{t}^{n-\alpha}\left(x(t)-\sum_{k=0}^{n-1} \frac{t^{k}}{k !} x^{(k)}(0)\right) .
\end{aligned}
$$

Por simplicidade, definiremos

$$
T=T(t):=\sum_{k=0}^{n-1} \frac{t^{k}}{k !} x^{(k)}(0) .
$$

Aplicando a integral inteira $n$ vezes em ambos os lados desta equação, temos

$$
J_{t}^{n} y(t)=J_{t}^{n-\alpha}(x(t)-T)+q(t) .
$$

O grau de $q(t)$ é no máximo $n-1$. e, sendo $y(t)$ contínua, $J_{t}^{n} y(t)$ tem um zero de ordem $n$ ou mais, ou seja,

$$
\left(J_{t}^{n} y(t)\right)^{(k)}(0)=0 \text { para } k=0,1, \ldots, m-1, \text { e }\left(J_{t}^{n} y(t)\right)^{(m)}(0) \neq 0, m \geq n .
$$

Por construção, $x-T$ tem a mesma propriedade e, consequentemente $J_{t}^{n-\alpha}(x(t)-T)$ também. Como o grau do polinômio $q(t)$ é menor que $n$, seu zero na origem precisa ter ordem menor que $n$, o que implica em $q(t)=0$. Assim,

$$
J_{t}^{n} y(t)=J_{t}^{n-\alpha}(x(t)-T),
$$

e da Equação (2.11), aplicando a derivada de Riemann-Liouville nesta equação, temos

$$
x(t)-T={ }^{\mathrm{RL}} \mathrm{D}_{t}^{n-\alpha} J^{n} y(t)
$$

e, da lei dos expoentes,

$$
x(t)-T=D J_{t}^{1+\alpha-n} J_{t}^{n} y(t)=D J^{1+\alpha} x(t)=J_{t}^{\alpha} y(t) .
$$

Lembrando da definição da integral de Riemann-Liouville e de $T$, chegamos na Equação (3.29) conforme desejado.

Demonstração do teorema 3.4. A demonstração deste Teorema será feita definindo um conjunto $U$ e um operador $A$, satisfazendo as hipóteses do teorema do ponto fixo de Schauder (Apêndice A), a equação integral de Volterra (3.29), que poderá ser escrita como $x=A x$, terá pelo menos um ponto fixo. Para $M=0, f(t, x)=0$ para todo $(t, x) \in G$, e é trivial que a solução 3.29 nestas condições satisfaz o sistema 3.28 (já que a derivada de Caputo de $y$ constante é 0). Vamos definir $T$ como na Equação 3.30 e o conjunto

$$
U:=\left\{x \in C[0, h]:\|x-T\|_{\infty} \leq K\right\}
$$

onde $\|\cdot\|_{\infty}$ é a norma do supremo. Então $U$ é fechado e convexo e é um subconjunto do espaço de funções $C[0, h]$. Para verificar a convexidade de $U$, basta notar que, para $x_{1}, x_{2} \in U$ e $\lambda \in[0,1]$ constante,

$$
\begin{aligned}
\left\|\lambda\left(1-x_{1}\right)+x_{2}-T\right\|_{\infty} & =\left\|\lambda\left(1-x_{1}\right)+x_{2}-T+\lambda T-\lambda T\right\|_{\infty} \\
& =\left\|(1-\lambda) x_{1}+(1-\lambda) T+\lambda\left(x_{2}-T\right)\right\|_{\infty} \\
& \leq(1-\lambda)\left\|x_{1}-T\right\|_{\infty}+\lambda\left\|x_{2}-T\right\|_{\infty} \\
& \leq(1-\lambda) K+\lambda K=K .
\end{aligned}
$$


Como $U$ é um subconjunto do espaço normado completo (espaço de Banach) formado pelas funções $C[0, h], U$ é um espaço de Banach também. Sendo $T$ um elemento de $U$, temos que $U$ não é um espaço vazio. Definindo o operador

$$
(A x)(t):=T+\frac{1}{\Gamma(\alpha)} \int_{0}^{t}(t-\tau)^{\alpha-1} f(\tau, x(\tau)) d \tau
$$

e usando este operador podemos reescrever a equação integral de Volterra (3.29) como

$$
x=A x .
$$

Precisamos agora mostrar que $A$ tem ao menos um ponto fixo. Primeiramente, vamos mostrar que $A x \in U$ para $x \in U$. Para $0 \leq t_{1} \leq t_{2} \leq h$, temos que

$$
\begin{aligned}
& \left|(A x)\left(t_{1}\right)-\left(A x_{2}\right)\left(t_{2}\right)\right| \\
& =\frac{1}{\Gamma(\alpha)}\left|\int_{0}^{t_{1}}\left(t_{1}-\tau\right)^{\alpha-1} f(\tau, x(\tau)) d \tau-\int_{0}^{t_{2}}\left(t_{2}-\tau\right)^{\alpha-1} f(\tau, x(\tau)) d \tau\right| \\
& =\frac{1}{\Gamma(\alpha)}\left|\int_{0}^{t_{1}}\left(\left(t_{1}-\tau\right)^{\alpha-1}-\left(t_{2}-\tau\right)^{\alpha-1}\right)(\tau, x(\tau)) d \tau-\int_{t_{1}}^{t_{2}}\left(t_{2}-\tau\right)^{\alpha-1} f(\tau, x(\tau)) d \tau\right| \\
& \leq \frac{M}{\Gamma(\alpha)}\left(\int_{0}^{t_{1}}\left|\left(t_{1}-\tau\right)^{\alpha-1}-\left(t_{2}-\tau\right)^{\alpha-1}\right| d \tau+\int_{t_{1}}^{t_{2}}\left(t_{2}-\tau\right)^{\alpha-1} d \tau\right) \\
& \left|(A x)\left(t_{1}\right)-\left(A x_{2}\right)\left(t_{2}\right)\right| \leq \frac{M}{\Gamma(\alpha)}\left(\int_{0}^{t_{1}}\left|\left(t_{1}-\tau\right)^{\alpha-1}-\left(t_{2}-\tau\right)^{\alpha-1}\right|+\frac{\left(t_{2}-t_{1}\right)^{\alpha}}{\alpha}\right) .
\end{aligned}
$$

Para resolver a integral na Equação (3.32), consideraremos três casos: $\alpha=1, \alpha<1$ e $\alpha \geq 1$. No caso em que $\alpha=1$, a integral é nula. Para $\alpha<1, \alpha-1<0$ e $\left(t_{1}-\tau\right)^{\alpha-1} \geq\left(t_{2}-\tau\right)^{\alpha-1}$, o que leva a

$$
\begin{aligned}
\int_{0}^{t_{1}}\left|\left(t_{1}-\tau\right)^{\alpha-1}-\left(t_{2}-\tau\right)^{\alpha-1}\right| d \tau & =\int_{0}^{t_{1}}\left(\left(t_{1}-\tau\right)^{\alpha-1}-\left(t_{2}-\tau\right)^{\alpha-1}\right) d \tau \\
& =\frac{1}{\alpha}\left(t_{1}^{\alpha}+t_{2}^{\alpha}+\left(t_{1}+t_{2}\right)^{\alpha}\right) \leq \frac{1}{\alpha}\left(t_{2}-t_{1}\right)^{\alpha}
\end{aligned}
$$

Para $\alpha>1, \alpha-1>0$ e $\left(t_{1}-\tau\right)^{\alpha-1} \leq\left(t_{2}-\tau\right)^{\alpha-1}$, e daí

$$
\begin{aligned}
\int_{0}^{t_{1}}\left|\left(t_{1}-\tau\right)^{\alpha-1}-\left(t_{2}-\tau\right)^{\alpha-1}\right| d \tau & =\int_{0}^{t_{1}}\left(\left(t_{2}-\tau\right)^{\alpha-1}-\left(t_{1}-\tau\right)^{\alpha-1}\right) d \tau \\
& =\frac{1}{\alpha}\left(\left(t_{2}^{\alpha}-t_{1}^{\alpha}+\left(t_{2}-t_{1}\right)^{\alpha}\right) \leq \frac{1}{\alpha}\left(t_{2}^{\alpha}-t_{1}^{\alpha}\right) .\right.
\end{aligned}
$$

A partir destes resultados temos que a expressão. 3.32 pode ser escrita como

$$
\left\|(A x)\left(t_{1}\right)-(A x)\left(t_{2}\right)\right\| \leq\left\{\begin{array}{c}
\frac{2 M}{\Gamma(2 \alpha+1)}\left(t_{2}-t_{1}\right)^{\alpha} \text { se } \alpha \leq 1 \\
\frac{M}{\Gamma(2 \alpha+1)}\left(\left(t_{2}-t_{1}\right)^{\alpha}+t_{2}^{\alpha}-t_{1}^{\alpha}\right) \text { se } \alpha>1
\end{array} .\right.
$$

É claro que o lado direito de 3.33 converge para zero quando $t_{2} \rightarrow t_{1}$, mostrando que $A x$ é contínua. Além disso, da definição de $h$ e sendo $y \in U, t \in[0, h]$, temos

$$
\begin{aligned}
|(A x)(t)-T| & =\frac{1}{\Gamma(\alpha)}\left|\int_{0}^{t}(t-\tau)^{\alpha-1} f(\tau, x(\tau)) d \tau\right| \\
& \leq \frac{1}{\Gamma(\alpha+1)} M t^{\alpha} \leq \frac{1}{\Gamma(\alpha+1)} M h^{\alpha} \leq \frac{1}{\Gamma(\alpha+1)} \frac{M K \Gamma(\alpha+1)}{M}=K,
\end{aligned}
$$


o que mostra que se $x \in U, A x \in U$.

Agora, para satisfazer as hipóteses do teorema do ponto fixo de Schauder, resta apenas mostrar que $A(U):\{A u: u \in U\}$ é relativamente compacto, ou seja, que o fecho de $A u$ é compacto em $U$. Como estamos em um espaço de funções com dimensão infinita, não necessariamente um conjunto fechado e limitado, é compacto. Para mostrarmos que um conjunto é relativamente compacto, a maneira mais natural é aplicarmos o teorema de Arzelà-Ascóli (Apêndice A). Para $y \in A(U)$, temos que

$$
\begin{aligned}
|z(t)| & =|(A x)(t)|=\left|T+\frac{1}{\Gamma(\alpha)} \int_{0}^{t}(t-\tau)^{\alpha-1} f(\tau, x(\tau)) d \tau\right| \\
& \leq|T|+\frac{1}{\Gamma(\alpha)} \int_{0}^{t}(t-\tau)^{\alpha-1}|f(\tau, x(\tau))| d \tau \\
& \leq\|T\|_{\infty}+\frac{1}{\Gamma(\alpha+1)} M h^{\alpha} \leq\|T\|_{\infty}+K \leq C,
\end{aligned}
$$

o que mostra que $z$ é uniformemente limitada, pois $\|z(t)\|_{\infty} \leq C$ constante. A equicontinuidade pode ser derivada de (3.33). Para $0 \leq t_{1} \leq t_{2} \leq h$, devemos mostrar que para $\epsilon>0$, existe $\delta>0$ tal que para todo $z \in U,\left|t_{1}-t_{2}\right|<\delta$ implica $\left|x\left(t_{1}\right)-x\left(t_{2}\right)\right|<\epsilon$ para $n \leq 1$ obtemos

$$
\left|\left(A x\left(t_{1}\right)\right)-\left(A x\left(t_{2}\right)\right)\right| \leq \frac{2 M}{\Gamma(\alpha+1)}\left(t_{2}-t_{1}\right)^{\alpha}=\frac{2 M}{\Gamma(\alpha+1)} \delta^{\alpha},
$$

onde basta escolher $\delta=\left(\frac{\Gamma(\alpha+1)}{2 M} \epsilon\right)^{1 / \alpha}$. Note que $\delta$ não depende de $x, t_{1}$ e $t_{2}$, mostrando assim que o conjunto $A(U)$ é equicontínuo Para $n>1$, usando o teorema do valor médio concluímos que

$$
\begin{aligned}
\left|\left(A x\left(t_{1}\right)\right)-\left(A x\left(t_{2}\right)\right)\right| & \left.\leq \frac{M}{\Gamma(\alpha+1)}\left(\left(t_{2}-t_{1}\right)^{\alpha}+t_{2}^{\alpha}-t_{1}^{\alpha}\right)\right) \\
& =\frac{M}{\Gamma(\alpha+1)}\left(\left(t_{2}-t_{1}\right)^{\alpha}+\alpha\left(t_{2}-t_{1}\right) t_{3}^{\alpha-1}\right) \\
& \leq \Gamma(\alpha+1)\left(\delta^{\alpha}+\alpha \delta h^{\alpha-1}\right)
\end{aligned}
$$

para algum $t_{3} \in\left[t_{1}, t_{2}\right] \in[0, h]$. Como o lado direito da expressão acima é independente de $x, t_{1}$ e $t_{2}$ provamos que para $\alpha>1$, o conjunto $A(U)$ também é equicontínuo. Assim, do teorema de ArzeláAscóli leva à conclusão de que $A(U)$ é relativamente compacto e, dado que $A(U)$ é uniformemente limitado, podemos finalmente aplicar o teorema do ponto fixo de Schauder, demonstrando que $A$ tem ao menos um ponto fixo.

Veja que o problema de continuidade de $x$ que tínhamos com o problema de valor inicial com a derivada de Riemann-Liouville não existe para o caso em que a derivada é de Caputo, por isso temos que a continuidade de $f$ implica continuidade da solução $x$ através do intervalo fechado $[0, h]$.

Teorema 3.5 Seja $0<\alpha$ e $n$ o menor inteiro maior que $\alpha$. Além disso, seja $x_{0}^{(0)}, \ldots x_{0}^{(n-1)} \in$ $\Re, K>0$ e $h^{*}>0$. Defina $G$ como no Teorema 3.4 e $f: G \rightarrow \Re$ contínua e satisfazendo a condição de Lipschitz com respeito à segunda variável, ou seja,

$$
\left|f\left(t, x_{1}\right)-f\left(t, x_{2}\right)\right| \leq L\left|t_{1}-t_{2}\right|
$$

com $L>0$ constante. Então existe uma única solução definida em $h \in[0, h]$ resolvendo o problema de valor inicial (3.28).

Demonstração. A demonstração é análoga ao teorema 3.3 onde usamos o teorema do ponto fixo de Weissinger para demonstrar a unicidade da solução. Seja o polinômio $T$ definido em (3.30), o operador $A$ definido em (3.31) e o conjunto $U$ definido em (3.5), equipado com a norma do supremo. Para provar que $A$ tem um único ponto fixo, temos que verificar que, para cada $j \in \mathbf{N}$, 
cada $t \in[0, h]$ e $x, \tilde{x} \in U$,

$$
\left\|A^{j} x-A^{j} \tilde{x}\right\|_{\infty} \leq \frac{\left(L t^{\alpha}\right)^{j}}{\Gamma(1+\alpha j)}\|x-\tilde{x}\|_{\infty}
$$

Por indução, quando $j=0$ é trivial. Para $j \rightarrow j+1$, temos

$$
\begin{aligned}
& \left\|A^{j+1} x-A^{j+1} \tilde{x}\right\|_{\infty}=\left\|A\left(A^{j} x\right)-A\left(A^{j} \tilde{x}\right)\right\|_{\infty} \\
& =\frac{1}{\Gamma(\alpha)} \sup _{0 \leq w \leq h}\left|\int_{0}^{w}(w-\tau)^{\alpha-1}\left[f\left(t, A^{j} x(\tau)\right)-f\left(t, A^{j} \tilde{x}(\tau)\right)\right] d \tau\right| \\
& \leq \frac{L}{\Gamma(\alpha)} \sup _{0 \leq w \leq h} \int_{0}^{w}(w-\tau)^{\alpha-1}\left|A^{j} x(\tau)-A^{j} \tilde{x}(\tau)\right| d \tau,
\end{aligned}
$$

devido à condição de Lipschitz de $f$. Aplicando a hipótese indutiva, temos que

$$
\begin{aligned}
& \left\|A^{j+1} x-A^{j+1} \tilde{x}\right\|_{\infty} \\
& \leq \frac{L}{\Gamma(\alpha)} \int_{0}^{h}(h-\tau)^{\alpha-1} \sup _{0 \leq w \leq h}\left|A^{j} x(w)-A^{j} \tilde{x}(w)\right| d \tau \\
& \leq \frac{L}{\Gamma(\alpha)} \int_{0}^{h}(h-\tau)^{\alpha-1} \frac{\left(L t^{\alpha}\right)^{j}}{\Gamma(1+\alpha j)}\|x-\tilde{x}\|_{\infty} d \tau \\
& \leq \frac{L^{j+1}}{\Gamma(\alpha) \Gamma(1+\alpha j)} \int_{0}^{h}(h-\tau)^{\alpha-1} h^{\alpha j} d \tau\|x-\tilde{x}\|_{\infty} d \tau \\
& \leq \frac{L^{j+1}}{\Gamma(1+\alpha j)} J_{h}^{\alpha} h^{\alpha j} \sup _{0 \leq w \leq h}|x-\tilde{x}|,
\end{aligned}
$$

e do Exemplo 2.1, chegamos em

$$
\begin{aligned}
\left\|A^{j+1} x-A^{j+1} \tilde{x}\right\|_{\infty} & \leq \frac{L^{j+1}}{\Gamma(1+\alpha j)} \frac{\Gamma(1+\alpha j) h^{\alpha(j+1)}}{\Gamma(\alpha(j+1)+1)}\|x-\tilde{x}\|_{\infty} \\
\left\|A^{j+1} x-A^{j+1} \tilde{x}\right\|_{\infty} & \leq \frac{\left(L h^{\alpha}\right)^{j+1}}{\Gamma(\alpha(j+1)+1)}\|x-\tilde{x}\|_{\infty},
\end{aligned}
$$

que é o resultado que esperávamos. Para aplicarmos o teorema do ponto fixo de Weissinger, é ainda necessário verificar que $\sum_{j=0}^{\infty} \gamma_{j}$, com $\gamma_{j}=\frac{\left(L t^{\alpha}\right)^{j}}{\Gamma(1+\alpha j)}$ converge. Mas note que essa é a função de Mittag-Leffler, ou seja, $\sum_{j=0}^{\infty} \gamma_{j}=E_{\alpha}\left(L t^{\alpha}\right)$, que do Lema 3.1 sabemos que converge. Daí segue que o operador $A$ tem apenas um ponto fixo.

Exemplo 3.1 Considere a seguinte equação:

$$
{ }_{0}^{\mathrm{RL}} \mathrm{D}_{t}^{\alpha} x(t)=(x(t))^{\mu}
$$

onde $0<\mu<1$. Observe que o lado direito desta equação viola a condição de Lipschitz, pois

$$
\frac{\left|x^{\mu}-y^{\mu}\right|}{|x-y|}
$$

quando $y=0$ fica ilimitado para qualquer constante quando $t \rightarrow \infty$. Com as condições iniciais

$$
{ }_{0}^{\mathrm{RL}} \mathrm{D}_{t}^{\alpha-k} x(0)=0(k=1,2, \ldots, m-1), \quad \lim _{z \rightarrow 0^{+}} J^{m-\alpha} x(z)=0,
$$

podemos ver que uma solução trivial é $x \equiv 0$. Mas um cálculo explícito nos revela também que

$$
\left(\frac{\Gamma(j+1)}{\Gamma(j+1-n)}\right)^{\frac{1}{\mu-1}} x^{1 / j},
$$


com $j=n /(1-\mu)$, também resolve o problema de valor inicial dado, o que mostra que nem sempre a unicidade é garantida quando a condição de Lipschitz não é válida.

O mesmo acontece com a EDOF com o operador de Caputo

$$
{ }_{0}^{\mathrm{C}} \mathrm{D}_{t}^{\alpha} y(t)=(y(t))^{\mu}
$$

onde $0<\mu<1$. Supondo que as condições iniciais para essa equação são

$$
y(0)=0 \text { e } D^{\alpha-k} y(0)=0,(k=1,2, \ldots, n-1)
$$

que leva às mesmas soluçôes para o caso em que a EDOF dada tem o operador de RiemannLiouville.

Um dos problemas com o Teorema 3.4 é que a solução é garantida apenas no intervalo $[0, h]$. Em algumas situações é importante saber em quais condições a solução de uma EDOF existe para o intervalo $[0, \infty)$. Portanto, o seguinte resultado pode ser útil:

Teorema 3.6 Admita as hipóteses do Teorema 3.4 exceto que o conjunto $G$, que é dominio da função $f$ definida no lado direito de (3.28), é agora tomada como $G:=\mathcal{R}^{2}$. Então existe uma função $x \in C[0, \infty]$ que resolve o problema de valor inicial (3.28).

Demonstração: Suponha que existem constantes $c_{1} \geq 0, c_{2} \geq 0$ e $0 \leq \mu \leq 1$ tal que

$$
|f(t, x)| \leq c_{1}+c_{2}|x|^{\mu}
$$

para todo $(t, x) \in G$. Usando a função polinomial $T$ definida em 3.30 , temos que, para $\mu<1$, nós podemos encontrar algum $K>0$ tal que

$$
c_{1}+c_{2}\left(K+\max _{x \in\left[0, h^{*}\right]}|T(x)|\right)^{\mu} \leq \frac{K \Gamma(\alpha+1)}{h^{* \alpha}} .
$$

Usando este valor de $K$, nós então restringimos a função $f$ para o conjunto $G_{k}:=(t, x): x \in\left[0, h^{*}\right],[x-T] \leq K$. Então temos que

$$
\begin{aligned}
M & :=\sup _{(t, x) \in G_{k}}|f(t, x)| \leq c_{1}+c_{2} \sup _{(t, x) \in G_{k}}|x|^{\mu} \\
& \leq c_{1}+c_{2}\left(K+\max _{x \in\left[0, h^{*}\right]}|T(x)|\right)^{\mu} \leq \frac{K \Gamma(\alpha+1)}{h^{* \alpha}} .
\end{aligned}
$$

Para este valor de $K$ podemos ver da definição de $h$ no teorema 3.4 que $\left(\frac{K \Gamma(\alpha+1)}{M}\right)^{1 / \alpha} \geq h^{*}$ o que implica em

$$
h^{*}=\min \left\{h^{*},\left(\frac{K \Gamma(\alpha+1)}{M}\right)^{1 / \alpha}\right\}=h .
$$

Assim, mostramos que a função $x \in C\left(\left[0, h^{*}\right]\right)$ e a função $G$ pode ser tomada em $G=\left[0, h^{*}\right] \times \mathcal{R}$. Como podemos escolher $h^{*}$ arbitrariamente grande, então temos que existe uma solução contínua no intervalo $[0, \infty]$ se tomarmos $G=\mathcal{R}^{2}$.

\subsection{Estabilidade de Lyapunov}

Nesta seção, vamos apresentar alguns conceitos fundamentais que embasam a análise de estabilidade para equações diferenciais ordinárias e introduzir a estabilidade no sentido de Lyapunov. 
Definição 3.5 (Ponto de equilíbrio) Considere a seguinte sistema não-autônomo de equações diferenciais ordinárias

$$
\begin{aligned}
& \dot{x}=f(t, x(t)) \\
& x\left(t_{0}\right)=x_{0}
\end{aligned}
$$

com $f: D \rightarrow \Re^{n}, D \subset \Re^{n}$ aberto e $f$ satisfazendo as condições usuais do teorema de Picard de existência e unicidade de soluções. Então $\bar{x}$ é denominado ponto de equilíbrio de do sistema 3.35 se $f(t, \bar{x})=0$.

Nosso objetivo é saber se as trajetórias próximas ao ponto de equilíbrio $\bar{x}$ são "bem comportadas". Vamos definir agora a noção de estabilidade e atratividade do ponto de equilíbrio. As definições de estabilidade dadas a seguir também podem ser estendidas para EDO's fracionárias.

Definição 3.6 (Estabilidade e Atratividade) O ponto de equilíbrio $\bar{x}$ do sistema (3.35) é denominado estável se

$$
\begin{gathered}
\forall \epsilon>0, \exists \delta(\epsilon)>0 \\
\|x(0)-\bar{x}\|<\delta \Rightarrow\|x(t)-\bar{x}\|<\epsilon, \forall t \geqslant t_{0} ;
\end{gathered}
$$

e denominado atrativo quando $\exists \delta>0$ tal que

$$
\|x(0)-\bar{x}\|<\delta \Rightarrow \lim _{t \rightarrow \infty} x(t)=\bar{x} .
$$

É denominado assintoticamente estável quando é simultaneamente estável e atrativo.

A ideia desta definição é mostrar que se a trajetória inicia-se numa bola aberta de centro $\bar{x}$ e raio $\delta$, ela sempre irá permanecer dentro de uma bola aberta de centro $\bar{x}$ e raio $\epsilon$ que depende de $\delta$. Para o caso de atratividade, a trajetória irá convergir ao ponto de equilíbrio $\bar{x}$. As Figuras $3.2 \mathrm{e}$ 3.3 ilustram bem as ideias apresentadas para o caso bidimensional.

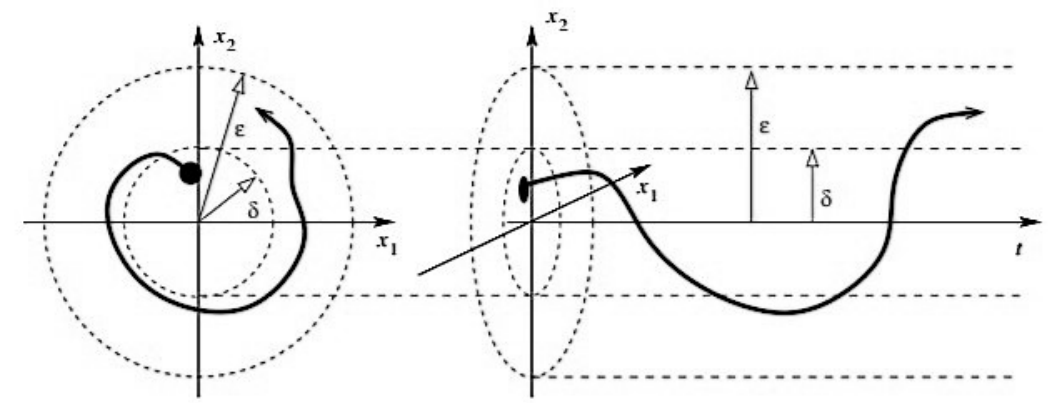

Figura 3.2: Noção geométrica de estabilidade em duas dimensões.

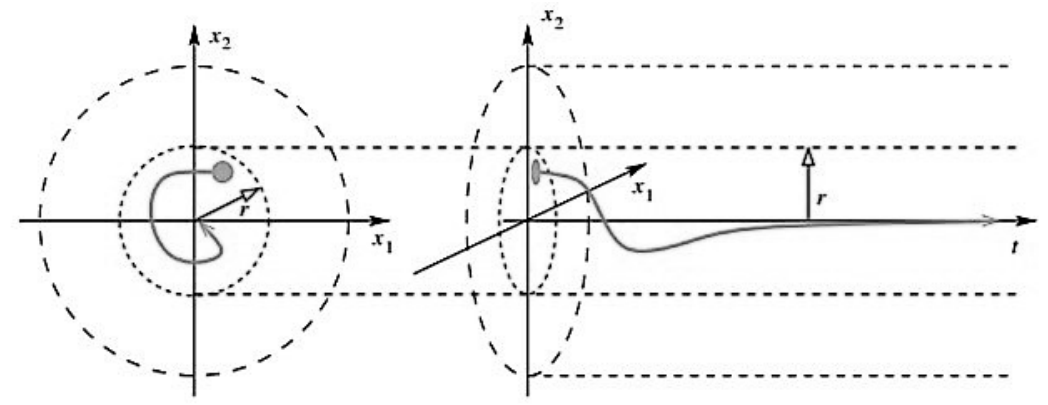

Figura 3.3: Noção geométrica de estabilidade assintótica em duas dimensões.

Há ainda outros conceitos importantes sobre estabilidade que precisam ser definidos. 
Definição 3.7 (Estabilidade Exponencial) O ponto de equilíbrio $\bar{x}$ do sistema (3.35) é exponencialmente estável se existem constantes positivas $c, k$ e $\lambda$ tais que

$$
\|x(t)\| \leq k\left\|x\left(t_{0}\right)\right\| e^{-\lambda\left(t-t_{0}\right)}
$$

para todo $\left\|x\left(t_{0}\right)\right\|<c$. É globalmente exponencialmente estável quando a desigualdade é satisfeita para todo $\left\|x\left(t_{0}\right)\right\|$.

O método direto de Lyapunov para o cálculo fracionário que será estudado mais adiante foi inspirado no seguinte teorema que caracteriza a estabilidade exponencial:

Teorema 3.7 Seja $x=0$ o ponto de equilíbrio para o sistema não autônomo $\dot{x}=f(t, x)$ que pertence a um subconjunto $D \in \Re^{n}$. Seja $V:[0, \infty) \times D \rightarrow \Re$ uma função continuamente diferenciável tal que

$$
\begin{aligned}
& k_{1}\|x\|^{a} \leq V(t, x(t)) \leq k_{2}\|x\|^{a} \\
& \frac{\partial V}{\partial t}+\frac{\partial V}{\partial x} f(t, x) \leq-k_{3}\|x\|^{a},
\end{aligned}
$$

para todo $t \geq 0$ e $x \in D$, onde $k_{1}, k_{2}, k_{3}$ e a são constantes positivas. Então $x=0$ é exponencialmente estável. Caso a suposição seja assegurada globalmente, $x=0$ é globalmente exponencialmente estável.

Demonstração. A partir das equações (3.37) e (3.38), temos que

$$
\dot{V} \leq-\frac{k_{3}}{k_{2}} V
$$

o que leva a

$$
V(t, x(t)) \leq V\left(t_{0}, x\left(t_{0}\right)\right) e^{-\frac{k_{3}}{k_{2}}\left(t-t_{0}\right)}
$$

Consequentemente,

$$
\begin{aligned}
\|x\| \leq\left[\frac{V(t, x(t))}{k_{1}}\right]^{\frac{1}{a}} & \leq\left[\frac{V\left(t_{0}, x\left(t_{0}\right)\right) e^{-\frac{k_{3}}{k_{2}}\left(t-t_{0}\right)}}{k_{1}}\right]^{\frac{1}{a}} \\
& \leq\left[\frac{k_{2}\left\|x\left(t_{0}\right)\right\|^{a} e^{-\frac{k_{3}}{k_{2}}\left(t-t_{0}\right)}}{k_{1}}\right]^{\frac{1}{a}} \\
& \leq\left(\frac{k_{2}}{k_{1}}\right)^{a}\left\|x\left(t_{0}\right)\right\| e^{-\frac{k_{3}}{k_{2}}\left(t-t_{0}\right)}
\end{aligned}
$$

e a estabilidade exponencial decorre de 3.36 , fazendo $k=\left(\frac{k_{2}}{k_{1}}\right)^{a}$ e $\lambda=\frac{k_{3}}{k_{2}}$.

A noção de estabilidade uniforme é particularmente importante na análise de estabilidade de sistemas não autônomos. Para os sistemas autônomos, em que não há dependência explícita com o tempo, as propriedades de estabilidade são automaticamente uniformes em relação ao instante inicial $t_{0}$.

Definição 3.8 (Estabilidade Uniforme) O ponto de equilíbrio $\bar{x}$ do sistema (3.35) é uniformemente estável quando é estável e o raio $\delta=\delta\left(t_{0}\right)$ não depende do instante $t_{0}$ considerado. De forma análoga, um ponto de equilíbrio é uniformemente assintoticamente estável quando é assintoticamente estável e o raio $\delta=\delta\left(t_{0}\right)$ não depende do instante $t_{0}$.

Também precisamos ter condições que caracterizem quando o sistema é globalmente estável.ou seja, estável para todo $\mathbb{R}^{n}$. 
Definição 3.9 (Estabilidade Assintótica Global) O ponto de equilíbrio $\bar{x}$ é assintoticamente globalmente estável quando as condições de estabilidade não valem apenas próximo ao ponto de equilíbrio, mas para todo o espaço $\mathbb{R}^{n}$, ou seja, para todo $\bar{x}\left(t_{0}\right) \in \Re^{n},\|\bar{x}(t)\| \rightarrow 0$ quando $t \rightarrow \infty$.

\subsubsection{Método de Lyapunov}

A. M. Lyapunov [37] desenvolveu métodos para verificar a estabilidade de sistemas dinâmicos não lineares. O método direto de Lyapunov, chamado também de segundo método de Lyapunov é um método eficiente de verificar a estabilidade. Dado um sistema não linear da forma $\dot{x}=f(x)$, é obtida uma determinada função de Lyapunov que nos fornecerá informações sobre a estabilidade do ponto de equilíbrio do sistema.

Definição 3.10 (Função de Lyapunov) Uma função $V: U \rightarrow \Re$, onde $U$ é uma vizinhança qualquer de $\bar{x}=0$, é denominada função de Lyapunov para $\dot{x}=f(t, x(t))$ quando satisfaz as seguintes condições:

1. $V$ é contínua em $U$ e continuamente diferenciável em $U \backslash\{0\}$;

2. $V(0)=0 ; V(x)>0, \forall x \in U, x \neq 0$;

3. $\dot{V}(x)=\langle\nabla V(x), f(t, x(t))\rangle=\nabla V(x)^{*} f(t, x(t)) \leq 0, \forall x \in U \backslash\{0\}$.

Teorema 3.8 (Método Direto de Lyapunov) Seja $U$ uma vizinhança qualquer de $\bar{x}=0$ e $V: U \rightarrow \Re$ uma função de Lyapunov para o sistema $\dot{x}=f(t, x(t))$. Então, $\bar{x}=0$ é um ponto de equilíbrio estável.

Demonstração: Vamos escolher $r>0$ e definir

$$
B_{r}=\left\{x \in \Re^{n}:\|x\| \leq r\right\} \subset D .
$$

$B_{r}$ é um conjunto fechado e limitado (compacto, uma esfera). Construiremos uma superfície de Lyapunov em $B_{r}$ e mostraremos que toda trajetória iniciando próximo de $\bar{x}=0$ permanece nesta superfície (Definição 3.6). Seja

$$
\alpha=\min _{\|x\| \rightarrow r} V(x) \quad \alpha>0
$$

Escolha $\beta \in(0, \alpha)$ e denote

$$
\Omega_{\beta}=\left\{x \in B_{r}: V(x) \leq \beta\right\} .
$$

Temos, por construção, $\Omega_{\beta} \subset B_{r}$. Agora admita que $x(0) \in \Omega_{\beta}$. Daí:

$$
\dot{V}(x) \leq 0 \Rightarrow V(x) \leq V(x(0)) \leq \beta, \quad \forall t \geq 0 .
$$

Portanto, as trajetórias que se iniciam em $\Omega_{\beta}$ em $t=0$ permanecem em $\Omega_{\beta}$ para todo $t \geq 0$. Pela continuidade de $V(x)$, existe $\delta>0$ tal que

$$
\|x\|<\delta \Rightarrow V(x)<\beta,
$$

ou seja, $B_{\delta} \subset \Omega_{\beta} \subset B_{r}$. Em seguida temos que

$$
\|x(0)\|<\delta \Rightarrow x(t) \in \Omega_{\beta} \subset B_{r}, \quad \forall t>0
$$

e daí

$$
\|x(0)\|<\delta \Rightarrow\|x(t)\|<r \leq \epsilon, \quad \forall t \geq 0
$$

o que mostra que o ponto de equilíbrio $\bar{x}=0$ é estável.

Uma observação importante é que Se a função de Lyapunov satisfizer a condição $\langle\nabla V(x), f(t, x(t))\rangle<$ 0 , a função de Lyapunov é estrita e o ponto de equilíbrio do sistema $\dot{x}=f(t, x(t))$ é assintoticamente estável. 
Uma interpretação física para o método direto de Lyapunov é considerar $V(x)$ como função de energia do sistema, Se a energia total de um sistema é dissipada continuamente, então este sistema deve se acomodar em um ponto de equilíbrio. Neste caso, a analogia que se faz é que função de energia $V(x)$ é sempre positiva, e sua derivada temporal ao longo das trajetórias, ${ }^{'} \dot{V}(x)<0$, representa a dissipação desta energia. [54]

Exemplo 3.2 (Circuito elétrico) Considere a equação diferencial ordinária associada ao circuito elétrico $R L C$ :

$$
C L \ddot{x}+R C \dot{x}+x=0,
$$

temos como sistema associado

$$
\left[\begin{array}{c}
\dot{x_{1}} \\
\dot{x_{2}}
\end{array}\right]=\left[\begin{array}{cc}
0 & 1 \\
-(L C)^{-1} & -R L^{-1}
\end{array}\right]\left[\begin{array}{l}
x_{1} \\
x_{2}
\end{array}\right],
$$

com $R, L, C>0$. Definimos uma função de Lyapunov para este sistema dada por

$$
V\left(x_{1}, x_{2}\right):=L x_{2}^{2}+C^{-1} x_{1}^{2},\left(x_{1}, x_{2}\right) \in \Re^{2},
$$

de onde calculamos

$$
\left\langle\nabla V\left(x_{1}, x_{2}\right), f\left(x_{1}, x_{2}\right)\right\rangle=\left[\begin{array}{ll}
2 C^{-1} x_{1} & 2 L x_{2}
\end{array}\right]\left[\begin{array}{c}
x_{2} \\
-(L C)^{-1} x_{1}-R L^{-1} x_{2}
\end{array}\right]=-2 R x_{2}^{2},
$$

$\operatorname{com}\left(x_{1}, x_{2}\right) \in \Re^{2}$.O Teorema 3.12 garante que $\bar{x}=0$ é estável.

\subsubsection{Princípio da Invariância de LaSalle}

O princípio de invariância de LaSalle, desenvolvido por LaSalle em 1960 [31], estabelece um critério para estabilidade assintótica dos pontos de equilíbrio por meio do conjunto $\omega$-limite das soluções de um sistema não linear autônomo. Suponha o seguinte sistema

$$
\dot{x}=f(x(t)),
$$

com $x\left(t_{0}\right)=x_{0}$. Seja $\gamma\left(x_{0}\right)=\left\{x\left(t, t_{0} ; x_{0}, t \geq 0\right\}\right.$ a órbita positiva de 3.39 iniciando em $x_{0}$. O conjunto invariante

$$
\omega\left(x_{0}\right)=\left\{x \in \mathcal{R}^{n}: \exists t_{n} \rightarrow+\infty \mid x=\lim _{n \rightarrow+\infty} x\left(t_{n}, t_{0} ; x_{0}\right)\right\}
$$

é o $\omega$-limite das órbitas por $x_{0}$, que pode ser interpretado como o conjunto em que a órbita positiva $\gamma$ que passa por $x_{0}$ se acumula decorrido uma quantidade suficientemente grande de tempo. Então temos o seguinte teorema:

Teorema 3.9 (Princípio da Invariância de LaSalle) Seja $V: \mathcal{R}^{n} \rightarrow \mathcal{R}$ uma função de classe $C^{1}$ tal que $\dot{V}(x(t)) \leq 0$. Então temos que

$$
\omega\left(x_{0}\right) \subset E=\left\{x \in \mathcal{R}^{n}: \dot{V}(x(t))=0 .\right\}
$$

Demonstração [47]. Suponha que $\omega\left(x_{0}\right)$ não divirja. Como $\dot{V} \leq 0, V\left(x\left(t, x_{0}\right)\right)$ é decrescente. Se $y \in \omega\left(x_{0}\right)$, então existem $t_{n} \rightarrow \infty$ tais que $x\left(t_{n}, x_{0}\right) \rightarrow y$. E, pela continuidade de $V, V\left(t_{n}\right) \rightarrow V(y)$. .Como a função $V$ é monotônica, $V(t) \rightarrow c=V(y)$, quando $t \rightarrow \infty$, onde $c$ é uma constante. Assim, como o conjunto $\omega$-limite é invariante, isto é, se $y \in \omega\left(x_{0}\right)$ então $x(t, y) \in \omega\left(x_{0}\right)$ para todo $t$, temos que $V(x(t, y))=c$ e, como consequência, $\dot{V}=0$. Então $y \in E$, como queríamos demonstrar.

Exemplo 3.3 Considere a equação do pêndulo amortecido

$$
\left\{\begin{array}{l}
\dot{x}_{1}=x_{2} \\
\dot{x}_{2}=-\frac{g}{l} \operatorname{sen}\left(x_{1}\right)-\frac{k}{m} x_{2}
\end{array}\right.
$$


onde $g$ é a aceleração da grvidade, $l$ o comprimento do fio, $k$ a constante de amortecimento e $m$ a massa da esfera. Considere a seguinte função candidata de Lyapunov

$$
V(x)=\frac{g}{l}\left(1-\cos \left(x_{1}\right)\right)+\frac{1}{2} x_{2}^{2} .
$$

Note que

$$
\begin{aligned}
\dot{V}(x) & =\frac{g}{l} \dot{x}_{1} \operatorname{sen}\left(x_{1}\right)+x_{2} \dot{x}_{2} \\
\dot{V}(x) & =-\frac{k}{m} x_{2}^{2},
\end{aligned}
$$

portanto $\dot{V}(x) \leq 0$. Do princípio da invariância de LaSalle, temos que o conjunto $E$ é dado por $E=\left\{\left(x_{1}, x_{2}\right) \in \mathbb{R}: x_{2}=0\right\}$ e, como $x_{2}=0$, temos que $\dot{x}_{2}=0$, o que implica em $x_{1}=0$, pois $\frac{g}{l} \operatorname{sen}\left(x_{1}(t)\right)=0$. Então, o conjunto $E$ contém apenas a solução $x^{*}=0$, e com isso temos que o conjunto $\omega$-limite de qualquer solução do sistema é $\{0,0\}$.

\subsection{Estabilidade de Mittag-Leffler}

Tendo visto os fundamentos das equações diferenciais em ordem fracionária, prosseguiremos com o estudo da estabilidade deste tipo de sistema. Como vimos, em sistemas não lineares, o método direto de Lyapunov (ou segundo método de Lyapunov), provê uma maneira de analisar a estabilidade de um sistema sem necessariamente resolvê-lo. Uma observação importante, é que em sistemas de ordem fracionária as soluções não satisfazem a propriedade clássica de fluxo de sistemas dinâmicos [32]. Portanto, não podemos usar esse argumento para a demonstração dos resultados de estabilidade como são provados no contexto de equações diferenciais ordinárias.

\subsubsection{Conceito de ponto de equilíbrio para sistemas de ordem fracionária}

A definição de ponto de equilíbrio para sistemas em ordem fracionária é a mesma para sistemas de ordem inteira. Considere o seguinte sistema de Riemann-Liouville

$$
\left\{\begin{array}{c}
{ }_{\mathrm{t}_{0}} \mathrm{D}_{t}^{\alpha} x(t)=f(t, x(t)) \\
x\left(t_{0}\right)=x_{t_{0}}
\end{array}\right.
$$

onde $\alpha \in(0,1), x=\left(x_{1}, x_{2}, \ldots, x_{n}\right)^{T} \in \Re^{n}, t_{0} \geqslant 0$ o tempo inicial e $f:(0,+\infty] \times \Re^{n} \rightarrow \Re^{n}$ contínua por partes em $t$ satisfazendo a condição de Lipschitz local em $x$.

Definição 3.11 A constante $\tilde{x}$ é um ponto de equilíbrio do Sistema 3.41 se e somente se $f(t, \tilde{x})=$ 0 , $\tilde{x}$ é solução, ou seja, ${ }_{\mathrm{t}_{0}}^{\mathrm{RL}} \mathrm{D}_{t}^{\alpha} \tilde{x}=f(t, \tilde{x})$.

Para o caso em que a derivada é um operador de Caputo, a definição é análoga à Definição 3.11

Definição 3.12 A constante $\tilde{x}$ é um ponto de equilíbrio do sistema

$$
\left\{\begin{array}{c}
{ }_{\mathrm{t}_{0}}^{\mathrm{C}} \mathrm{D}_{t}^{\alpha} x(t)=f(t, x(t)) \\
x\left(t_{0}\right)=x_{t_{0}}
\end{array}\right.
$$

se e somente se $f(t, \tilde{x})=0$, onde $\alpha \in(0,1), x=\left(x_{1}, x_{2}, \ldots, x_{n}\right)^{T} \in \Re^{n}, t_{0} \geqslant 0$ o tempo inicial e $f:[0,+\infty] \times \Re^{n} \rightarrow \Re^{n}$ contínua por partes em $t$ satisfazendo a condição de Lipschitz local em $x$.

Uma observação importante é que o conceito de ponto de equilíbrio só faz sentido quando estes pontos representam as soluções estacionárias, isto é, $x(t)=\tilde{x}$ resolve o sistema de EDOF. Assim, 
a derivada de $x\left(t_{0}\right)$ é igual a zero. Para a derivada de Caputo isso é sempre verdade, mas vimos que a derivada de Riemann-Liouville de uma constante pode ser uma constante diferente de zero. Deste modo, o conceito de ponto de equilíbrio para sistemas com operador de Riemann-Liouville apenas fará sentido quando esta derivada para uma constante for zero.

Podemos supor, sem perda de generalidade, que o ponto de equilíbrio do Sistema 3.42 é a origem. A razão disto é que qualquer ponto de equilíbrio pode ser transladado para a origem via mudança de variáveis. Se o ponto de equilíbrio é $\tilde{x} \neq 0$, com a mudança de variável $y(t)=x(t)-\tilde{x}$, então os Sistemas 3.11 e 3.42 podem ser reescritos como

$$
\begin{aligned}
{ }_{\mathrm{t}_{0}} \mathrm{D}_{t}^{\alpha} y(t) & ={ }_{\mathrm{t}_{0}} \mathrm{D}_{t}^{\alpha}(x(t)-\tilde{x})=f(t, x(t))-\frac{\bar{x} t^{-\alpha}}{\Gamma(1-\alpha)} \\
& =f(t, y+\bar{x})-\frac{\bar{x} t^{-\alpha}}{\Gamma(1-\alpha)}=g(t, y)
\end{aligned}
$$

onde ${ }_{t_{0}} \mathrm{D}_{t}$ representa tanto a derivada de Riemann-Liouville quanto a de Caputo e $g(t, 0)=0$ e o novo sistema tem ponto de equilíbrio na origem para a variável $y$.

\subsubsection{Estabilidade de Mittag-Leffler}

A definição de estabilidade para pontos de equilíbrio em sistemas de ordem fracionária é análoga aos sistemas de ordem inteira. Os Sistemas 3.41 e 3.42 são estáveis se, dada as condições iniciais $x(0) \in \mathbb{R}^{n}$, existe $\lambda>0$ tal que a solução $x(t)$ de tais sistemas satisfaz $\|x(t)\|\left\langle\lambda\right.$ para todo $t>t_{0}$ . Além disso, é assintoticamente estável se $\|x(t)\| \rightarrow 0$ quando $t \rightarrow \infty$.

Devido ao fato dos sistemas autônomos de ordem fracionária terem recursos de memória, a taxa de decaimento de tais sistemas é anômala, diferente de sistemas de ordem inteira. Assim não podemos aplicar os métodos clássico de análise qualitativa para caracterizar o fluxo de EDO's fracionárias. Por exemplo, a estabilidade exponencial não pode ser usada para caracterizar a estabilidade assintótica de sistemas fracionários [2]. Vejamos inicialmente um exemplo que pode caracterizar bem o comportamento de sistemas com ordem fracionária.

Exemplo 3.4 Considere os dois sistemas seguintes com condição inicial $x(0)$ para $0<\alpha<1$.

$$
\begin{gathered}
\frac{d}{d t} x(t)=a t^{a-1}, \\
{ }_{0}^{\mathrm{C}} \mathrm{D}_{t}^{\alpha} x(t)=a t^{a-1}, 0<\alpha<1 .
\end{gathered}
$$

Podemos ver que as soluções analíticas dos dois sistemas são

$$
t^{a}+x(0) \quad \text { e } \quad \frac{a \Gamma(a) t^{a+\alpha-1}}{\Gamma(a+\alpha)}+\frac{x(0) t^{\alpha-1}}{\Gamma(\alpha)}
$$

respectivamente. O Sistema 3.43 é instável para qualquer $a \in(0,1)$, pois no ponto de equilíbrio do sistema $(t=0), \lim _{t \rightarrow \infty}\left(t^{a}+x(0)\right)=\infty$ mas o Sistema 3.44 é estável no caso em que $\lim _{t \rightarrow \infty} t^{a+\alpha-1}$ converge para zero, ou seja, para $0<a<1-\alpha$. Isso mostra que, de fato, sistemas de ordem fracionária podem ter características de comportamento além dos sistemas de ordem inteira.

Definiremos agora alguns resultados que são pré requisitos para o estudo da estabilidade de Mittag-Leffler.

Lema 3.8 [32] A integral fracionária da função $f(t, x)$ dos Sistemas 3.42 ou 3.41 satisfaz a seguinte desigualdade:

$$
\left\|t_{t_{0}} J_{t}^{\alpha} f(t, x(t))\right\| \leq{ }_{t_{0}} J_{t}^{\alpha}\|f(t, x(t))\|,
$$

onde $\alpha \geq 0$ e $\|\cdot\|$ denota uma norma arbitrária. 
Demonstração: Com uma norma arbitrária para integrais de ordem fracionária, podemos então escrever

$$
\begin{gathered}
\left\|_{t_{0}} J_{t}^{\alpha} f(t, x(t))\right\|=\left\|\frac{1}{\Gamma(\alpha)} \int_{t_{0}}^{t} \frac{f(\tau, x(\tau))}{(t-\tau)^{1-\alpha}} d \tau\right\| \\
\leq \frac{1}{\Gamma(\alpha)} \int_{t_{0}}^{t} \frac{\|f(\tau, x(\tau))\|}{(t-\tau)^{1-\alpha}} d \tau \\
={ }_{t_{0}} J_{t}^{\alpha}\|f(t, x(t))\|
\end{gathered}
$$

o que termina a prova.

Lema 3.9 (Princípio da Comparação para a derivada de Caputo) $S e_{\mathrm{t}_{0}}^{\mathrm{C}_{t}} \mathrm{D}_{t}^{\alpha} x(t) \geq{ }_{\mathrm{t}_{0}}^{\mathrm{C}} \mathrm{D}_{t}^{\alpha} y(t)$ $e 0<\alpha<1, x(0)=y(0)$, então $x(t) \geq y(t)$.

Demonstração. Suponha que ${ }_{t_{0}}^{C} D_{t}^{\alpha} x(t)=f(t, x(t))$ e ${ }_{t_{0}}^{C} D_{t}^{\alpha} y(t)=g(t, y(t))$. Então, por hipótese temos que $f(t, x(t)) \geq g(t, y(t))$. Da equação integral de Volterra equivalente (3.29), temos que

$$
\begin{aligned}
& y(t)=y(0)+\frac{1}{\Gamma(\alpha)} \int_{t_{0}}^{t}(t-\tau)^{\alpha-1} g(\tau, y(\tau)) d \tau \\
& x(t)=x(0)+\frac{1}{\Gamma(\alpha)} \int_{t_{0}}^{t}(t-\tau)^{\alpha-1} f(\tau, x(\tau)) d \tau .
\end{aligned}
$$

Como $x(0)=y(0)$, substituindo $y(0)$ de (3.47) em (3.46), obtemos

$$
y(t)=x(t)-\frac{1}{\Gamma(\alpha)} \int_{t_{0}}^{t}(t-\tau)^{\alpha-1}(g(\tau, x(\tau))-f(\tau, x(\tau))) d \tau .
$$

Desde que $0<\tau<t \Rightarrow t-\tau>0,0<\alpha<1 \Rightarrow \Gamma(\alpha)>0$ e $g(t, x(t)-f(t, x(t) \leq 0$, concluímos de (3.48) que $y(t) \leq x(t)$.

Teorema 3.10 [32, 33] Se $x=0$, o ponto de equilíbrio do sistema,

$$
{ }_{\mathrm{t}_{0}}^{\mathrm{C}} D_{t}^{\alpha} x(t)=f(t, x),
$$

onde $f$ é Lipschitz em relação a $x$ por uma constante $L$ e contínua por partes com relação a $t$, então a solução do sistema satisfaz $\|x(t)\| \leq\left\|x\left(t_{0}\right)\right\| E_{\alpha}\left(L\left(t-t_{0}\right)^{\alpha}\right)$, onde $\alpha \in(0,1)$.

Demonstração: Primeiramente, aplicamos $t_{0} J_{t}^{\alpha} f(t, x(t))$ em ambos os lados da equação (3.49), onde podemos escrever

$$
\begin{gathered}
t_{0} J_{t t_{0}}^{\alpha} J_{t}^{0-\alpha} D^{0} x(t)={ }_{t_{0}} J_{t}^{\alpha} f(t, x(t)) \\
x(t)=x\left(t_{0}\right)+{ }_{t_{0}} J_{t}^{\alpha} f(t, x(t)) .
\end{gathered}
$$

Usando a desigualdade da norma, podemos escrever a expressão acima como,

$$
\|x(t)\|-\| x\left(t_{0}\|\leq\| x(t)-x\left(t_{0}\right)\|\leq\| t_{t_{0}} J_{t}^{\alpha} f(t, x(t)) \|,\right.
$$

Agora, usando a condição de Lipschitz e o Lema 3.8, temos,

$$
\left\|t_{t_{0}} J_{t}^{\alpha} f(t, x(t))\right\| \leq t_{0} J_{t}^{\alpha}\|f(t, x(t))\| \leq L_{t_{0}} J_{t}^{\alpha}\|x(t)\| .
$$

Nesse caso, existe uma função não negativa $m(t)$ satisfazendo,

$$
\left\|x(t)-x\left(t_{0}\right)\right\|=L_{t_{0}} J_{t}^{\alpha}\|x(t)\|-m(t)
$$


Aplicando a transformada de Laplace na equação acima, temos

$$
\|X(s)\|=\frac{\| x\left(t_{0} \| s^{\alpha-1}-s^{\alpha} M(s)\right.}{s^{\alpha}-L}
$$

onde $\|X(s)\|=\mathcal{L}(\|x(t)\|)$. Aplicando a transformada de Laplace inversa em (5.12), temos

$$
\|x(t)\|=\left\|x\left(t_{0}\right)\right\| E_{\alpha}\left(L\left(t-t_{0}\right)^{\alpha}\right)-m(t) *\left[\left(t-t_{0}\right)^{-1} E_{\alpha, 0}\left(L\left(t-t_{0}\right)^{\alpha}\right)\right],
$$

sendo * o operador de convolução. Devido ao fato de

$$
\begin{aligned}
\frac{d E_{\alpha}\left(L\left(t-t_{0}\right)^{\alpha}\right)}{d t} & =\frac{d}{d t}\left(\frac{1}{\Gamma(1)}+\frac{L\left(t-t_{0}\right)^{\alpha}}{\Gamma(\alpha+1)}+\frac{L^{2}\left(t-t_{0}\right)^{2 \alpha}}{\Gamma(2 \alpha+1)}+\ldots\right) \\
& =0+\frac{\alpha L\left(t-t_{0}\right)^{\alpha-1}}{\alpha \Gamma(\alpha)}+\frac{2 \alpha L^{2}\left(t-t_{0}\right)^{2 \alpha-1}}{2 \alpha \Gamma(2 \alpha)}+\ldots \\
& =\left(t-t_{0}\right)^{-1}\left(\frac{1}{\Gamma(0)}+\frac{L\left(t-t_{0}\right)^{\alpha}}{\Gamma(\alpha)}+\frac{L^{2}\left(t-t_{0}\right)^{2 \alpha}}{\Gamma(2 \alpha)}+\ldots\right) \\
& =\left(t-t_{0}\right)^{-1}\left(\sum_{k=0}^{\infty} \frac{\left(L\left(t-t_{0}\right)^{\alpha}\right)^{k}}{\Gamma(\alpha k)}\right)=\left(t-t_{0}\right)^{-1} E_{\alpha, 0}\left(L\left(t-t_{0}\right)^{\alpha}\right)
\end{aligned}
$$

A partir da igualdade acima e devido ao fato de $0 \leq \frac{d E_{\alpha}\left(L\left(t-t_{0}\right)^{\alpha}\right)}{d t}$ (lembrando que $L$ é uma constante de Lipschitz, e portanto maior que 0), temos

$$
\left(t-t_{0}\right)^{-1} E_{\alpha, 0}\left(L\left(t-t_{0}\right)^{\alpha}\right)=\frac{d E_{\alpha}\left(L\left(t-t_{0}\right)^{\alpha}\right)}{d t} \geqslant 0,
$$

que leva a

$$
\|x(t)\| \leq\left\|x\left(t_{0}\right)\right\| E_{\alpha}\left(L\left(t-t_{0}\right)^{\alpha}\right) .
$$

No caso particular em que $\alpha=1$, obtemos a desigualdade

$$
\|x(t)\| \leq\left\|x\left(t_{0}\right)\right\| e^{L\left(t-t_{0}\right)},
$$

que é equivalente à desigualdade (3.36).

Como aponta Chen (2009) [59], o decaimento da energia generalizada de um sistema dinâmico não precisa ser exponencial para que o sistema seja estável. O decaimento de energia pode ser de qualquer taxa, incluindo decaimento em lei de potência. Para estender a aplicação do cálculo fracionário em sistemas não lineares, Li et al. (2009) [32] propõe a estabilidade de Mittag Leffler, cujo objetivo é caracterizar a velocidade de decaimento da função de Lyapunov de uma maneira mais geral que inclua a estabilidade exponencial e a estabilidade em lei de potência como casos especiais.

Definição 3.13 (Estabilidade de Mittag-Leffler) A solução do Sistema 3.42 é dita ser MittagLeffler estável se

$$
\|x(t)\| \leq\left(m\left(x\left(t_{0}\right)\right) E_{\alpha}\left(-\lambda\left(t-t_{0}\right)^{\alpha}\right)^{b}\right.
$$

onde $t_{0}$ é o tempo inicial, $\alpha \in(0,1), \lambda>0, b>0, m(0)=0, m(x)>0$ e $m(x)$ localmente Lipschitziana em $x \in B \in \Re^{n}$ com constante de Lipschitz $m_{0}$.

Duas observações importantes devem ser feitas. A primeira é que a estabilidade de MittagLeffler implica estabilidade assintótica. Isso acontece por que no lado direito da desigualdade 3.51. $m\left(x\left(t_{0}\right)\right)$ é finito e para $\alpha<1, E_{\alpha}\left(-\lambda\left(t-t_{0}\right)^{\alpha}\right) \rightarrow 0$ quando $t \rightarrow \infty$. Não somente isso, pois a estabilidade de Mittag-Leffler mostra uma convergência mais rápida que a estabilidade exponencial 
próxima à origem, como pode ser visto a partir das duas derivadas

$$
\left\{\frac{d}{d t} e^{-\lambda t}\right\}_{t=0}=-\left.\lambda e^{-\lambda t}\right|_{t=0}=-\lambda \quad \text { e } \quad\left\{\frac{d}{d t} E_{\alpha}\left(-\lambda t^{\alpha}\right)\right\}_{t=0}=-\infty
$$

onde $\alpha \in(0,1)$ e $\lambda>0$. Devido ao fato de $e^{0}=E_{\alpha}(0)=1$, segue do princípio da comparação que $E_{\alpha}\left(-\lambda t^{\alpha}\right)$ decresce muito mais rapidamente que $e^{-\lambda t}$ próximo à origem. A segunda observação é que a expressão no lado direito de (3.51) é completamente monotônica para $0<\alpha<1$, e portanto maior ou igual a zero.

Há ainda uma generalização da Definição 3.13, que usa a função de Mittag-Leffler de dois parâmetros.

Definição 3.14 (Estabilidade de Mittag-Leffer Generalizada) [33] A solução do Sistema 3.42 é Mittag-Leffler estável generalizado se

$$
\|x(t)\| \leq\left[m\left(x_{t_{0}}\right)\left(t-t_{0}\right)^{-\gamma} E_{\alpha, 1-\gamma}\left(-\lambda\left(t-t_{0}\right)^{\alpha}\right]^{b}\right.
$$

Na desigualdade 3.52 , para $\lambda=0$, temos

$$
\|x(t)\| \leq\left[\frac{m\left(x_{t_{0}}\right)}{\Gamma(1-\gamma)}\right]^{b}\left(t-t_{0}\right)^{-\gamma b}
$$

o que mostra que a estabilidade polinomial é um caso especial da estabilidade de Mittag-Leffler.

\subsubsection{Método direto de Lyapunov para sistemas fracionários}

Segundo Li, Chen (2010)[33], para sistemas não lineares de equações de ordem fracionária, o método direto de Lyapunov fornece-nos uma maneira de analisar a estabilidade desse tipo de sistemas sem explicitar as soluções, desde que, exista alguma candidata à função de Lyapunov. Vamos agora apresentar um teorema que nos permite encontrar uma função de Lyapunov tal que o sistema estudado tenha um ponto de equilíbrio que seja Mittag-Leffler estável.

Teorema 3.11 (Método Direto de Lyapunov para Sistemas Fracionários) [33] Seja $x=$ 0 o ponto de equilíbrio do Sistema 3.42 e $D \in \Re^{n}$ um domínio contendo a origem. Seja $V(t, x(t))$ : $[0, \infty] \times D \rightarrow \Re$ uma função continuamente diferenciável e localmente Lipschitziana com respeito a $x$ tal que

$$
\begin{gathered}
\alpha_{1}\|x\|^{a} \leq V(t, x) \leq \alpha_{2}\|x\|^{a b}, \\
{ }_{0}^{C} D_{t}^{\alpha} V(t, x(t)) \leq-\alpha_{3}\|x\|^{a b}
\end{gathered}
$$

onde $t \geq 0, \alpha \in(0,1), x \in B, \alpha_{1}, \alpha_{2}, \alpha_{3}, a$ e b são contantes arbitrárias positivas. Então o ponto de equilíbrio $\bar{x}=0$ é globalmente Mittag-Leffler estável.

Demonstração. Segue direto das Equações (3.53) e (3.54) que

$$
{ }_{0}^{\mathrm{C}} \mathrm{D}_{t}^{\alpha} V(t, x(t)) \leq-\frac{\alpha_{3}}{\alpha_{2}} V(t, x(t))
$$

A partir de (3.55), vemos que há uma função não negativa $m(t)$ tal que

$$
{ }_{0}^{\mathrm{C}} \mathrm{D}_{t}^{\alpha} V(t, x(t))+m(t)=-\alpha_{3} \alpha_{2}^{-1} V(t, x(t))
$$

Tomando a transformada de Laplace de 3.56 , temos

$$
\begin{gathered}
s^{\alpha} V_{L}(s)-V(0) s^{\alpha-1}+M(s)=-\alpha_{3} \alpha_{2}^{-1} V_{L}(s), \\
V_{L}(s)=\frac{V(0) s^{\beta-1}-M(s)}{s^{\beta}+\frac{\alpha_{3}}{\alpha_{2}}}
\end{gathered}
$$


onde $V(0)=V(0, x(0))$ é uma constante não negativa e $V_{L}(s)=\mathcal{L}(V(t, x(t))), M(s)=\mathcal{L}(M(t))$. Caso $x(0)=0$, então $V(0)=0$, o que leva à solução $x(t)=0$ para a Equação $(3.42)$. Se $x(0) \neq 0$, então a função $V(0)$ é não negativa, ou seja, $V(0)>0$. Levando em conta que $V(t, x)$ é localmente Lispschitziana com respeito a $x$ e do teorema de existência e unicidade, aplicando a transformada de Laplace inversa, obtemos a única solução da Equação (3.57)

$$
V(t)=V(0) E_{\alpha}\left(\frac{-\alpha_{3}}{\alpha_{2}} t^{\alpha}\right)-m(t) *\left[t^{\alpha-1} E_{\alpha, \alpha}\left(\frac{-\alpha_{3}}{\alpha_{2}} t^{\alpha}\right)\right]
$$

Note que

$$
\begin{aligned}
& -\alpha \frac{d}{d x} E_{\alpha}\left(-\alpha_{3} \alpha_{2}^{-1} t^{\alpha}\right)=-\alpha \frac{d}{d x} \sum_{k=0}^{\infty} \frac{\left(-\alpha_{3} \alpha_{2}^{-1} t^{\alpha}\right)^{k}}{\Gamma(\alpha k+1)} \\
& -\alpha\left(\frac{-1}{\Gamma(\alpha+1)}+\frac{2\left(-\alpha_{3} \alpha_{2}^{-1} t^{\alpha}\right)}{\Gamma(2 \alpha+1)}+\frac{3\left(-\alpha_{3} \alpha_{2}^{-1} t^{\alpha}\right)^{2}}{\Gamma(3 \alpha+1)}+\ldots\right) \\
& -\alpha\left(\frac{-1}{\alpha \Gamma(\alpha)}+\frac{2\left(-\alpha_{3} \alpha_{2}^{-1} t^{\alpha}\right)}{2 \alpha \Gamma(2 \alpha)}+\frac{3\left(-\alpha_{3} \alpha_{2}^{-1} t^{\alpha}\right)^{2}}{3 \alpha \Gamma(3 \alpha)}+\ldots\right) \\
& =\frac{-1}{\Gamma(\alpha)}+\frac{\left(-\alpha_{3} \alpha_{2}^{-1} t^{\alpha}\right)}{\Gamma(\alpha+\alpha)}+\frac{\left(-\alpha_{3} \alpha_{2}^{-1} t^{\alpha}\right)^{2}}{\Gamma(2 \alpha+\alpha)}+\ldots \\
& =E_{\alpha, \alpha}\left(-\frac{\alpha_{3}}{\alpha_{2}} t^{\alpha}\right)
\end{aligned}
$$

e como $\frac{d}{d x} E_{\alpha}\left(-\alpha_{3} \alpha_{2}^{-1} t^{\alpha}\right) \leq 0$ (do Teorema 3.1. esta função é completamente monotônica), implica que $E_{\alpha, \alpha}\left(-\frac{\alpha_{3}}{\alpha_{2}} t^{\alpha}\right) \geq 0$. Além disso $t^{\alpha-1}>0$, o que leva a

$$
V(t) \leq V(0) E_{\alpha}\left(-\alpha_{3} \alpha_{2}^{-1} t^{\alpha}\right) .
$$

Substituindo essa equação em 3.53 segue que

$$
\|x(t)\| \leq\left[\frac{V(0)}{\alpha_{1}} E_{\alpha}\left(-\frac{\alpha_{3}}{\alpha_{2}} t^{\alpha}\right)\right]^{\frac{1}{a}},
$$

onde $\frac{V(0)}{\alpha_{1}}>0$ para $x(0) \neq 0$.Se $m=\frac{V(0)}{\alpha_{1}}=\frac{V(0, x(0)}{\alpha_{1}} \geq 0$, então temos

$$
\|x(t)\| \leq\left[m E_{\beta}\left(-\frac{\alpha_{3}}{\alpha_{2}} t^{\beta}\right)\right]^{\frac{1}{a}}
$$

onde $m=0$ é assegurado se e somente $x(0)=0$. Como $V(t, x(t))$ é localmente Lipschitziana com respeito à $x$ e $V(0, x(0))=0$ se e somente se $x(0)=0$, segue que $m=\frac{V(0, x(0))}{\alpha_{1}}$ é também localmente Lipschitz com respeito à $x$ e $m(0)=0$, o que leva à estabilidade de Mittag-Leffler para o Sistema 3.42 .

Para o Sistema (3.41), onde o operador fracionário é de Riemann-Liouville, podemos usar a composição dos operadores de Riemann-Liouville e Caputo (Equação (2.22)

$$
{ }_{0}^{\mathrm{C}} \mathrm{D}_{t}^{\alpha} m(t)={ }_{0}^{R L} D_{t}^{\alpha-1} m^{\prime}(t)={ }_{0}^{\mathrm{RL}} \mathrm{D}_{t}^{\alpha} m(t)-\frac{m(0) t^{-\alpha}}{\Gamma(1-\alpha)} .
$$

Devido ao fato de $\alpha \in(0,1)$ e $m(0) \geq 0$, segue de (3.61) que

$$
{ }_{0}^{\mathrm{C}} \mathrm{D}_{t}^{\alpha} m(t) \leq{ }_{0}^{\mathrm{RL}} \mathrm{D}_{t}^{\alpha} m(t) .
$$


Agora, como $V(t, x(t)) \geq 0$, temos que

$$
{ }_{0}^{\mathrm{C}} \mathrm{D}_{t}^{\alpha} V(t, x(t)) \leq{ }_{0}^{\mathrm{RL}} \mathrm{D}_{t}^{\alpha} V(t, x(t)),
$$

e desta maneira se substituirmos o operador ${ }_{0}^{C} \mathrm{D}_{t}^{\alpha}$ por ${ }_{0}^{\mathrm{RL}} \mathrm{D}_{t}^{\alpha}$ em 3.54 temos,

$$
{ }_{0}^{\mathrm{C}} \mathrm{D}_{t}^{\alpha} V(t, x(t)) \leq{ }_{0}^{\mathrm{RL}} \mathrm{D}_{t}^{\alpha} V(t, x(t)) \leq-\alpha_{3}\|x\|^{a b},
$$

e assim, procede-se de forma análoga à demonstração do Sistema 3.42.

Exemplo 3.5 [33] Considere o seguinte sistema

$$
{ }_{0}^{\mathrm{RL}} \mathrm{D}_{t}^{\alpha}|x(t)|=-|x(t)|
$$

onde $\alpha \in(0,1)$. Escolhemos como função de Lyapunov $V(t, x)=|x|$. Claramente essa função é Lipschitziana. Da Equação (3.62), temos que

$$
{ }_{0}^{\mathrm{C}} \mathrm{D}_{t}^{\alpha} V={ }_{0}^{\mathrm{C}} \mathrm{D}_{t}^{\alpha}|x(t)| \leq{ }_{0}^{\mathrm{RL}} \mathrm{D}_{t}^{\alpha}|x(t)|=-|x| .
$$

Supondo $\alpha_{1}=1, \alpha_{2}=1$ e $\alpha_{3}=-1$, satisfazemos as condições do Teorema 3.11, o que leva a

$$
|x(t)| \leq|x(0)| E_{\alpha}\left(-t^{\alpha}\right) .
$$

Utilizando a transformada de Laplace em (5.10), temos

$$
s^{\alpha} \mathcal{L}(|x(t)|)-\left[{ }_{0}^{R L} D_{t}^{\alpha-1}|x(t)|\right]_{t=0}=-\mathcal{L}(|x(t)|) .
$$

Aplicando a transformada de Laplace inversa, vamos obter

$$
|x(t)|=\left[{ }_{0} J_{t}^{1-\alpha}|x(t)|\right]_{t=0} E_{\alpha}\left(-t^{\alpha}\right),
$$

onde, da integral de Riemann-Liouville, temos que $\left[{ }_{0} J_{t}^{1-\alpha}|x(t)|\right]_{t=0}=0$, para qualquer $x(0)$ finito. Isso mostra que a estabilidade do Sistema 3.63 não pode ser encontrada diretamente calculando a sua solução.

Há ainda um outro teorema que nos dá condições para estabilidade assintótica de um ponto de equilíbrio de uma EDOF usando funções de classe K.

Definição 3.15 (Função Classe-K) [28] Uma função contínua $\alpha:[0, t) \rightarrow[0, \infty)$ é dita pertencer à classe-K se $\alpha(x)>0$ para todo $x>0$, é estritamente crescente e $\alpha(0)=0$. Além disso, se $t=\infty$ e $\alpha(x) \rightarrow \infty$ quando $x \rightarrow \infty$, então $\alpha$ é uma função classe $K_{\infty}$.

Lema 3.10 Sejam $\alpha_{1}$ e $\alpha_{2}$ funções de classe $K$ em $[0, \infty)$ e $\alpha_{3}$ e $\alpha_{4}$ funções de classe $K_{\infty}$. Seja também $\alpha_{i}^{-1}$ a inversa de $\alpha_{i}, i=1, \ldots, 4$. Então, 1) $\alpha_{1}^{-1}$ é definida em $\left[0, \alpha_{1}(a)\right)$ e é de classe $K$; 2) $\alpha_{3}^{-1}$ é definida em $\left[0, \alpha_{1}(a)\right)$ e é de classe $K_{\infty}$; 3) $\alpha_{1} \circ \alpha_{2}$ é de classe $K$; 4) $\alpha_{3} \circ \alpha_{4}$ é de classe $K_{\infty}$.

Teorema 3.12 [32] Seja $x=0$ o ponto de equilíbrio do Sistema 3.42) e $V(t, x(t)):[0, \infty) \times D \rightarrow$ $\mathbb{R}$ uma função de Lyapunov, onde $D$ é o domínio contendo a origem. Além disso, sejam $\alpha_{1}, \alpha_{2} e$ $\alpha_{3}$ funçôes de classe $K$ satisfazendo

$$
\begin{gathered}
\alpha_{1}(\|x\|) \leq V(t, x(t)) \leq \alpha_{2}(\|x\|) \\
{ }_{0}^{C} D_{t}^{\alpha} V(t, x(t)) \leq-\alpha_{3}(\|x\|),
\end{gathered}
$$

onde $\alpha \in(0,1)$. Então o ponto de equilíbrio do sistema 3.42 é assintoticamente estável. 
Demonstração. Segue das Equações (3.64), (3.65) e do fato de $\alpha_{2}$ e $\alpha_{3}$ serem estritamente crescente que

$$
\begin{aligned}
& \|x\| \geq \alpha_{2}^{-1}(V) \\
& { }_{0}^{\mathrm{C}} \mathrm{D}_{t}^{\alpha} V \leq-\alpha_{3}\left(\alpha_{2}^{-1}(V)\right) .
\end{aligned}
$$

Do princípio da comparação (Lema 3.9. temos que $V(t, x(t))$ é limitado pela única solução da equação diferencial escalar

$$
{ }_{0}^{\mathrm{C}} \mathrm{D}_{t}^{\alpha} g(t)=-\alpha_{3}\left(\alpha_{2}^{-1}(g(t))\right), g(0)=V(0, x(0)),
$$

e como, pelo Lema 3.10 $\alpha_{3} \alpha_{2}^{-1}$ é uma função classe $K$, temos que $g(t)=0$ para $t \geq 0$ se $g(0)=$ $V(0, x(0))=0$ e da definição de ponto de equilíbrio, $g=0$ é um ponto de equilíbrio do Sistema 3.66. Caso $g(t) \geq 0, t \in[0, \infty)$, segue de (3.66) que ${ }_{0}^{C} \mathrm{D}_{t}^{\alpha} g(t) \leq 0$. Assim, pelo princípio da comparação novamente,

$$
g(t) \leq g(0)
$$

para $t \in(0, \infty)$. Suponha por contradição que $g=0$ não é assintoticamente estável. Então existe uma constante positiva $\epsilon$ tal que

$$
0<\epsilon<g(t) \leq g(0), t \leq 0
$$

e ao substituir (3.67) em (3.66), nos leva a concluir que

$$
\begin{aligned}
-\alpha_{3}\left(\alpha_{2}^{-1}(g(t))\right) & \leq-\alpha_{3}\left(\alpha_{2}^{-1}(\epsilon)\right) \\
& =-\frac{\alpha_{3}\left(\alpha_{2}^{-1}(\epsilon)\right)}{g(0)} g(0) \\
& \leq-\lg (0),
\end{aligned}
$$

$\operatorname{com} 0<l=\frac{\alpha_{3}\left(\alpha_{2}^{-1}(\epsilon)\right)}{g(0)}$. Assim,

$$
{ }_{0}^{\mathrm{C}} \mathrm{D}_{t}^{\alpha} g(t)=-\alpha_{3}\left(\alpha_{2}^{-1}(g(t))\right) \leq-l g(t),
$$

e seguindo o mesmo raciocínio feito para a desigualdade 3.55 , temos que

$$
g(t) \leq g(0) E_{\alpha}(-l t \alpha) \rightarrow 0 \text { quando } t \rightarrow 0
$$

e isso contradiz o fato de que $g(t)>\epsilon$. Como $V(t, x(t)$ é limitado por $g(t)$, segue de (3.64) que

$$
\|x(t)\| \leq \alpha_{1}^{-1}(V(t, x(t))) \leq \alpha_{1}^{-1}(g(t)),
$$

e como $\alpha_{1}^{-1}$ é de classe $K$ e de $3.69, \lim _{t \rightarrow \infty}\|x\|=0$.

Exemplo 3.6 Considere o seguinte sistema de ordem fracionária

$$
\begin{aligned}
& { }_{0}^{\mathrm{C}} \mathrm{D}_{t}^{\alpha} x_{1}=-x_{1}+\sin \left(x_{3}\right) x_{1} \\
& { }_{0}^{\mathrm{C}} \mathrm{D}_{t}^{\alpha} x_{2}=-x_{2}+e^{-t} \cos \left(x_{1}\right) x_{2} \\
& { }_{0}^{\mathrm{C}} \mathrm{D}_{t}^{\alpha} x_{3}=-x_{3},
\end{aligned}
$$

onde $0<\alpha<1$ e $x(t)=\left(x_{1}, x_{2}, x_{3}\right) \in \Re^{3}$.

Considere a função $V(t, x)=\frac{x_{1}^{2}+x_{2}^{2}+x_{3}^{2}}{2}$. Usando um resultado estudado em [14], no qual afirma que, quando $0<\alpha<1$ e $P \in \Re^{n \times n}$ uma matriz constante, positiva definida e simétrica, a seguinte 
desigualdade é assegurada:

$$
\frac{1}{2}{ }^{{ }^{\mathrm{C}}} \mathrm{D}_{t}^{\alpha}\left(x^{T}(t) P x(t)\right) \leq x^{T}(t) P_{\mathrm{t}_{0}}^{\mathrm{C}} \mathrm{D}_{t}^{\alpha} x(t), \quad t \geq t_{0} .
$$

Da equação 3.71, temos que

$$
\begin{aligned}
& { }_{0}^{\mathrm{C}} \mathrm{D}_{t}^{\alpha} V(t, x(t)) \leq x_{1}{ }_{0}^{\mathrm{C}} \mathrm{D}_{t}^{\alpha} x_{1}+x_{2}{ }_{0}^{\mathrm{C}} \mathrm{D}_{t}^{\alpha}+x_{3}{ }_{0}^{\mathrm{C}} \mathrm{D}_{t}^{\alpha} \\
& \quad=-x_{1}^{2}(t)+\sin \left(x_{3}(t)\right) x_{2}^{2}(t)-x_{2}^{2}(t)+x_{2}^{2}(t) e^{-t} \cos \left(x_{t}(t)\right)-x_{3}^{2} \\
& \quad \leq 0 .
\end{aligned}
$$

Como $\frac{1}{4}\|x\|_{2}^{2} \leq V(t, x(t)) \leq\|x\|_{2}^{2}$ e ${ }_{0}^{\mathrm{C}} \mathrm{D}_{t}^{\alpha} V(t, x(t)) \leq 0$, pelo Teorema 3.12 , o ponto de equilíbrio $(0,0,0)^{T}$ desse sistema é assintoticamente estável. 


\section{Capítulo 4}

\section{Redes Neurais Artificiais de Hopfield Fracionárias}

\subsection{Conceitos Básicos}

O primeiro passo para o surgimento das redes neurais artificiais veio em 1943, quando Warren McCulloch, um neurofisiologista, e um matemático, Walter Pitts, escreveram um artigo sobre como neurônios podem funcionar [41]. Segundo Haykin [20, podemos definir uma rede neural como um processador maciçamente distribuído paralelamente, constituído de unidades de processamento simples, que tem a propensão natural para armazenar conhecimento experimental e torná-lo disponível para o uso.

As redes neurais se assemelham ao cérebro por adquirir o conhecimento do ambiente a partir de um processo de aprendizagem e armazenamento utilizando forças sinápticas conhecidas como pesos sinápticos. Há basicamente dois tipos de redes neurais: estáticas (feedforward) e dinâmicas (recorrentes). As redes neurais estáticas podem ser interpretadas como operadores de transformação de representação, mas não são capazes de reutilizar a informação transformada, produzindo apenas mapeamentos estáticos. Devido a isso, as redes neurais estáticas possuem dificuldade em representar comportamento dinâmico. Por outro lado, nas redes neurais dinâmicas, o tempo é representado pelo seu efeito real no processamento. Quanto ao processo de aprendizado, existem diversos algoritmos, que ajustam os pesos sinápticos da rede a partir de um processo iterativo de treinamento.

A vantagem de uma rede neural recorrente é a maneira como ela armazena informação. Podemos citar como um exemplo a memória associativa, análoga à forma como um cérebro humano armazena informação. Uma memória associativa é um recurso importante para o comportamento inteligente. Baseia-se no fato de que a memória é endereçada através do seu conteúdo, ou seja, se um padrão é apresentado a uma memória associativa, ele retorna se esse padrão coincide com um padrão armazenado. A coincidência não precisa ser perfeita, no entanto. Uma memória associativa também pode retornar um padrão armazenado semelhante ao apresentado, de modo que a entrada ruidosa também possa ser reconhecida. [20]

O padrão não corrompido é usado como um ponto de equilíbrio estável e suas versões ruidosas como sua bacia de atração. Desta forma, uma rede neural dinâmica associada a um conjunto de padrões é criado. Se todo o espaço de trabalho é corretamente particionado por uma memória endereçável por conteúdo, então um sistema deve ter uma solução de estado estacionário correspondente ao padrão não corrompido para qualquer condição inicial que represente um padrão de amostra. A dinâmica da rede neural de tal classificador serve como um filtro para "limpar"os dados corrompidos. 19

As redes de Hopfield, apresentadas por J. Hopfield [42], são um tipo especial de redes neurais recorrentes que podem ser usadas como memória associativa. Segundo Hopfield, alguns modelos de sistemas físicos poderiam ser usados para resolver problemas computacionais. Tais sistemas podem ser implementados em hardware, combinando componentes comuns, como capacitores e resistores.

Para entendermos melhor o funcionamento das redes neurais de Hopfield, vamos começar ex- 
plicando o funcionamento de um neurônio biológico. Os neurônios são células especializadas na recepção, condução e transmissão de sinais eletroquímicos. Existem 100 bilhões de neurônios e cerca de 100 trilhões de sinapses entre eles [46]. Eles vêm em uma grande variedade de formas, tamanhos e propriedades, mas compartilham características anatômicas típicas, como visto na Figura 4.1

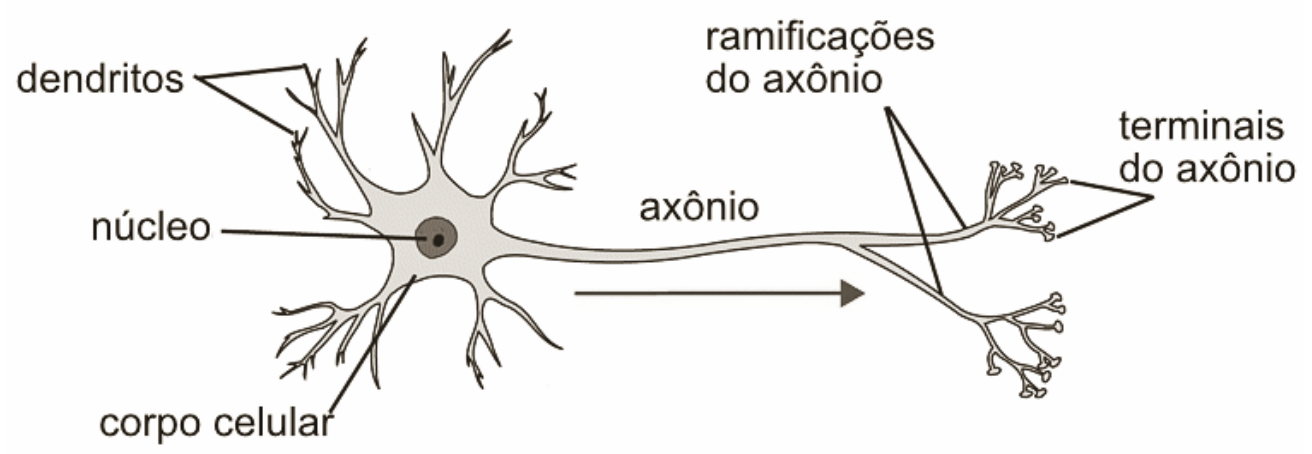

Figura 4.1: A anatomia de um neurônio.

O corpo celular constitui o centro metabólico e contém o núcleo da célula. Muitas extensões chamadas de dendritos se ramificam no corpo da célula e recebem entrada de sinais sinápticos de outros neurônios. Um único corpo cilíndrico longo chamado de axônio se projeta do corpo celular e ramifica-se em suas extremidades. A propagação do sinal eletroquímico nervoso é chamado de potencial de ação. Um potencial de ação é uma onda auto-regenerativa de atividade eletroquímica que permite que um neurônio possa transportar um sinal ao longo do comprimento do axônio para os ramos terminais, que formam conexões com neurônios adjacentes [26]. O potencial de ação ocorre somente se o estímulo elétrico ou químico tiver energia suficiente para superar o potencial limiar

A membrana do axônio é formada por duas camadas de moléculas lipídicas que separam o citoplasma intracelular do axônio e do fluido extracelular, dos canais iônicos (ou poros), enzimas, bombas e receptores. Os poros agem como portas na barreira lipídica, através dos quais as substâncias podem ser transferidas de um lado para o outro.

Em 1952, Hodgkin e Huxley [22], provaram a existência de um potencial elétrico através da membrana de um axônio. No estado de repouso, o citoplasma dentro de todos os neurônios contém uma composição iônica que torna o interior da célula mais negativo em potencial do que o exterior da célula. Tal voltagem é mantida a um gasto metabólico por bombas localizadas na membrana. As duas camadas de lípidos atuam como um isolador fino e assim a membrana do axônio tem a propriedade de capacitância, isto é, separação de carga. Portanto, o potencial de membrana (diferença entre as voltagens intracelulares e extracelulares) $V_{m}(t)=V_{\text {int }}(t)-V_{\text {ext }}(t)$, permite que a capacitância $C$ armazene uma carga $Q$. Da definição de capacitância, temos que

$$
Q=C V_{m}(t)
$$

onde C é uma grandeza escalar constante. Quando a tensão através do capacitor muda, uma corrente irá fluir. A corrente do capacitor $I_{C}$ é obtida pela diferenciação da Equação 4.1 em relação ao tempo, ou seja,

$$
I_{C}(t)=C \frac{d V_{m}(t)}{d t}
$$

Íons carregados (como $\mathrm{Ca}^{2+}$, por exemplo) passam pelas vesículas, que abrem ou fecham em resposta a condições locais como a voltagem através da membrana excitável (a resistência à penetração de íons varia conforme a diferença de potencial é alterada). A ligação dos neurotransmissores com os receptores de membrana é um processo muito rápido (de menos de $1 \mathrm{~ms}$ ) após o qual os neurotransmissores são liberados. A liberação é feita por células gliais especializadas, denominadas 
"transportadoras". As transportadoras removem os neurotransmissores da membrana pós sináptica e os transportam ao neurônio pré sináptico para que eles sejam rearmazenados nas suas vesículas. A Figura 4.2 ilustra esse processo de troca de informação entre o neurônio pós sináptico e pré sináptico. Hodgkin e Huxley [22] propuseram um modelo de circuito elétrico para um canal iônico

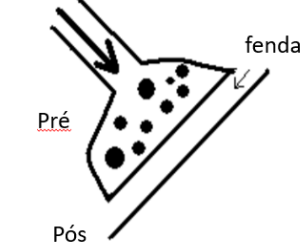

Um potencial de ação chega ao terminal sináptico

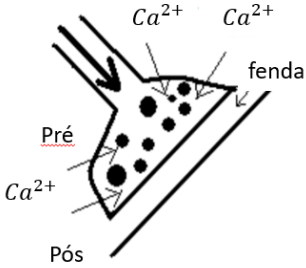

Íons de Cálcio entram na célula

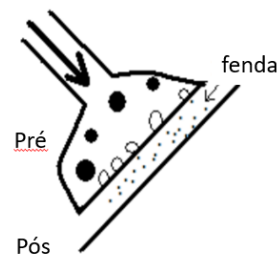

Algumas vesículas se fundem com a membrana pré-sináptica e liberam neurotransmissores, que se difundem pela fenda sináptica

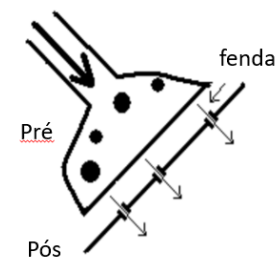

Os neurotransmissores provocam a abertura de canais iônicos que excitam ou inibem o neurônio póssináptico.

Figura 4.2: Ligação entre neurônios pós e pré sinápticos. Fonte: [51].

que consiste de uma bateria e um resistor em série. A bateria modela a força devido à diferença na concentração de íons dentro e fora da célula, e o resistor modela a permeabilidade do canal para o íon em específico. Então, pela lei de voltagem de Kirchhoff, a queda de voltagem através da membrana é igual à soma da queda de voltagem através da bateria $\left(E_{i o n}\right)$ e a queda de voltagem através do resistor:

$$
V_{m}=E_{i o n}+R I_{i o n},
$$

onde $I_{i o n}$ é a corrente através da resistência $R$, dada pela lei de Ohm. Portanto,

$$
I_{\text {ion }}(t)=\frac{V_{m}(t)-E_{i o n}(t)}{R} .
$$

A célula é então modelada por um circuito resistor-capacitor $(R C)$, como mostrado na Figura 4.3. Aplicando a Lei de Kirchhoff, nós temos $I_{C}+I_{i o n}=I_{e x t}$, e da Equação (4.3),

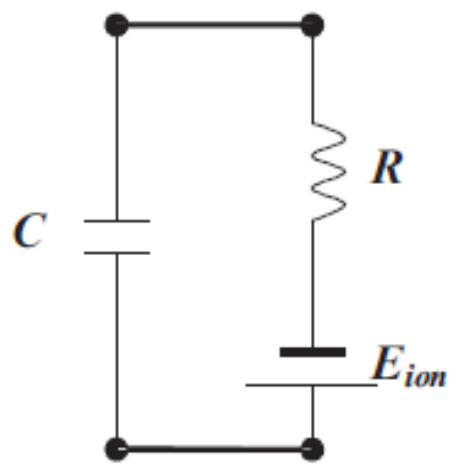

Figura 4.3: Modelagem de um neurônio por um circuito $R C$.

$$
\begin{gathered}
C \frac{d V_{m}(t)}{d t}=-I_{\text {ion }}(t)+I_{\text {ext }}(t) \\
C \frac{d V_{m}(t)}{d t}=-\frac{1}{R}\left(V_{m}(t)-E_{\text {ion }}(t)\right)+I_{\text {ext }}(t) .
\end{gathered}
$$

Para estender a equação acima para uma rede de $n$ neurônios, seja $I_{e x t_{k}}=I_{s i n_{k, j}}+I_{a p l_{k}}$ a corrente externa chegando do neurônio $k$, onde $I_{\sin _{k, j}}$ é a corrente sináptica que vem do neurônio $j$ para o neurônio $k$, e $I_{a p l_{k}}$ é a corrente aplicada experimentalmente ao neurônio $k$. Portanto, nós 
temos,

$$
C_{k} \frac{d V_{m_{k}}}{d t}=-I_{i o n_{k}}+\sum_{j=1, j \neq k}^{n} I_{s i n_{k, j}}+I_{a p l_{k}} .
$$

A corrente sináptica pode ser modelada por

$$
I_{\sin _{k, j}}=g_{\sin _{j}}\left(V_{m_{j}}\right)\left(V_{m_{k}}-V_{s i n}\right)
$$

onde $g_{s i n_{j}}$ representa a condutância sináptica (que descreve a variação na condutância dos canais sinápticos na membrana do neurônio pós sináptico) e depende da tensão pré sináptica $V_{m_{j}}, V_{s i n}$ representa o potencial de reversão (equilíbrio) sináptico e $V_{m_{k}}$ é a voltagem de membrana do neurônio pós-sináptico.

Há uma infinidade de modelos matemáticos que caracterizam os neurônios biológicos e tentam fornecer ideias sobre os processos que ocorrem dentro do neurônio ou para descrever as comunicações entre eles. Eles são construídos para ter nós correspondentes aos neurônios e conexões entre eles correspondentes às sinapses [26.

O modelo de um neurônio (nó) na rede é apresentado na Figura 4.4 20. Um sinal de entrada $x_{j}, j=1, \ldots, n$ na sinapse $j$ conectado ao neurônio $k$ é multiplicado pelo peso sináptico. Este peso sináptico representa a força da conexão e é positivo se for excitatório ou negativo se for inibitório. A matriz $\left[w_{k j}\right]$ formada pelas forças sinápticas é chamada matriz de conexão. A junção somadora é um combinador linear de todos os sinais de entrada.

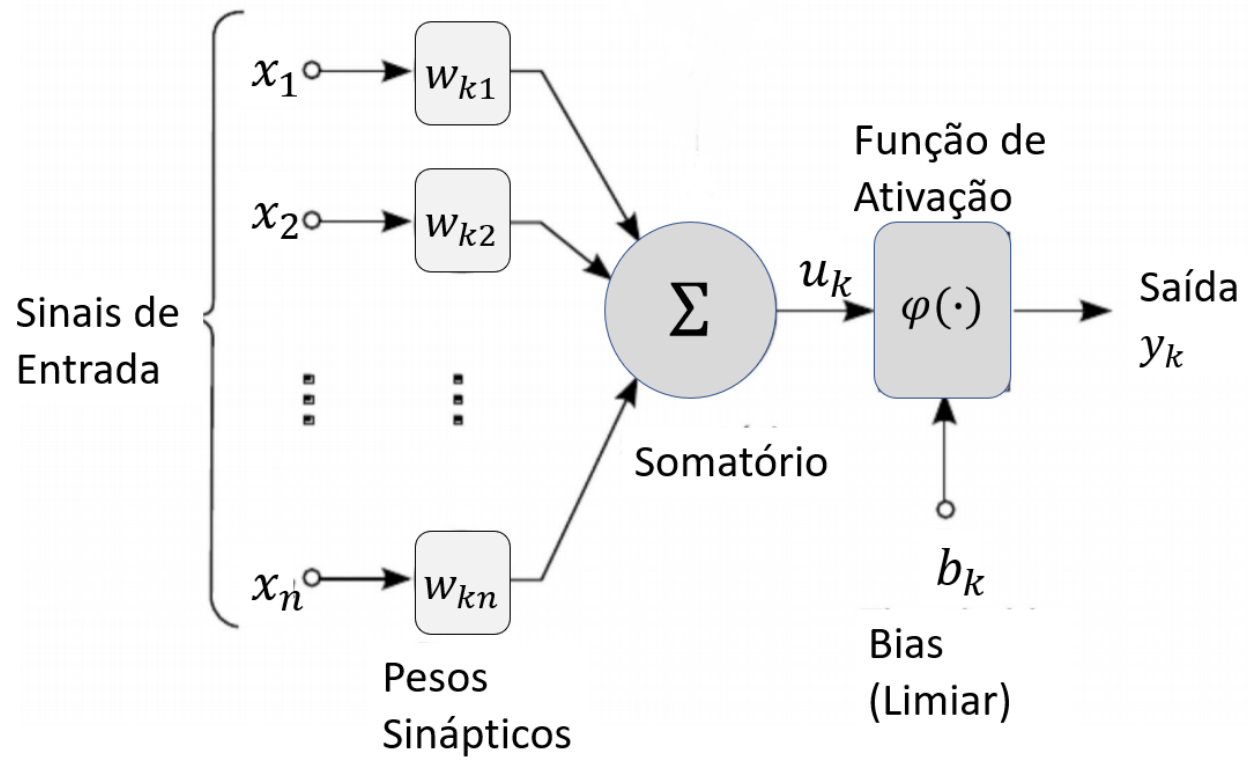

Figura 4.4: Modelo de um rede neural artificial.

A função de ativação $\varphi(\cdot)$ é um elemento não linear que possui a propriedade de limitar a amplitude do sinal de saída $y_{k}$

$$
\varphi\left(\sum_{j=1}^{n} w_{k j} x_{j}\right)=y_{k} .
$$

A função $\varphi(\cdot)$ tem por objetivo modelar a propriedade da não linearidade dos neurônios biológicos. É geralmente considerado monotonicamente crescente e diferenciável em $(-\infty, \infty)$, satisfazendo $\varphi(0)=0$ e $\varphi^{\prime}(z) \leq \varphi^{\prime}(0)$, para $z \in \mathbb{R}$, e

$$
\lim _{z \rightarrow \pm \infty}= \pm 1 \text {. }
$$

A função sigmoide, cujo gráfico tem a forma de $S$, é a forma mais comum de função de ativação (para a camada de saída da rede) utilizada na construção de redes neurais artificiais. Ela 
é definida como um função estritamente crescente que exibe um balanceamento adequado entre comportamento linear e não linear. Um bom exemplo de função sigmoide é a função logística

$$
\varphi(v)=\frac{1}{1+e^{-a v}},
$$

onde $a$ é uma constante que representa a declividade da curva da função $\varphi(v)$. Variando-se o parâmetro $a$, obtemos funções sigmoides com diferentes inclinações, como mostrado na figura 4.5 .

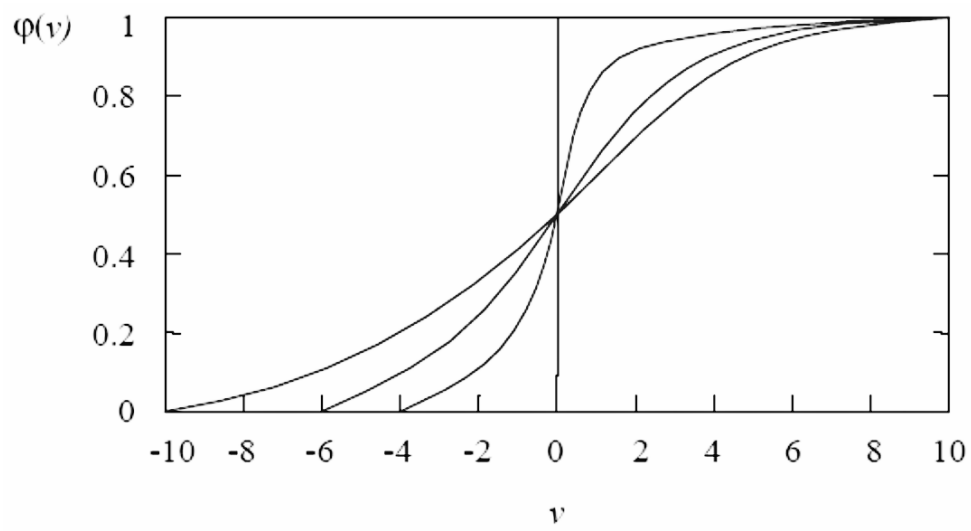

Figura 4.5: Gráfico da função sigmoide.

Vamos agora estender o modelo dado pela equação (4.6) para levar em conta a natureza temporal dos dados de entrada para armazenar memória. Uma maneira de modelar o comportamento temporal é representar cada neurônio como um circuito $R C$ cuja saída passa por um elemento não linear. Nestes termos, as entradas $x_{j}$ representam potenciais e os pesos sinápticos $w_{k j}$ denotam condutâncias. A equação de balanceamento para as correntes elétricas através do neurônio $k$ é dado pela lei de Kirchhoff:

$$
I_{C_{k}}+I_{R_{k}}=I_{\sin _{k}}+I_{a p l_{k}},
$$

onde $I_{C_{k}}$ é a corrente que passa pelo capacitor, $I_{R_{k}}$ a corrente que passa pelo resistor, $I_{a p l_{k}}$ é a corrente aplicada exteriormente, e $I_{s i n_{k}}$ é a corrente da sinapse, que é a soma de todas as correntes provenientes de todos os outros neurônios:

$$
I_{\text {sin }_{k}}(t)=\sum_{j=1}^{n} w_{k j} x_{j}(t),
$$

como podemos ver na Figura 4.6, a seguir.

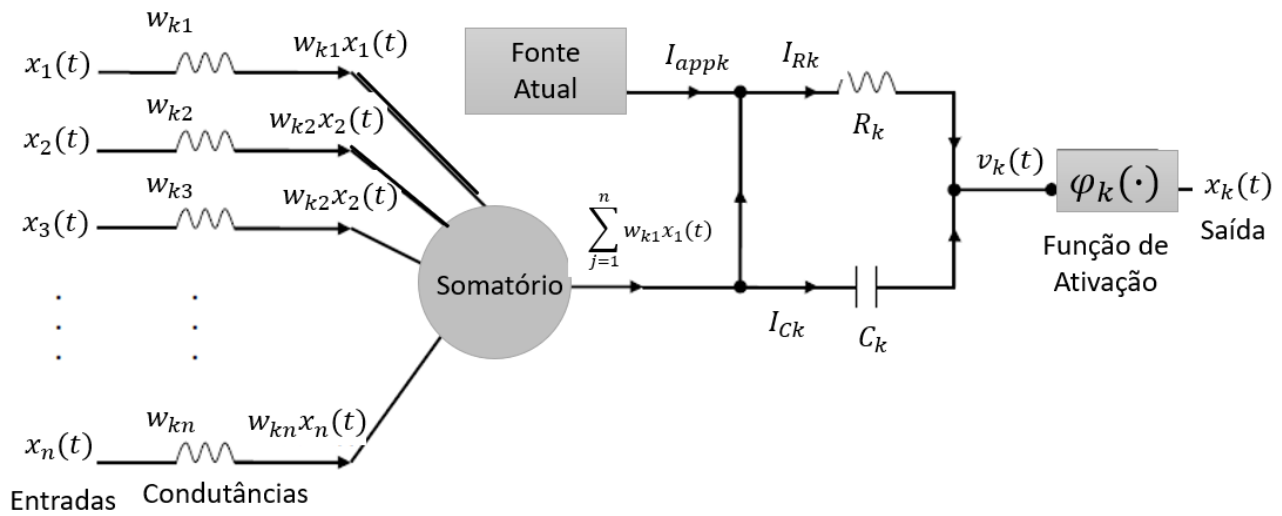

Figura 4.6: Modelo de uma rede neural de Hopfield. 
Agora, seja $v_{k}$ a voltagem do circuito $R C$ do neurônio $k$. Então, pela lei de Faraday, a corrente capacitiva é

$$
I_{C_{k}}=C_{k} \frac{d v_{k}(t)}{d t}
$$

onde $C_{k}$ é a capacitância do neurônio $k$. A corrente resistiva é dada pela Lei de Ohm,

$$
i_{R_{k}}=\frac{v_{k}(t)}{R_{k}},
$$

onde $R_{k}$ é a resistência do neurônio $k$. A saída do neurônio $j$ pode ser escrita como $x_{j}=\varphi_{j}\left(v_{j}(\mathrm{t})\right)$ e, substituindo as Equações 4.8, 4.9), 4.10) na Equação (4.7) obtemos uma dinâmica no modelo, definida como a rede neural de Hopfield:

$$
C_{k} \frac{d v_{k}}{d t}=-\frac{v_{k}(t)}{R_{k}}+\sum_{j=1}^{n} w_{k j} \varphi_{j}\left(v_{j}(t)\right)+I_{a p l_{k}}(t), k=1, \ldots, m
$$

Seja $v_{k}\left(t_{0}\right), k=1, \ldots, n$ as condições iniciais da rede de Hopfield. Desde que a função $\varphi(\cdot)$ é uma função limitada, todas as soluções são limitadas e o lado direito é de classe $C^{1}$. Assim, o problema de valor inicial dado pela equação diferencial ordinária (4.11) tem uma solução única definida em $\left[t_{0}, \infty\right]$, assegurado pelos teoremas de existência e unicidade das EDO's. Como interpretação física dos componentes do circuito $R C$, o capacitor e o resistor são paralelos para simular as características do atraso de tempo em neurônios biológicos. A resistência e a função de não-linearidade $\varphi_{j}(\cdot)$, que pode ser simulada por um amplificador operacional, são usadas para simular a sinapse e a característica não linear dos neurônios biológicos, respectivamente. Assim, usando uma notação simplificada para a Equação 4.11, as equações de estado e saída de Hopfield contínuo com $n$ neurônios são dadas como segue:

$$
\begin{aligned}
C_{k} \frac{d v_{k}}{d t} & =\sum_{j=1}^{n} w_{k j} x_{j}-\frac{v_{k}}{R_{k}}+I_{k} \\
v_{k} & =\frac{1}{\lambda} \varphi_{k}^{-1}\left(x_{k}\right) .
\end{aligned}
$$

onde $v_{k}(t)$ e $x_{k}(t)$ são a entrada e a saída do amplificador operacional (modelado pela função de ativação), respectivamente, para o $k$-ésimo neurônio no tempo $t$ e $w_{k j}$ a condutância entre o $k$-ésimo e $j$-ésimo neurônio. Em muitas aplicações da rede de Hopfield, é comum adicionar uma variável $\lambda \in \mathbb{R}$ que tem como objetivo controlar a taxa de aprendizagem na rede.

\subsection{Redes Neurais de Hopfield Fracionárias (FHNN)}

O conceito de uma rede neural de Hopfield em ordem fracionária foi desenvolvido por Boroomand e Menhaj [4, que propuseram substituir os clássicos capacitores por componentes denominado Capacitor Fracionário. O Capacitor fracionário, proposto por Nakagawa [4], é um novo componente físico, relacionado a um tipo de dielétrico, que pode ser descrito pelo operador de Caputo.

O capacitor fracionário pode ser definido como um capacitor generalizado por relação de ordem fracionária entre o terminal de tensão e a corrente que passa por ele. Em 1994, Westerlund [58. propôs um novo modelo de capacitor linear baseado na lei empírica de Curie que, para uma tensão de entrada geral $u(t)$, a corrente é :

$$
i(t)=F\left({ }_{0}^{\mathrm{C}} \mathrm{D}_{t}^{\alpha} u(t)\right)
$$

onde $0<\alpha<1$ e $F \in \mathbb{R}$ a capacitância fracionária. Desta ideia, podemos usar o capacitor generalizado na rede neural de Hopfield contínua ao invés dos capacitores comuns e assim definir 
uma nova rede neural, a rede neural de Hopfield com ordem fracionária (FHNN), descrita por equações diferenciais com ordem fracionária.

Definição 4.1 (rede neural de Hopfield de ordem fracionária) [4] Seja $0<\alpha<1$ e $v_{k}(t)$ uma função satisfazendo as hipóteses necessárias para aplicação da derivada de Caputo. As equações de estado e saída da rede neural de Hopfield de ordem fracionária são definidas como:

$$
\begin{gathered}
F_{k}\left({ }_{0}^{\mathrm{C}} \mathrm{D}_{t}^{\alpha} v_{k}(t)\right)=\sum_{j=1}^{p} w_{k j} x_{j}-\frac{v_{k}}{R_{k}}+I_{k} \\
v_{k}=\left(\frac{1}{\lambda}\right) \varphi_{k}^{-1}\left(x_{k}\right)
\end{gathered}
$$

onde $F_{k}$ é a fractância do neurônio $k$.

\subsection{Estabilidade de Hopfield}

Quando o número de neurônios da equação de Hopfield é muito grande, o modelo gera propriedades gerais com muitos graus de liberdade, não linearidade e dissipação. Um modelo assim gera estruturas de atratores complicadas, que pode gerar capacidades computacionais úteis. Com isso, torna-se fundamental a identificação de atratores em redes neurais como objetos computacionais.(por exemplo, a memória associativa). Para implementar esta ideia, devemos exercer controle sobre as localizações dos atratores no espaço de estados do sistema. Então teremos um algoritmo de aprendizagem que admite a forma de uma equação dinâmica não linear que manipula as localizações dos atratores para o propósito de codificar informação em uma forma desejada, ou aprender estruturas temporais de interesse. [20]

Para implementar uma tarefa computacional utilizando as propriedades coletivas de uma rede neural, usamos o conceito de minimização de energia da rede, que apresenta características de estabilidade dinâmica, pois há a garantia de que a rede convergirá para um dos atratores do sistema quando dado um padrão de entrada.

Vamos agora analisar a estabilidade das redes neurais de Hopfield através da função de energia de Lyapunov. Hopfield primeiramente definiu uma função, denominada como função de energia computacional:

$$
E(x)=-\frac{1}{2} \sum_{k=1}^{n} \sum_{j}^{n} w_{k j} x_{k} x_{j}-\sum_{k=1}^{n} x_{k} I_{k}+\frac{1}{\lambda} \sum_{k=1}^{n} \frac{1}{R_{k}} \int_{0}^{x_{k}} \varphi_{k}^{-1}(s) d s .
$$

Hopfield demonstra que caso nenhum neurônio da rede faça realimentação em si mesmo e a matriz de pesos seja simétrica $\left(w_{k j}=w_{j k}\right), \frac{d E}{d t} \leq 0$. Para isto, considere o modelo de Hopfield com $n$ neurônios

$$
C_{i} \frac{d v_{k}}{d t}=-\frac{v_{k}}{R_{k}}+\sum_{k=1}^{n} w_{k j} x_{k}+I_{k}, k=1,2, \ldots, n .
$$

Derivando a função de energia computacional em relação a $t$ ao longo das trajetórias da Equação (4.17), temos:

$$
\frac{d E}{d t}=\sum_{k=1}^{n} \frac{\partial E}{\partial x_{k}} \frac{d x_{k}}{d t} .
$$

Considerando $w_{k j}=w_{j k}$ e $w_{i i}=0$ e sabendo que $v_{k}=\varphi^{-1}\left(x_{k}\right)$, temos a partir da substituição da Equação (4.16) na Equação (4.18),

$$
\frac{d E}{d t}=\sum_{k=1}^{n}\left(-\sum_{j=1}^{n} w_{k j} x_{k}-I_{k}+\frac{v_{k}}{R_{k}}\right) \frac{d x_{k}}{d t} .
$$


Da Equação (4.17), obtemos

$$
\frac{d E}{d t}=\sum_{k=1}^{n}\left(-C_{k} \frac{d v_{k}}{d t} \frac{d x_{k}}{d t}\right)
$$

Como $\frac{d x_{k}}{d t}=\varphi_{k}^{\prime}\left(v_{k}\right) \frac{d v_{k}}{d t}$, temos da Equação 4.20

$$
\frac{d E}{d t}=-\sum_{k=1}^{n} C_{k}\left(\frac{d v_{k}}{d t}\right)^{2}\left(\varphi_{k}^{\prime}\left(v_{k}\right)\right)
$$

Devido ao fato da função de ativação ser monotonicamente crescente, $\varphi_{k}^{\prime}>0$, implica que

$$
\frac{d E}{d t}=-\sum_{k=1}^{n} C_{k}\left(\frac{d v_{k}}{d t}\right)^{2} \varphi_{k}^{\prime}\left(v_{k}\right) \leq 0
$$

Da equação definida na Equação (4.16), vemos que a função $E$ é limitada, e sua derivada é não positiva e se anula quando $\frac{d v_{k}}{d t}=0$, ou seja,

$$
-\frac{v_{k}}{R_{k}}+\sum_{j=1}^{n} w_{k j} x_{j}+I_{k}=0
$$

Assim, pelo Princípio da Invariância de LaSalle (Teorema 3.9), teremos um conjunto $\omega$-limite para $\frac{d E}{d t}=0$. Deste modo, a evolução temporal do modelo Hopfield contínuo descrito pelo sistema de equações diferenciais de primeira ordem não lineares, apresentado na Eq. (4.11) representa uma trajetória no espaço de estados que busca os mínimos da função de energia $E$ e se mantém em tais pontos fixos. Deste modo, a rede neural de Hopfield possui atratores, que podem ser interpretados como uma evolução de um padrão distorcido em direção a um padrão desejado, o equilíbrio. Isso leva à compreensão da RNH como um método associativo. [20]

Vamos analisar agora a estabilidade de uma FHNN através da função de energia de Lyapunov de redes neurais. Da equação da FHNN definida na equação 4.15, a função de energia para esta rede é

$$
E=-\frac{1}{2} \sum_{k=1}^{n} \sum_{j}^{n} w_{k j} x_{k} x_{j}-\sum_{k=1}^{n} x_{k} I_{k}+\frac{1}{\lambda} \sum_{k=1}^{n} \frac{1}{R_{k}} \int_{0}^{x_{k}} \varphi_{k}^{-1}(s) d s
$$

Para mostrar a estabilidade da rede neural proposta, precisamos de alguma forma generalizar a ideia do operador gradiente para ordem fracionária:

Definição 4.2 O gradiente fracionário de uma função escalar com respeito à variável $x=\left(x_{1}, x_{2}, \ldots, x_{n}\right)$ é definido por $\nabla^{(\alpha)} f(x)$, onde $\nabla^{(\alpha)}$ representa o operador de gradiente fracionário. O gradiente fracionário de $f(x)$ contém o campo vetorial cujas componentes são a derivada de Caputo da função, como segue:

$$
\nabla_{x}^{(\alpha)} f(x)=\left({ }^{\mathrm{C}} \mathrm{D}_{x_{1}}^{\alpha} f(x),{ }^{\mathrm{C}} \mathrm{D}_{x_{2}}^{\alpha} f(x), \ldots,{ }^{\mathrm{C}} \mathrm{D}_{x_{n}}^{\alpha} f(x)\right)
$$

Teorema 4.1 [4] Para a FHNN definida na Equação (4.15), se $\varphi_{k}^{-1}\left(x_{k}\right)$ é uma função contínua monótona crescente, e $F_{k}>0$, então a função de energia da FHNN tem gradiente fracionário definido negativo e os seguintes resultados são assegurados:

$$
\nabla^{(\alpha)} E \leq 0, \quad \text { e } \quad \nabla^{(\alpha)} E=0 \quad \text { se e somente se }{ }^{\mathrm{C}} \mathrm{D}_{t}^{\alpha} x_{k}=0, k=1,2, \ldots, n
$$

Demonstração. Vamos começar definindo uma função $y(x), x=x(t)$, que satisfaz as hipóteses requeridas para a integração e derivação de ordens fracionárias. Neste caso, aplicando a derivada 
de Caputo, temos:

$$
\begin{aligned}
{ }_{0}^{\mathrm{C}} \mathrm{D}_{t}^{\alpha} y(x)=J_{t}^{1-\alpha} y^{\prime}(x)=J_{t}^{1-\alpha}\left(\frac{\partial y}{\partial x} x^{\prime}(t)\right) & =\frac{1}{\Gamma(1-\alpha)} \int_{0}^{t}(t-\tau)^{-\alpha} \frac{\partial y}{\partial x} x^{\prime}(\tau) d \tau \\
& =\frac{\partial y}{\partial x} \frac{1}{\Gamma(1-\alpha)} \int_{0}^{t}(t-\tau)^{-\alpha} x^{\prime}(\tau) d \tau .
\end{aligned}
$$

Como

$$
{ }_{0}^{\mathrm{C}} \mathrm{D}_{t}^{\alpha} x(t)=J_{t}^{1-\alpha} x^{\prime}(t)=\frac{1}{\Gamma(1-\alpha)} \int_{0}^{t}(t-\tau)^{-\alpha} x^{\prime}(\tau) d \tau
$$

temos então que

$$
{ }_{0}^{\mathrm{C}} \mathrm{D}_{t}^{\alpha} y(x)={ }_{0}^{\mathrm{C}} \mathrm{D}_{t}^{\alpha} x(t) \frac{\partial y}{\partial x}
$$

que é um resultado análogo ao cálculo com ordem inteira. Tendo o resultado da equação (4.26) em mente, derivando o primeiro e segundo termos da função de energia (4.23), obtemos como resultado

$$
\begin{aligned}
{ }^{\mathrm{C}} \mathrm{D}_{t}^{\alpha}\left(-\frac{1}{2} \sum_{k=1}^{n} \sum_{j=1}^{n} w_{i j} x_{j} x_{k}\right) & ={ }^{\mathrm{C}} \mathrm{D}_{t}^{\alpha}\left(-\frac{1}{2} X^{T} W X\right) \\
& =-\frac{1}{2} \frac{\partial}{\partial x}\left(X^{T} W X\right){ }^{\mathrm{C}} \mathrm{D}_{t}^{\alpha} X=-\sum_{k=1}^{n}{ }^{\mathrm{C}} \mathrm{D}_{t}^{\alpha} x_{k} \sum_{j=1}^{n} w_{k j} x_{j}, \\
{ }^{\mathrm{C}} \mathrm{D}_{t}^{\alpha}\left(-\sum_{k}^{n} x_{k} I_{k}\right) & =-\sum_{k}^{n} I_{k}{ }^{\mathrm{C}} \mathrm{D}_{t}^{\alpha} x_{k} .
\end{aligned}
$$

Agora, da definição (4.2) e da equação (4.26) e procedendo de maneira análoga ao que foi feito anteriormente, temos que o gradiente da função de energia da rede neural fracionária de Hopfield será:

$$
\begin{aligned}
\nabla_{t}^{\alpha} E & =\sum_{k=1}^{n} \frac{\partial E}{\partial x_{k}}{ }^{\mathrm{C}} \mathrm{D}_{t}^{\alpha} x_{k}= \\
& =-\sum_{k=1}^{n}{ }^{\mathrm{C}} \mathrm{D}_{t}^{\alpha} x_{k} \sum_{j=1}^{n} w_{k j} x_{j}-\sum_{k}^{n} I_{k}{ }^{\mathrm{C}} \mathrm{D}_{t}^{\alpha} x_{k}+\frac{1}{\lambda} \sum_{k=1}^{n} \frac{1}{R_{k}}\left({ }^{\mathrm{C}} \mathrm{D}_{t}^{\alpha} x_{k}\right) \frac{\partial}{\partial x_{k}} \int_{0}^{x_{k}} v_{k}(s) d s \\
& =-\sum_{k}^{n}{ }^{\mathrm{C}} \mathrm{D}_{t}^{\alpha}\left(\sum_{j=1}^{n} w_{k j} x_{k}+I_{k}-\frac{v_{k}}{R_{k}}\right) \\
& =-\sum_{k}^{n}{ }^{\mathrm{C}} \mathrm{D}_{t}^{\alpha} x_{k} F_{k}{ }^{\mathrm{C}} \mathrm{D}_{t}^{\alpha} v_{k} \\
& =-\sum_{k=1}^{n}\left({ }^{\mathrm{C}} \mathrm{D}_{t}^{\alpha} x_{k}\right)^{2} F_{k} \frac{\partial}{\partial x_{k}} \varphi_{k}^{-1}\left(x_{k}\right)
\end{aligned}
$$

Tendo em vista que $\varphi_{k}^{-1}$ é uma função contínua monotonicamente crescente e $F_{k}>0$, temos que o resultado 4.25 é válido.

\subsection{Algumas aplicações das Redes Neurais de Hopfield fracionárias}

A principal vantagem de utilizar modelos com ordem fracionária, em comparação com modelos de ordem inteira, é que as derivadas fracionárias fornecem uma excelente ferramenta para a descrição da memória e propriedades hereditárias de vários processos. De fato, conforme vimos, os sistemas de ordem fracionária têm memória infinita. Levando em conta este atributo, é fácil ver que 
a incorporação de um termo de memória (na forma de uma derivada fracionária) em um modelo de rede neural que utiliza memória associativa é uma melhoria bastante importante.

Hopfield e Tank [23], demonstraram o poder computacional e a velocidade das redes neurais na solução rápida em problemas de otimização. No entanto, uma solução ótima global não é garantida porque este problema de otimização pode ter muitos ótimos locais. Reynold et al [9] indicam como aplicar uma rede Hopfield ao problema de identificação linear de sistemas. A identificação de sistemas tem como objetivo derivar um modelo matemático a partir de dados observados do sistema. Dependendo da informação prévia disponível, há várias abordagens que podem ser utilizadas. Medindo entradas, variáveis de estado e derivadas temporais de variáveis de estado, eles apresentaram um procedimento para programar uma rede de Hopfield. Os estados dos neurônios dessa rede convergem para os valores dos parâmetros do sistema, que devem ser identificados. Em [4, o autor usa a rede de Hopfield fracionária

$$
{ }^{\mathrm{C}} \mathrm{D}_{t}^{\alpha} v_{k}(t)=\sum_{k=0}^{n} w_{k j} \frac{1}{2}\left(1+\tanh \left(\lambda \frac{v_{k}(t)}{v_{0}}\right)\right)+I_{k}
$$

para o procedimento de Hopfield e Tank, demonstrando sua eficácia ao mostrar que tal rede também converge para os valores de parâmetros do sistema considerado.

Além da resolução de probemas de otimização, uma das principais aplicações da rede neural de Hopfield é a recuperação de padrões mesmo em dados corrompidos. Uma rede neural de Hopfield que associa padrões funcionaria da seguinte forma: 1) Fase de armazenamento, onde os padrões estão sendo armazenados pela Rede Neural; 2) Fase de recuperação,onde os padrões estão sendo recuperados em resposta a um padrão distorcido apresentado pela rede.

Para que a rede neural recupere padrões, é necessário uma regra de aprendizagem. Uma das principais regras é a regra de Hebb. Trata-se da primeira regra de aprendizado para redes neurais artificiais, proposta por Donald Hebb em 1949 [?]. Sua premissa é que se os dois neurônios, participantes de uma sinapse, têm ativação simultânea, então a força da conexão entre eles deve ser seletivamente aumentada. ou seja,

$$
\Delta w_{k j}(n)=\eta y_{k}(n) x_{l}(n),
$$

onde $\eta$ é uma constante positiva que determina a taxa de aprendizagem, $x_{j}$ o sinal pré sináptico, $y_{k}$ o sinal pós sináptico, $w_{k j}$ o peso sináptico e $n$ o número de iteração do algoritmo. $\mathrm{O}$ aprendizado de Hebb é análogo ao aprendizado humano, onde acredita-se que o aprendizado está relacionado a alterações na eficácia das sinapses que conectam os neurônios

No artigo de J. Hu et al [24] (2012), é feita uma comparação entre os modelos de Hopfield de ordens fracionária e inteira para recuperação de padrões. Com o objetivo de verificar o desempenho da FHNN foram realizados experimentos de reconhecimento usando os sinais numéricos 09 . Os dados de treinamento incluem 1 grupo de sinal numérico impresso; os dados de teste incluem 12 grupos de sinal numérico impresso, cada grupo tem 10 sinais numéricos: 0 9, que são corrompidos por ruído aleatório de vários níveis. No experimento, foi comparado o desempenho entre as redes neurais Hopfield de ordem fracionária e as redes neurais Hopfield padrão através de números impressos corrompidos por ruído. Como conclusão, percebe-se que para os três conjuntos de amostras, a FHNN obteve uma taxa de precisão maior que as redes neurais de Hopfield de ordem inteira. Assim, os resultados da simulação, em comparação com a HNN, mostraram algumas vantagens destacadas na rede de Hopfield de ordem fracionária, que contém uma maior capacidade de memória. 


\section{Capítulo 5}

\section{Estabilidade de Hopfield em Redes Neurais Fracionárias}

No Capítulo 4 introduzimos as neurais de Hopfield e mostramos, a partir da derivada de sua função de energia, que esta rede busca pontos de equilíbrio (mínimos da função de energia $E$ ). Autores como em [34] argumentam que este é um tipo diferente da estabilidade de Lyapunov, denominando-a como estabilidade no sentido de Hopfield. Isso acontece pois o princípio de invariância de LaSalle não traz conclusões a respeito da estabilidade no sentido de Lyapunov. Os seguintes argumentos favoráveis a esta conclusão são [47]:

1. A função de energia não é necessariamente uma função de Lyapunov, pois não é garantido que $E>0$, conforme a definição exige;

2. Ao analisar a estabilidade de uma sistema dinâmico no sentido de Lyapunov, deve-se conhecer o ponto de equilíbrio, o que não ocorre na estabilidade no sentido de Hopfield, que procura por esses pontos;

3. Não se pode concluir que o ponto de equilíbrio seja o mínimo de $E$ como na estabilidade de Lyapunov, pois na estabilidade de Hopfield poderia ser um ponto de inflexão, pois a derivada de primeira ordem igual a zero é apenas uma condição necessária para obtermos o valor mínimo.

Devido a esses fatores, torna-se importante um estudo da estabilidade da rede neural de Hopfield. A estabilidade dos pontos de equilíbrio de uma rede neural dinâmica é uma das propriedades mais básicas e importantes para muitas aplicações destas redes na engenharia. É importante notar que nos referimos à estabilidade no sentido de Lyapunov [28].

Conforme define Haykin [20], o estudo das redes neurais vistas como sistemas dinâmicos não lineares, com particular ênfase no problema da estabilidade, é referido como neurodinâmica. A estabilidade das redes neurais tem as mesmas complexidades do estudo da estabilidade de sistemas não lineares.

A presença de estabilidade sempre implica alguma forma de coordenação entre as partes individuais do sistema. Com relação às FHNN, é necessário que haja uma solução computável bem definida para todos os possíveis estados iniciais. Do ponto de vista matemático, a análise da estabilidade de um ponto de equilíbrio único é muito necessária e valiosa [61].

Motivado pelos argumentos apresentados, neste capítulo estaremos preocupados na neurodinâmica das FHNNs. Aplicaremos os conceitos já estudados de estabilidade no sentido de Lyapunov e a estabilidade de Mittag-Leffler para sistemas de ordem fracionária, com o objetivo de criar critérios de estabilidade particulares à essas redes neurais. 


\subsection{Um exemplo de critério de estabilidade para as Redes de Hop- field}

Muitas condições locais e globais para analisar a estabilidade de redes neurais dinâmicas foram introduzidas nas décadas de 80 e 90, e derivadas usando diferentes abordagens de análise não linear como as análises de Cohen e Grossberg [10, Guez et al. [18, Kelly [27] e Matsuoka [40, para uma classe geral de redes neurais dinâmicas de tempo contínuo, que podem ser consideradas como versões generalizadas das bem conhecidas redes neurais de Hopfield. Em 2000, [17] estabelece um conjunto de resultados para a estabilidade global das RNH's que são menos conservadores e mais gerais do que as anteriormente estudadas. Vamos considerar o sistema dado pela Equação 4.12 $\operatorname{com} \lambda=1$,

$$
\begin{aligned}
C_{k} \frac{d v_{k}}{d t} & =\sum_{j=1}^{m} w_{k j} x_{j}-\frac{v_{k}}{R_{k}}+I_{k} \\
x_{k} & =\varphi_{k}\left(v_{k}\right) \cdot k=1, \ldots, n .
\end{aligned}
$$

Aqui não admitimos a simetria da matriz $w_{k j}$, somente que $\varphi \in C^{1}, \varphi^{\prime}$ é inversível (aqui denotaremos $\left.v_{k}=\varphi_{k}^{-1}\left(x_{k}\right):=G\left(x_{k}\right)\right)$, e satisfaz $0<m_{k} \leq \varphi_{k}^{\prime} \leq M_{k}<\infty$ uniformemente sobre o domínio de $\varphi$. Segue do teorema da função inversa que

$$
\frac{1}{M_{k}} \leq G_{k}^{\prime}=\left(\varphi_{k}^{-1}\left(x_{k}\right)\right)^{\prime}=\left(\varphi_{k}^{\prime}\left(x_{k}\right)\right)^{-1} \leq \frac{1}{m_{k}}, k=1, \ldots, n .
$$

Como $v_{k}:=G\left(x_{k}\right)$, podemos reescrever a Equação 5.1 como:

$$
C_{k} G^{\prime}\left(x_{k}\right) \frac{d x_{k}}{d t}=\sum_{j=1}^{n} w_{k j} x_{j}-\frac{G_{k}}{R_{k}}+I_{k} .
$$

Se $x^{*}=\left(x_{1}, \ldots, x_{n}\right)^{T}$ é um ponto de equilíbrio da Equação (5.1), então podemos reescrever a Equação (5.4) como

$$
C_{k} G^{\prime}\left(x_{k}-x_{k}^{*}\right) \frac{d x_{k}}{d t}=\sum_{j=1}^{n} w_{k j}\left(x_{j}-x_{j}^{*}\right)-\frac{G_{k}\left(x_{k}\right)-G_{k}\left(x_{k}^{*}\right)}{R_{k}} .
$$

Teorema 5.1 [17] Se as condições 1 ou 2 são satisfeitas, então o ponto de equilíbrio do sistema (5.1) é globalmente assintoticamente estável.

1. Existem constantes $p_{k}>0, k=1, \ldots, n, j=1, \ldots n$ tal que :

$$
p_{j}\left(w_{j j}-\frac{1}{R_{j} M_{j}}\right)+\sum_{k=1, k \neq j}^{n} p_{k}\left|w_{k j}\right|<0
$$

2. A seguinte desigualdade é satisfeita

$$
w_{k k}-\frac{1}{R_{k} M_{k}}+\sum_{j=1, j \neq k}^{n}\left|w_{k j}\right|<0
$$

Demonstração. Vamos supor que a condição 1 é assegurada. Usaremos a seguinte função de Lyapunov

$$
V(x)=\sum_{k=1}^{n} p_{k} C_{k}\left|\int_{x_{k}^{*}}^{x_{k}} G_{k}^{\prime}\left(\chi_{k}\right) d \chi_{k}\right| .
$$


Obviamente, como $p_{k}$ e $C_{k}$ são constantes positivas, temos que $V(x) \geq 0$. Desde que

$$
\left|\int_{x_{k}^{*}}^{+\infty} G_{k}^{\prime}\left(\chi_{k}\right) d \chi_{k}\right| \geq\left|\int_{x_{k}^{*}}^{+\infty} \frac{1}{M_{k}} d \chi_{k}\right|=+\infty, k=1, \ldots, n,
$$

então $V(x) \rightarrow \infty$ quando $\|x\| \rightarrow \infty$. Calculando a derivada de Dini superior da função $V(x)$ ao longo da trajetória da Equação (5.5), temos que, usando a regra de Leibniz:

$$
\begin{aligned}
D^{+} V(x) & =\sum_{k=1}^{n} p_{k} C_{k}\left(\frac{d x_{k}}{d t} G_{k}^{\prime}\left(x_{k}\right)-\frac{d x_{k}^{*}}{d t} G_{k}^{\prime}\left(x_{k}\right)+\int_{x_{k}^{*}}^{x_{k}(t)} \frac{\partial}{\partial t} G_{k}^{\prime}\left(\chi_{k}\right) d \chi_{k}\right) \operatorname{sgn}\left(x_{k}-x_{k}^{*}\right) \\
& =\sum_{k=1}^{n} p_{k} C_{k}\left(\frac{d x_{k}}{d t} G_{k}^{\prime}\left(x_{k}\right)\right) \operatorname{sgn}\left(x_{k}-x_{k}^{*}\right),
\end{aligned}
$$

onde $\operatorname{sgn}(x)$ é a função sinal. Da fórmula de Taylor, temos que

$$
G_{k}\left(x_{k}\right)=G_{k}\left(x_{k}^{*}\right)+G_{k}^{\prime}\left(\eta_{k}\right)\left(x_{k}-x_{k}^{*}\right),
$$

com $\eta_{k}$ estando entre $x_{k}$ e $x_{k}^{*}$ e

$$
\left(G_{k}\left(x_{k}\right)-G_{k}\left(x_{k}^{*}\right)\right) \operatorname{sgn}\left(x_{k}-x_{k}\right)=G_{k}^{\prime}\left(\eta_{k}\right)\left|x_{k}-x_{k}^{*}\right| .
$$

Utilizando as Equações 5.7 e 5.5 em $D^{+} V(x)$, obtemos

$$
\begin{aligned}
D^{+} V(x) & =\sum_{k=1}^{n} p_{k}\left(\sum_{j=1}^{n} w_{k j} x_{k}-\frac{G_{k}\left(x_{k}\right)}{R_{k}}\right) \operatorname{sgn}\left(x_{k}-x_{k}^{*}\right) \\
& \leq \sum_{k=1}^{n} p_{k}\left(\sum_{j=1}^{n} w_{k j}\left(x_{k}-x_{k}^{*}\right)-\frac{G_{k}\left(x_{k}\right)-G_{k}\left(x_{k}^{*}\right)}{R_{k}}\right) \operatorname{sgn}\left(x_{k}-x_{k}^{*}\right) \\
& \leq \sum_{j=1}^{n}\left(p_{j} w_{j j}+\sum_{k=1, k \neq j}^{n} p_{k}\left|w_{k j}\right|\right)\left|x_{k}-x_{k}^{*}\right|-\sum_{j=1}^{n} \frac{p_{j}}{R_{j}} G_{j}^{\prime}\left(\eta_{j}\right)\left|x_{j}-x_{j}^{*}\right| \\
& =\sum_{j=1}^{n}\left[p_{j}\left(w_{j j}-\frac{1}{R_{j} M_{j}}\right)+\sum_{k=1, k \neq j}^{n} p_{k}\left|w_{k j}\right|\right]\left|x_{j}-x_{j}^{*}\right|+\sum_{j=1}^{n} \frac{p_{j}}{R_{j}}\left(\frac{1}{M_{j}}-G_{j}^{\prime}\left(\eta_{j}\right)\right)\left|x_{j}-x_{j}^{*}\right| \\
& \leq \sum_{j=1}^{n}\left[p_{j}\left(w_{j j}-\frac{1}{R_{j} M_{j}}\right)+\sum_{k=1, k \neq j}^{n} p_{k}\left|w_{k j}\right|\right]\left|x_{j}-x_{j}^{*}\right| \\
& <0, \text { se } x \neq x^{*} .
\end{aligned}
$$

Suponha agora que a condição 2) esteja assegurada. Neste caso, vamos usar a função de Lyapunov

$$
V(x)=\max _{1 \leq j \leq n}\left|x_{j}-x_{j}^{*}\right| \doteq\left|x_{i}-x_{i}^{*}\right|,
$$


e de maneira similar ao que foi feito anteriormente,

$$
\begin{aligned}
D^{+} V(x)= & \frac{d x_{i}}{d t} \operatorname{sgn}\left(x_{i}-x^{*}\right) \\
& \left.\leq \frac{1}{C_{i} G_{i}^{\prime}\left(x_{i}\right)}\left[\left(w_{i i}+\sum_{j=1, j \neq i} \mid w_{i j}\right]\right)\left|x_{i}-x_{i}^{*}\right|-\frac{1}{R_{i}} G_{i}^{\prime}\left(\eta_{i}\right)\left|x_{i}-x^{*}\right|\right] \\
& =\frac{1}{C_{k} G_{k}^{\prime}\left(x_{k}\right)}\left[\left(w_{i i}-\frac{1}{R_{i} M_{i}}+\sum_{j=1, j \neq i}\left|w_{i j}\right|\right)\left|x_{i}-x_{i}^{*}\right|+\frac{1}{R_{i}}\left(\frac{1}{M_{i}}-G_{i}^{\prime}\left(\eta_{i}\right)\right)\left|x_{i}-x_{i}^{*}\right|\right] \\
& \leq \frac{1}{C_{i} G_{i}^{\prime}\left(x_{i}\right.}\left(w_{i i}-\frac{1}{R_{i} M_{i}}+\sum_{j=1, j \neq i}^{n}\left|w_{i j}\right|\right)\left|v_{i}-v_{i}^{*}\right|
\end{aligned}
$$

$<0$ se $x \neq x^{*}$,

o que verifica a hipótese do teorema.

Exemplo 5.1 Vamos considerar a seguinte rede de Hopfield

$$
\begin{aligned}
& \dot{v}_{1}(t)=-6 v_{1}(t)+2 \operatorname{sen}\left(v_{1}(t)\right)+\operatorname{sen}\left(v_{2}(t)\right)-3 \operatorname{sen}\left(v_{2}(t)\right)+I_{1} \\
& \dot{v}_{2}(t)=-5 v_{2}(t)-2 \operatorname{sen}\left(v_{1}(t)\right)-0.4 \operatorname{sen}\left(v_{2}(t)\right)+\operatorname{sen}\left(v_{3}(t)\right)+I_{2} \\
& \dot{v}_{3}(t)=-8 v_{3}(t)+\operatorname{sen}\left(v_{1}(t)-2.5 \operatorname{sen}\left(v_{2}(t)\right)+3.5 \operatorname{sen}\left(v_{3}(t)\right)+I_{3} .\right.
\end{aligned}
$$

Neste caso temos uma rede de Hopfield com $n=3$, satisfazendo as condições de existência $e$ unicidade, já que a função sen $(v(t))$ é Lipschitiziana, $v(t)=\left(v_{1}(t), v_{2}(t), v_{3}(t)\right)^{T}, x_{j}=\varphi_{j}\left(v_{j}(t)\right)=$ $\operatorname{sen}\left(v_{j}(t)\right), j=1,2,3, \frac{1}{R_{1}}=6, \frac{1}{R_{2}}=5, \frac{1}{R_{3}}=8, I_{1}=\pi-4, I_{2}=2, I_{3}=3-4 \pi e$

$$
w_{j k}=\left[\begin{array}{ccc}
2 & 1 & -3 \\
-2 & -0.4 & 1 \\
1 & -2.5 & 3.5
\end{array}\right] .
$$

Aplicando os critérios do teorema (5.1), podemos escolher $M_{j}=1$, já que $\operatorname{sen}\left(v_{j}(t)\right)^{\prime} \leq 1$ e com isso vemos facilmente que as duas condições são satisfeitas, demonstrando que o único ponto de equilíbrio do sistema, $v_{t}^{*}=\left(\frac{\pi}{6}, 0 . \frac{\pi}{2}\right)^{T}$, é globalmente estável. Conforme observado na Figura 5.1 , com a condição inicial $(4,4,4)^{T}$, a solução da rede de Hopfield de fato converge para o ponto de equilíbrio do sistema.

\subsection{Estabilidade de Mittag-Leffler para FHNNs}

Analisaremos agora a estabilidade para a FHNN dada pelo sistema 4.15. Usando a método descrito pelo Teorema (3.11), devemos encontrar uma função que satisfaça as condições (3.53) e (3.54). Mas, conforme comentado em [61], é muito difícil encontrar funções que satisfaçam tais condições, em razão de que métodos de ordem inteira para construir funções de Lyapunov não são efetivos para sistemas de ordem fracionária. Daí torna-se necessário enfraquecê-las de modo a tornar viável a aplicação de meios que facilitem o estudo da estabilidade de RNHFs. Um modo de enfraquecer as condições do Teorema (3.11) é usar o conceito de propriedade quase sempre assegurada. Suponha que temos um espaço de medidas $(X, \Sigma, \mu)$, onde $X$ é um conjunto não vazio, $\Sigma$ é uma $\sigma$-álgebra (álgebra de Borel) do conjunto $X$ e $\mu$ é uma medida em $(X, \Sigma)$. Assim, temos que:

Definição 5.1 Dado o espaço de medidas $(X, \Sigma, \mu)$, uma propriedade $P$ diz ser quase sempre assegurada em $X$ se existe um conjunto $C \in \Sigma \operatorname{com} \mu(C)=0$ e para $x \in X$ Cé assegurada a propriedade $P$. 


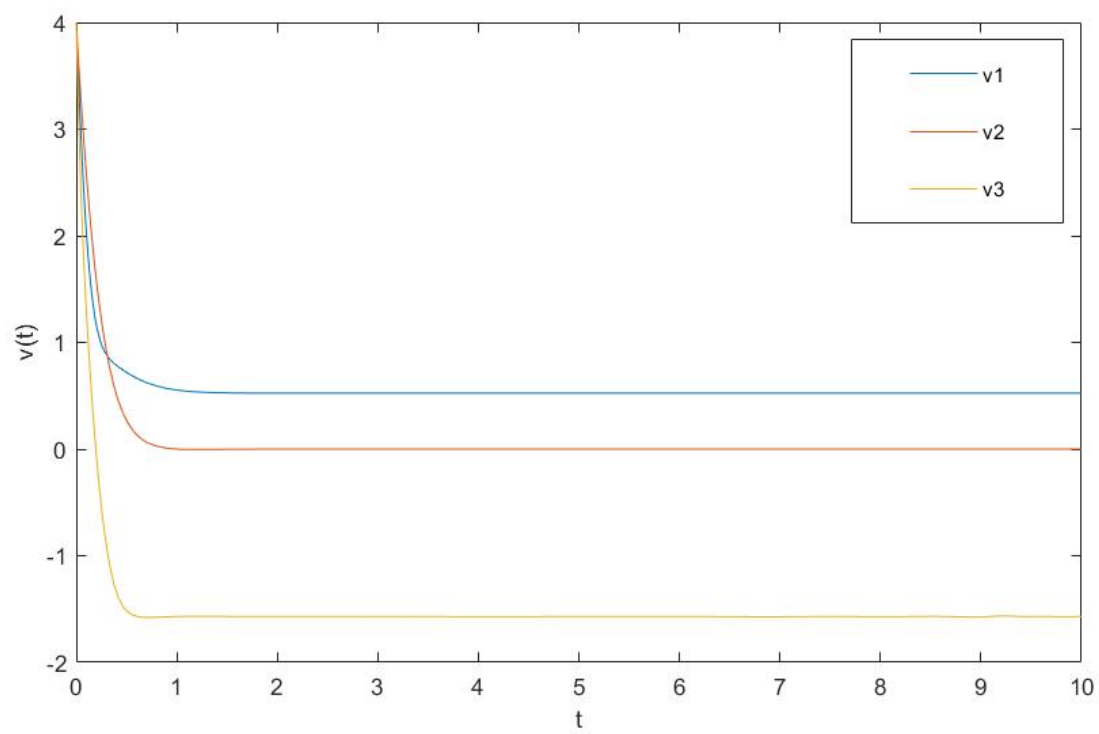

Figura 5.1: Solução da rede de Hopfield do exemplo 5.1 com condição inicial $(4,4,4)^{T}$.

A Definição 5.1 basicamente no diz que uma propriedade é válida em quase todo lugar no conjunto $\mathrm{D}$ se os únicos pontos em que ela não é válida constituem um conjunto de medidas nula. O conceito de medida nula indica probabilidade zero na teoria de probabilidades. Um subconjunto contável do $\mathbb{R}^{n}$ sempre terá medida nula. Por exemplo, dada a função

$$
\left\{\begin{array}{c}
x \text { if } x \in \mathbb{R} \backslash \mathbb{Q} \\
0 \text { if } x \in \mathbb{Q}
\end{array}\right.
$$

tendo em mente que $\mathbb{Q}$ é contável, a propriedade "estritamente crescente"é quase sempre assegurada em $\mathbb{R}$.

O teorema a seguir modifica o Teorema (3.11) de forma a enfraquecer a desigualdade (3.54) .

Teorema 5.2 [33] [Método direto de Lyapunov para sistemas fracionários] Seja $x=0$ o ponto de equilíbrio do sistema (3.42) e $D \in \Re^{n}$ um dominio contendo a origem. Seja $V(t, x(t)):[0, \infty] \times B \rightarrow$ $\Re$ uma função continuamente diferenciável e localmente Lipschitziana com respeito a $x$ tal que

$$
\begin{aligned}
& \alpha_{1}\|x\|^{a} \leq V(t, x) \leq \alpha_{2}\|x\|^{a b}, \\
& { }_{0}^{C} D_{t}^{\alpha} V\left(t^{+}, x\left(t^{+}\right)\right) \leq-\alpha_{3}\|x\|^{a b} \text { (Quase sempre assegurado) }
\end{aligned}
$$

onde $t \geq 0, \alpha \in(0,1), x \in B, \alpha_{1}, \alpha_{2}, \alpha_{3}, a$ e $b$ são contantes arbitrárias, $\dot{V}$ é contínua por partes e $V\left(t^{+}, x\left(t^{+}\right):=\lim _{\tau \rightarrow t^{+}} V(\tau, x(\tau))\right.$ positiva. Então o ponto de equilíbrio $x=0$ é globalmente Mittag-Leffler estável.

Com o objetivo de construir uma função de Lyapunov apropriada, o Teorema (5.3) sobre uma desigualdade para a derivada de Caputo será fundamental.

Teorema 5.3 Se $h(t) \in C^{1}([0,+\infty))$, a seguinte desigualdade é quase sempre assegurada em todo lugar,

$$
{ }^{\mathrm{C}} \mathrm{D}_{t}^{\alpha}\left|h\left(t^{+}\right)\right| \leq \operatorname{sgn}(h(t)){ }^{\mathrm{C}} \mathrm{D}_{t}^{\alpha} h(t), 0<\alpha<1,
$$

onde, $h\left(t^{+}\right):=\lim _{\tau \rightarrow t^{+}} h(\tau)$ e $\operatorname{sgn}(h(t))$ é o sinal da função $h(t)$.

Demonstração. Como $h(t)$ é continuamente diferenciável, $|h(t)|$ também é diferenciável, exceto no conjunto $\phi=\left\{t \mid h(t)=0, h^{\prime}(t) \neq 0\right\}$. Assim $\frac{d|h(t)|}{d t}$ é contínua por partes e $\lim _{\tau \rightarrow t} \frac{d|h(\tau)|}{d \tau}$ existe 
para qualquer $t \in[0, \infty)$. Isso demonstra que o conjunto $\phi$ com relação à função $|h(t)|$ tem medida nula. A função $|h(t)|$ é quase sempre diferenciável, como por exemplo, na Figura 5.2. De maneira formal, para qualquer $T \in[0, \infty)$, o intervalo $[0, T]$ pode ser dividido em $n \in \mathbb{N}$ partes com o mesmo comprimento. Quando $n$ tende ao infinito, cada intervalo tem no máximo um ponto pertencente ao conjunto de medida nula $\phi$, caso contrário existiriam dois pontos $t_{1}$ e $t_{2} \in \phi$ com $\lim _{t_{2}-t_{1} \rightarrow 0} \frac{h\left(t_{2}\right)-h\left(t_{1}\right)}{t_{2}-t_{1}}=0$, o que seria absurdo, já que $h^{\prime}(t) \neq 0$. Como $\phi$ é um conjunto contável com medida nula, $|h(t)|$ é quase sempre diferenciável. Devemos mostrar agora que a desigualdade (5.11)

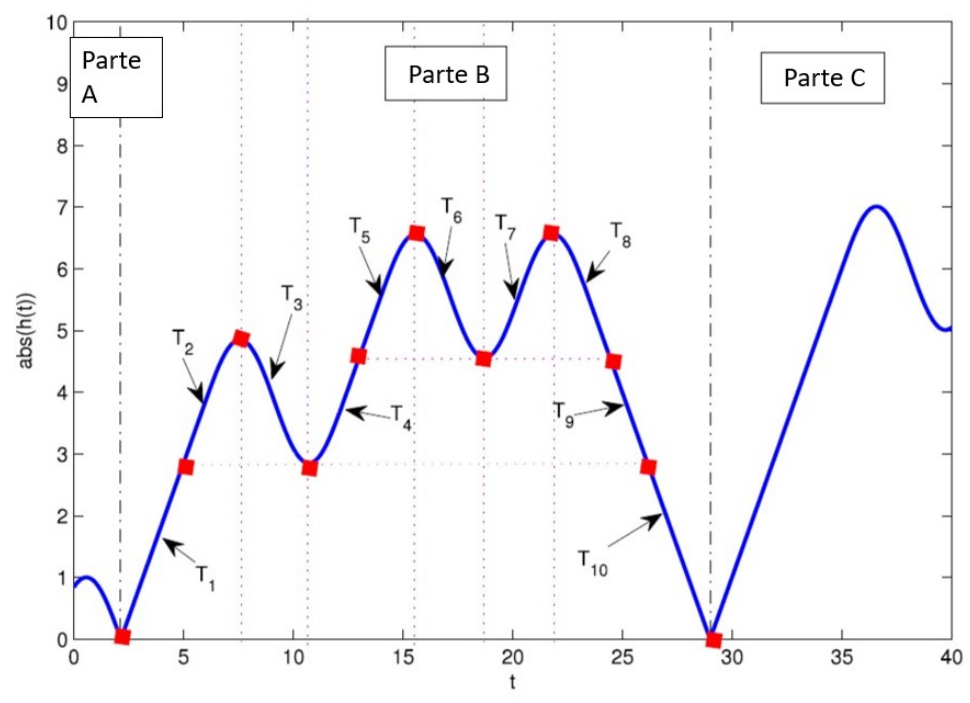

Figura 5.2: Exemplo de uma trajetória de $|h(t)|$. [61].

é assegurada exceto no conjunto $\phi$. Sem perda de generalidade, podemos descrever a trajetória de $|h(t)|$ como na Figura 5.2. Por simplicidade, vamos começar supondo um trajetória como na Figura 5.3 Neste caso temos apenas um ponto extremo em cada parte (pontos $P_{2}$ e $P_{4}$ ). A trajetória é formada em três partes por $\min \{t, t \in \phi\}$ e $\max \{t, t \in \phi\}\left(\right.$ pontos $P_{3}$ e $P_{5}$ ). Cada parte é dividida em dois intervalos onde o extremo é um ponto extremo da função como $T b_{1}$ e $T b_{2}$ na parte $\mathrm{A}$. $\mathrm{O}$ ponto $P_{1}$ é a condição inicial $h(0)$. Cada ponto tem como coordenadas $P_{a}=\left(X p_{a}, Y p_{a}\right)$. e a linha tracejada é a trajetória de $-|h(t)|$. Quando $t \notin \phi$, temos que, conforme Figura 5.3

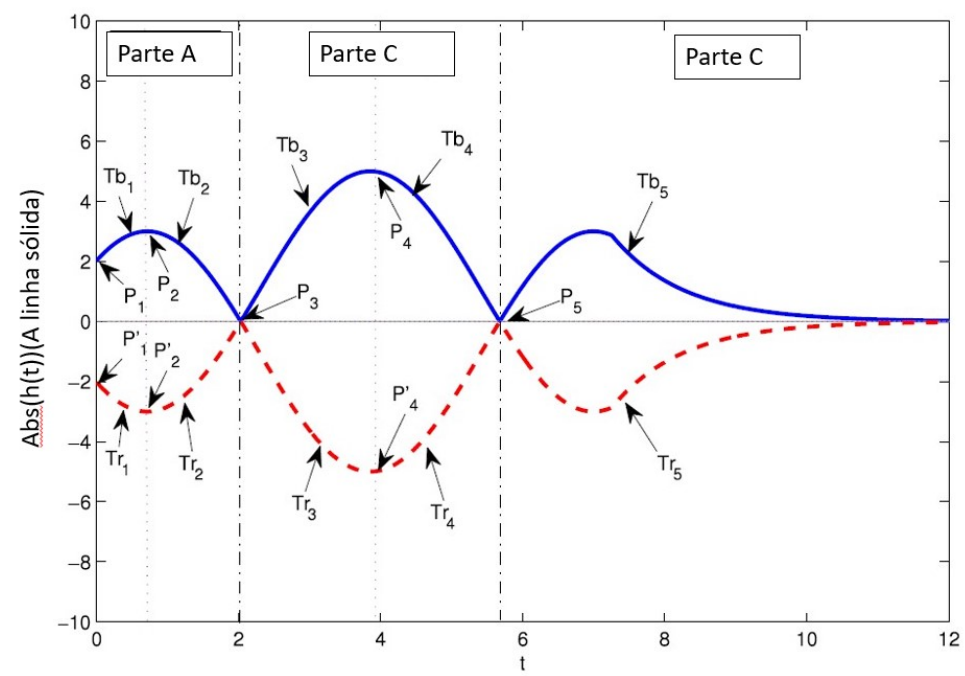

Figura 5.3: Exemplo de uma trajetória de $|h(t)|$. Fonte: [61]. 


$$
\begin{aligned}
{ }_{0}^{\mathrm{C}} \mathrm{D}_{t}^{\alpha}\left|h\left(t^{+}\right)\right| & =\frac{1}{\Gamma(1-\alpha)} \int_{0}^{t} \frac{|h(\tau)|^{\prime}}{(t-\tau)^{\alpha}} d \tau \\
& =\frac{1}{\Gamma(1-\alpha)}\left(\int_{0}^{X p 3} \frac{|h(\tau)|^{\prime}}{(t-\tau)^{\alpha}} d \tau+\int_{X p 3}^{X p 5} \frac{|h(\tau)|^{\prime}}{(t-\tau)^{\alpha}} d \tau+\int_{X p 5}^{t} \frac{|h(\tau)|^{\prime}}{(t-\tau)^{\alpha}} d \tau\right)
\end{aligned}
$$

e

$\operatorname{sgn}(h(t)){ }_{0}^{\mathrm{C}} \mathrm{D}_{t}^{\alpha} h(t)=$

$$
=\frac{1}{\Gamma(1-\alpha)}\left(\int_{0}^{X p 3} \frac{|\operatorname{sgn}(h(t)) h(\tau)|^{\prime}}{(t-\tau)^{\alpha}} d \tau+\int_{X p 3}^{X p 5} \frac{|\operatorname{sgn}(h(t)) h(\tau)|^{\prime}}{(t-\tau)^{\alpha}} d \tau+\int_{X p 5}^{t} \frac{|\operatorname{sgn}(h(t)) h(\tau)|^{\prime}}{(t-\tau)^{\alpha}} d \tau\right)
$$

Na parte A, se $\operatorname{sgn}(h(t)) h(\tau)=|h(\tau)|$, nós temos que

$$
\int_{0}^{X p_{3}} \frac{|h(\tau)|^{\prime}}{(t-\tau)^{\alpha}} d \tau=\int_{0}^{X p_{3}} \frac{(\operatorname{sgn}(h(t)) h(\tau))^{\prime}}{(t-\tau)^{\alpha}} d \tau
$$

$\mathrm{e}$

$$
\int_{0}^{X p_{3}} \frac{|h(\tau)|^{\prime}}{(t-\tau)^{\alpha}} d \tau=\int_{0}^{X p_{2}} \frac{|h(\tau)|^{\prime}}{(t-\tau)^{\alpha}} d \tau+\int_{X p_{2}}^{X p_{3}} \frac{|h(\tau)|^{\prime}}{(t-\tau)^{\alpha}} d \tau
$$

Perceba que, para $\alpha<1$ e $\tau \in\left[0, X p_{2}\right), \frac{1}{(t-\tau)^{\alpha}}<\frac{1}{\left(t-X p_{2}\right)^{\alpha}}$. De maneira análoga, se $\tau \in\left(X p_{2}, 0\right], \frac{1}{(t-\tau)^{\alpha}}>$ $\frac{1}{\left(t-X p_{2}\right)^{\alpha}}$. A primeira integral do lado direito da Equação 5.12 é positiva e $|h(\tau)|^{\prime}>0$, enquanto a segunda integral é negativa e $|h(\tau)|^{\prime}<0$. Assim,

$$
\begin{aligned}
\int_{0}^{X p_{3}} \frac{|h(\tau)|^{\prime}}{(t-\tau)^{\alpha}} d \tau & <\frac{1}{\left(t-X p_{2}\right)^{\alpha}} \int_{0}^{X p_{2}}|h(t)|^{\prime} d \tau+\frac{1}{\left(t-X p_{2}\right)^{\alpha}} \int_{X p_{2}}^{X p_{3}}|h(t)|^{\prime} d \tau \\
& =\frac{-Y p_{1}}{\left(t-X p_{2}\right)^{\alpha}} \leq 0 .
\end{aligned}
$$

Agora, se $\operatorname{sgn}(h(t)) h(\tau)=-|h(\tau)|$, teremos

$$
\int_{0}^{X p_{3}} \frac{(\operatorname{sgn}(h(t)) h(\tau))^{\prime}}{(t-\tau)^{\alpha}} d \tau=-\int_{0}^{X p_{3}} \frac{|h(\tau)|^{\prime}}{(t-\tau)^{\alpha}} d \tau>0>\int_{0}^{X p_{3}} \frac{|h(\tau)|^{\prime}}{(t-\tau)^{\alpha}} d \tau .
$$

Na parte B, se $\operatorname{sgn}(h(t)) h(\tau)=|h(\tau)|$, então

$$
\int_{X p_{3}}^{X p_{5}} \frac{|h(\tau)|^{\prime}}{(t-\tau)^{\alpha}} d \tau=\int_{X p_{3}}^{X p_{5}} \frac{(\operatorname{sgn}(h(t)) h(\tau))^{\prime}}{(t-\tau)^{\alpha}} d \tau .
$$

De forma análoga à parte $\mathrm{A}$, observando a Figura 5.3 , temos que

$$
\begin{aligned}
\int_{X p_{3}}^{X p_{5}} \frac{|h(\tau)|^{\prime}}{(t-\tau)^{\alpha}} d \tau= & \int_{X p_{3}}^{X p_{4}} \frac{|h(\tau)|^{\prime}}{(t-\tau)^{\alpha}} d \tau+\int_{X p_{4}}^{X p_{5}} \frac{|h(\tau)|^{\prime}}{(t-\tau)^{\alpha}} d \tau \\
& <\frac{1}{\left(t-X p_{4}\right)^{\alpha}} \int_{X p_{3}}^{X p_{4}}|h(t)|^{\prime} d \tau+\frac{1}{\left(t-X p_{4}\right)^{\alpha}} \int_{X p_{4}}^{X p_{5}}|h(t)|^{\prime} d \tau \\
& =\frac{Y p_{5}-Y p_{3}}{\left(t-X p_{4}\right)^{\alpha}} \leq 0 .
\end{aligned}
$$

Se $\operatorname{sgn}(h(t)) h(\tau)=-|h(\tau)|$, temos que, na parte B

$$
\int_{X p_{3}}^{X p_{5}} \frac{(\operatorname{sgn}(h(t)) h(\tau))^{\prime}}{(t-\tau)^{\alpha}} d \tau=-\int_{X p_{3}}^{X p_{5}} \frac{|h(\tau)|^{\prime}}{(t-\tau)^{\alpha}} d \tau>0>\int_{X p_{3}}^{X p_{5}} \frac{|h(\tau)|^{\prime}}{(t-\tau)^{\alpha}} d \tau .
$$


Na parte $\mathrm{C}$, se $h(t)>0$ ou $h(t)<0$ teremos

$$
\int_{X p_{5}}^{t} \frac{(\operatorname{sgn}(h(t)) h(\tau))^{\prime}}{(t-\tau)^{\alpha}} d \tau=\int_{X p_{5}}^{t} \frac{|h(\tau)|^{\prime}}{(t-\tau)^{\alpha}} d \tau .
$$

Então, para concluirmos, temos que

$$
{ }_{0}^{\mathrm{C}} \mathrm{D}_{t}^{\alpha}\left|h\left(t^{+}\right)\right| \leq \frac{1}{\Gamma(1-\alpha)} \int_{X p_{0}}^{t} \frac{(\operatorname{sgn}(h(t)) h(\tau))^{\prime}}{(t-\tau)^{\alpha}} d \tau=\operatorname{sgn}(h(t)){ }_{0}^{\mathrm{C}} \mathrm{D}_{t}^{\alpha} h(t)
$$

para qualquer $t \notin \phi$. Como a medida de $\phi$ e zero, a desigualdade (5.11) é quase sempre assegurada. Com mais do que um ponto extremo na parte B, a desigualdade (5.11) ainda seria assegurada. Na Figura 5.2 , dividimos a parte B de $|h(t)|$ em 10 partes por pontos. Chegaríamos em resultados similares se fizéssemos a integração em $\left[T_{1}, T_{10}\right],\left[T_{2}, t_{3}\right],\left[T_{4}, t_{9}\right],\left[T_{5}, t_{6}\right],\left[T_{7}, T_{8}\right]$ respectivamente.

Podemos ver que o Teorema (5.3) estabelece uma conexão entre ${ }^{C} \mathrm{D}_{t}^{\alpha} \mid h\left(t^{+} \mid\right.$e ${ }^{\mathrm{C}} \mathrm{D}_{t}^{\alpha} h(t)$, o que é necessário para verificar a 1-norma de $x$ satisfazendo a desigualdade (5.10).

Considere a RNHF da Equação 4.15

$$
{ }_{0}^{\mathrm{C}} D_{t}^{\alpha} v_{k}(t)=-a_{k} v_{k}(t)+\sum_{k=1}^{n} b_{k j} \varphi_{j}\left(v_{j}(t)\right)+I_{k}(t)
$$

onde $a_{k}=\frac{1}{R_{k} F_{k}}, b_{k j}=\frac{w_{k j}}{F_{k}}$ e $I_{k}$ denota a corrente aplicada exteriormente que pode ou não depender do tempo. Caso $I_{k}$ não dependa do tempo, o seguinte teorema é valido:

Teorema 5.4 (Estabilidade de Mittag-Leffler para RNHF com entradas externas contantes) [61] Considere as seguintes suposições a respeito do sistema (5.14):

(A1) As funções de ativação são contínuas e Lipischitizianas em $\mathbb{R}$ com constante de Lipschitz $L_{j}>0$, ou seja

$$
\left|f_{j}\left(v_{1}\right)-f_{j}\left(v_{2}\right)\right| \leq L_{j}\left|v_{1}-v_{2}\right| .
$$

que assegura a existência e unicidade das soluções.

(A2) Existe constantes positivas $c_{k}(k=1, \ldots, n)$ tal que

$$
c_{k}=a_{k}-\sum_{j=1}^{n}\left|b_{j k}\right| L_{k}>0 .
$$

Sob estas condições, o sistema 5.14 é globalmente Mittag-Leffler estável.

Demonstração. Primeiramente, vamos provar a existência e unicidade do ponto de equilíbrio do sistema 5.14 Considere a aplicação $H(p)=\left(H\left(p_{1}\right), \ldots, H\left(p_{n}\right)\right)^{T}$, onde

$$
H_{k}(p)=\sum_{j=1}^{n} b_{k j} \varphi_{k}\left(\frac{p_{j}}{a_{j}}\right)+I_{k}, i=1, \ldots, n,
$$

para $p=\left(p_{1}, \ldots, p_{n}\right)^{T} \in \mathbb{R}^{n}$. Usando a suposição $(A 1)$ para dois vetores quaisquer $p, q \in \mathbb{R}^{n}$, temos

$$
\left|H_{i}(p)-H_{i}(q)\right|=\left|\sum_{j=1}^{n} b_{k j}\left(\varphi_{j}\left(\frac{p_{j}}{a_{j}}\right)-\varphi_{j}\left(\frac{q_{j}}{a_{j}}\right)\right)\right| \leq \sum_{j=1}^{n} \frac{\left|b_{k j}\right| I_{j}}{a_{j}}\left|p_{j}-q_{j}\right| .
$$


Seja a 1-norma de $x,\|x\|_{1}=\sum_{k=1}^{n}\left|x_{k}\right|$, para $v=\left(x_{1}, \ldots, x_{n}\right)^{T} \in \mathbb{R}^{n}$. Assim,

$$
\begin{aligned}
\|H(p)-H(q)\|_{1}=\sum_{k}^{n}\left|H_{k}(p)-H_{i}(q)\right| & \leq \sum_{k}^{n} \sum_{j=1}^{n} \frac{\left|b_{k j}\right| I_{j}}{a_{j}}\left|p_{j}-q_{j}\right| \\
& =\sum_{k}^{n}\left(\sum_{j=1}^{n} \frac{\left|b_{k j}\right| I_{j}}{a_{j}}\right)\left|p_{j}-q_{j}\right| .
\end{aligned}
$$

Da suposição $(A 2)$ temos que $\sum_{j=1}^{n}\left|b_{j k}\right| I_{k}<a_{k} \Rightarrow \sum_{j=1}^{n} \frac{\left|b_{j k}\right| I_{k}}{a_{k}}<1$. Então,

$$
\sum_{k}^{n}\left(\sum_{j=1}^{n} \frac{\left|b_{k j}\right| I_{j}}{a_{j}}\right)\left|p_{j}-q_{j}\right|<\sum_{k}^{n}\left|p_{j}-q_{j}\right|=|| p-q \|_{1} .
$$

Da Equação (5.17), podemos ver que a aplicação $H: \mathbb{R}^{n} \rightarrow \mathbb{R}^{n}$ é uma contração em $\mathbb{R}^{n}$, pois $\|H(p)-H(q)\|_{1}<\|p-q\|_{1}$. Assim, pelo teorema do ponto fixo de Banach, existe um único ponto fixo $\bar{p} \in \mathbb{R}^{n}$ tal que $H(\bar{p})=\bar{p}$, ou seja,

$$
\bar{p}_{k}=\sum_{j=1}^{n} b_{j k} \varphi_{j}\left(\frac{\bar{p}}{a_{j}}\right)+I_{k}, i=1, \ldots, n .
$$

Se denotarmos $\bar{v}_{k}=\frac{\bar{p}_{k}}{a_{k}}$ para $i=1, \ldots, n$, então substituindo na Equação 5.18 , temos

$$
-a_{k} \bar{v}_{k}+\sum_{j=1}^{n} b_{j k} \varphi_{j}\left(v_{j}\right)+I_{k}=0, i=1, \ldots, n,
$$

o que mostra que $v=\left(v_{-} 1, \ldots, v_{n}\right)^{T}$ é o único ponto de equilíbrio do sistema 5.14. Vamos provar agora que o ponto de equilíbrio desse sistema é Mittag-Leffler estável (de acordo com o Teorema 5.2. Sejam duas soluções $x(t)$ e $y(t)$ do sistema (5.14) com condições iniciais diferentes e $e(t)=$ $\left(e_{1}(t), \ldots, e_{n}(t)\right)^{T}=y(t)-x(t)$. Neste caso, $e(t)$ também seria solução, ou seja,

$$
{ }_{0}^{\mathrm{C}} \mathrm{D}_{t}^{\alpha} e_{k}(t)=-a_{k} e_{k}(t)+\sum_{j=1}^{n} b_{k j}\left(\varphi_{j}\left(y_{j}(t)\right)-\varphi_{j}\left(x_{j}(t)\right)\right)
$$

Para $\alpha<1, e_{k}(t)$ deve ser continuamente diferenciável. Então $\frac{d|e(t)|}{d t}$ é contínua por partes (não é diferenciável apenas onde $\left.e(t)=0 \operatorname{com} e^{\prime}(t) \neq 0\right)$, e $\lim _{\tau \rightarrow t^{+}} \frac{d|e(\tau)|}{d \tau}$ existe pata $t \in[0, \infty)$. Então construímos uma função de Lyapunov como

$$
V(t, e(t))=\|e(t)\|_{1}=\sum_{k=1}^{n}\left|e_{k}(t)\right| .
$$

Veja que claramente a função 5.20 satisfaz a condição (5.9). De acordo com o Teorema 5.3

$$
\begin{aligned}
{ }_{0}^{\mathrm{C}} \mathrm{D}_{t}^{\alpha} V\left(t^{+}, e\left(t^{+}\right)\right) & =\sum_{k-1}^{n}{ }_{0}^{\mathrm{C}} \mathrm{D}_{t}^{\alpha}\left|e_{k}\left(t^{+}\right)\right| \leq \sum_{k=1}^{n} \operatorname{sgn}\left(e_{k}(t)\right){ }_{0}^{\mathrm{C}} \mathrm{D}_{t}^{\alpha} e_{k}(t) \\
& =\sum_{k=1}^{n} \operatorname{sgn}\left(e_{k}(t)\right)\left[-a_{k} e_{k}(t)+\sum_{j=1}^{n} b_{k j}\left(\varphi_{j}\left(y_{j}(t)\right)-\varphi_{j}\left(x_{j}(t)\right)\right)\right] .
\end{aligned}
$$


Da suposição (A1) temos que, continuando,

$$
\begin{aligned}
{ }_{0}^{\mathrm{C}} \mathrm{D}_{t}^{\alpha} V\left(t^{+}, e\left(t^{+}\right)\right) & \leq \sum_{k=1}^{n}\left[-a_{k} e_{k}(t)+\sum_{j=1}^{n} L_{j}\left|b_{k j}\right|\left|e_{j}(t)\right|\right] \\
& =\sum_{k=1}^{n}\left[-a_{k} e_{k}(t)+\sum_{j=1}^{n} L_{k}\left|b_{j k}\right|\left|e_{k}(t)\right|\right] \\
& =\sum_{k=1}^{n}\left[a_{k}-\sum_{j=1}^{n} L_{k}\left|b_{j k}\right|\right]\left|e_{k}(t)\right|,
\end{aligned}
$$

e da suposição $(A 2)$ chegamos a

$$
{ }_{0}^{\mathrm{C}} \mathrm{D}_{t}^{\alpha} V\left(t^{+}, e\left(t^{+}\right)\right) \leq-c\|e(t)\|_{1},
$$

onde $c=\min \left\{c_{1}, \ldots, c_{n}\right\}$.

Assim, a desigualdade (5.21) satisfaz a condição 5.10. Portanto, a solução do sistema (5.19) é globalmente Mittag-Leffler, ou seja,

$$
\|e(t)\|_{1} \leq V(0, e(0)) E_{\alpha}\left(-c t^{\alpha}\right) .
$$

Sendo $\bar{v}$ o único ponto de equilíbrio e $v(t)$ uma solução do sistema (5.14), então

$$
\|v(t)-\bar{v}\|_{1} \leq V(0, v(0)-\bar{v}) E_{\alpha}\left(-c t^{\alpha}\right),
$$

o que mostra que o sistema (5.14) é globalmente Mittag-Leffler estável.

No caso em que o sistema (5.14) tenha $I_{k}$ dependente do tempo, tal sistema torna-se não autônomo e talvez não tenha nenhum ponto de equilíbrio. Apesar disto, sua estabilidade ainda pode ser assegurada. Não apenas isto, mas podemos assegurar que tal sistema tem um ponto de equilíbrio uniformemente estável e estabelecer um raio $\delta$ onde a trajetória $x(t)$ se mantenha em seu interior.

Teorema 5.5 (Estabilidade de Mittag-Leffler para RNHF com entradas externas dinâmicas) [61] Suponha que as condições (A1) e (A2) do Teorema 5.4 são satisfeitas para o sistema (5.14) com $I_{k}$ limitado por $M_{k}$ e dependente do tempo. Neste caso, o sistema é uniformemente estável e existe $T \geq t_{0}$ tal que para todo $t \geq T$ e qualquer solução $v(t)$,

$$
\|v(t)\| \leq \frac{W}{c}+\epsilon
$$

onde $c=\min \left\{c_{1}, \ldots, c_{n}\right\}, W=\sum_{k=1}^{n} \sum_{j=1}^{n}\left(\left|b_{k j}\right|\left|\varphi_{k}(0)\right|+M_{k}\right)$ e $0<\epsilon<<1$.

Demonstração. Podemos construir uma função de Lyapunov $V(t, x(t))=\|x(t)\|_{1}$. Procedendo de forma análoga à demonstração do Teorema 5.4, temos que

$$
\begin{aligned}
{ }_{0}^{\mathrm{C}} \mathrm{D}_{t}^{\alpha} V\left(t^{+} x\left(t^{+}\right)\right. & =\sum_{i=1}^{n}{ }_{0}^{\mathrm{C}} \mathrm{D}_{t}^{\alpha}\left|x_{i}\left(t^{+}\right)\right| \leq \sum_{i=1}^{n} \operatorname{sgn}\left(x_{i}(t)\right){ }_{0}^{\mathrm{C}} \mathrm{D}_{t}^{\alpha} x_{i}(t) \\
& =\sum_{i=1}^{n} \operatorname{sgn}\left(x_{i}(t)\right)\left[-a_{i} x_{i}(t)+\sum_{j=1}^{n} b_{i j}\left(f_{j}\left(x_{j}(t)\right)-f_{j}(0)\right)+\sum_{j=1}^{n} b_{i j} f_{j}(0)+I_{t}\right] \\
& \leq-\sum_{i=1}^{n}\left[a_{i}-\sum_{j=1}^{n} I_{i}\left|b_{j i}\right|\right]\left|x_{t}\right|+\sum_{i=1}^{n}\left(\sum_{j=1}^{n}\left|b_{j i}\right|\left|f_{i}(0)\right|+M_{i}\right) \\
& \leq-c V(t, x(t))+W=-c V\left(t^{+}, c\left(t^{+}\right)\right)+W
\end{aligned}
$$


Vamos considerar o sistema com uma dimensão ${ }_{0}^{\mathrm{C}} \mathrm{D}_{t}^{\alpha} z(t)=-c z(t)+W$. De acordo com o Teorema 5.4. o único ponto de equilíbrio desse sistema, $\bar{z}=\frac{W}{c}$ é Mittag-Leffler estável, e consequentemente assintoticamente estável, ou seja, $\lim _{t \rightarrow \infty}\|z(t)-\bar{z}\|=0$. Devido ao Lema 3.9, como ${ }_{0}^{\mathrm{C}} \mathrm{D}_{t}^{\alpha} V(t, x(t)) \leq$ ${ }_{0}^{\mathrm{C}} \mathrm{D}_{t}^{\alpha} z(t)$, temos que $V(t, x(t)) \leq z(t) \operatorname{com} V(0, x(0))=z(0)$. Então, existe $T \geq t_{0}$ tal que, para todo $t \geq T$,

$$
\|x(t)\| \leq \lim _{t \rightarrow+\infty} z(t)+\epsilon=\frac{W}{c}+\epsilon .
$$

Exemplo 5.1 Vamos considerar agora a rede de Hopfield do Exemplo (5.1) com a diferença da derivada ser fracionária,

$$
\begin{aligned}
& { }_{0}^{\mathrm{C}} D_{t}^{\alpha} v_{1}(t)=-6 v_{1}(t)+2 \operatorname{sen}\left(v_{1}(t)\right)+\operatorname{sen}\left(v_{2}(t)\right)-3 \operatorname{sen}\left(v_{3}(t)\right)+I_{1} \\
& { }_{0}^{\mathrm{C}} D_{t}^{\alpha} v_{2}(t)=-5 v_{2}(t)-2 \operatorname{sen}\left(v_{1}(t)\right)-0.4 \operatorname{sen}\left(v_{2}(t)\right)+\operatorname{sen}\left(v_{3}(t)\right)+I_{2} \\
& { }_{0}^{\mathrm{C}} D_{t}^{\alpha} v_{3}(t)=-8 v_{3}(t)+\operatorname{sen}\left(v_{1}(t)-2.5 \operatorname{sen}\left(v_{2}(t)\right)+3.5 \operatorname{sen}\left(v_{3}(t)\right)+I_{3}\right.
\end{aligned}
$$

Supondo as entradas $I_{1}=\pi-4, I_{2}=2$ e $I_{3}=3-4 \pi$, ou seja, entradas constantes, as condições (A1) e (A2) do Teorema 5.4 são verificadas facilmente. Então, o único ponto de equilíbrio desse sistema é globalmente Mittag-Leffler estável, como pode ser verificado observando as Figura 5.4 para diferentes valores de $\alpha$ e com condição inicial $(4,4,4)^{T}$.

Agora, suponha entradas externas dependentes do tempo, $I_{1}(t)=0.8 \cos (t), I_{2}(t)=-0.2 \operatorname{sen}(t)$ e $I_{3}(t)=\frac{t-1}{t+1}$. As condições (A1) e (A2) ainda são asseguradas e, de acordo com o Teorema 5.5 o sistema é uniformemente estável. Então existe um tempo $T>t_{0}$ tal que $\left|v_{k}(t)\right| \leq\|x(t)\| \leq \frac{W}{C}+\epsilon$. Como $\left|I_{k}\right| \leq M_{k}$, podemos escolher $M_{1}=0.8, M_{2}=0.2$ e $M_{3}=1 \mathrm{e}$

$$
\begin{aligned}
W & =\sum_{k=1}^{3} \sum_{j=1}^{3}\left(\left|b_{i j}\right||\operatorname{sen}(0)|+M_{k}\right)=2 \\
c_{1} & =6-\sum_{j=1}^{3}\left|b_{j 1}\right|=5 \\
c_{2} & =5-\sum_{j=1}^{3}\left|b_{j 2}\right|=3.1 \\
c_{3} & =8-\sum_{j=1}^{3}\left|b_{j 3}\right|=6.5,
\end{aligned}
$$

o que leva a $\left|v_{k}(t)\right| \leq 0.65$ supondo $\epsilon=0.01$. Com diferentes valores para $\alpha$ e com condição inicial $(4,4,4)^{T}$, a Figura 5.5 mostra que, para $T>5$ por exemplo, todas as soluções satisfazem $\left|v_{k}\right| \leq 0.5, k=1,2,3$. 

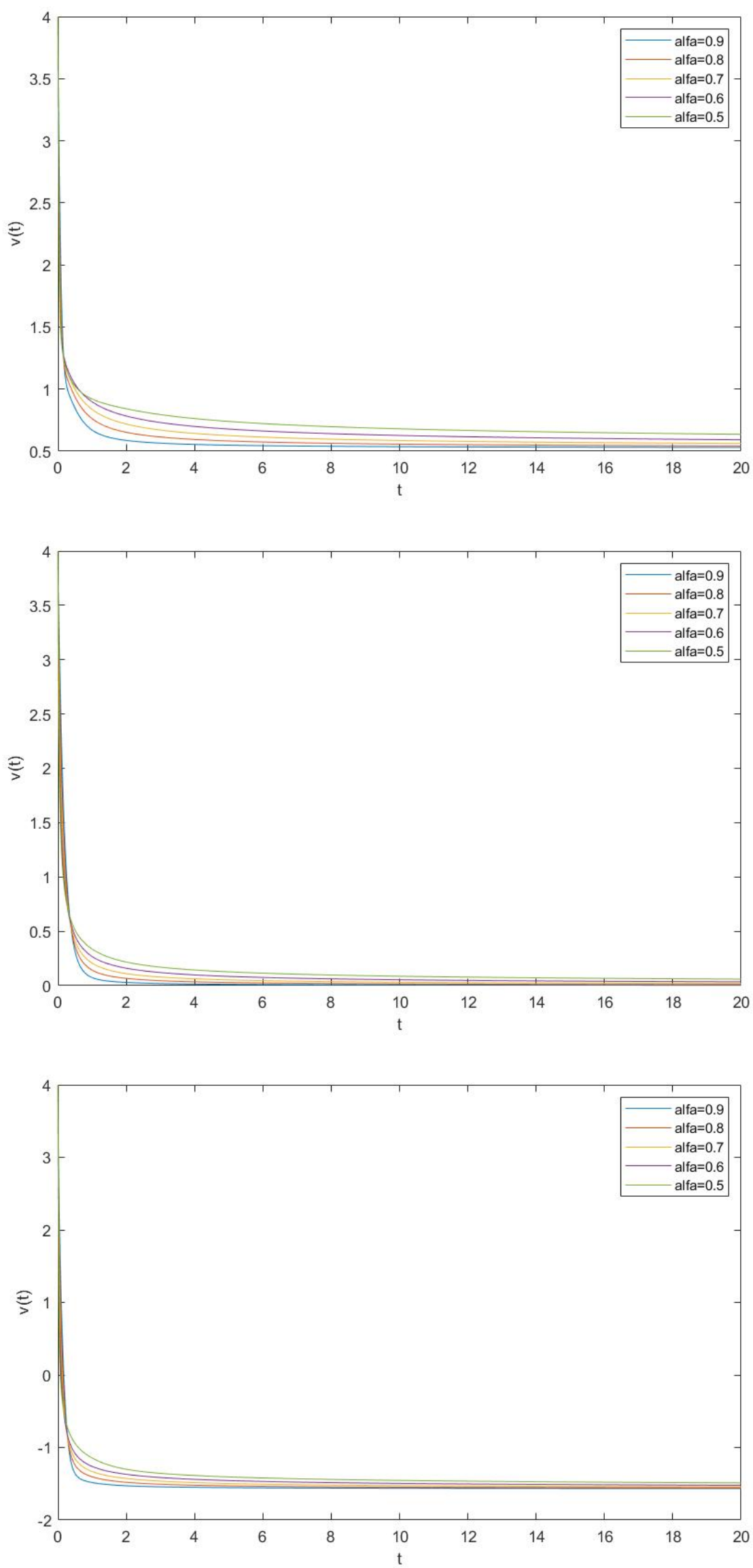

Figura 5.4: Solução da FHNN 5.5 com condiçấo inicial $(4,4,4)^{T}$ para os valores de $\alpha=0.9,0.8,0.7,0.6$ e 0.5 em cada uma das coordenadas de $v(t)=\left[v_{1}(t), v_{2}(r), v_{3}(t)\right]^{T}$. 

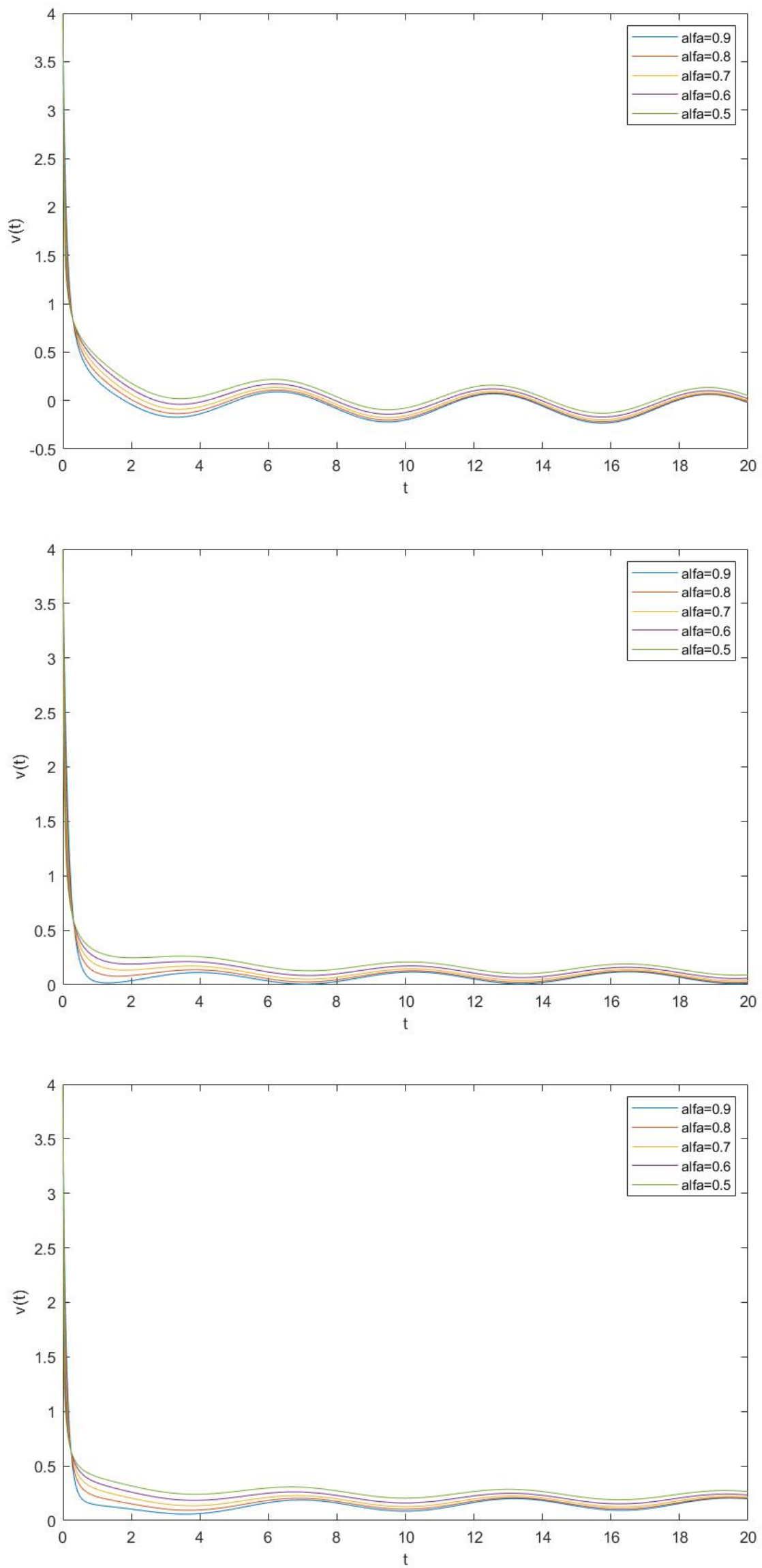

Figura 5.5: Solução da FHNN com condição inicial $(4,4,4)^{T}$ e constantes externas dependentes do tempo para os valores de $\alpha=0.9,0.8,0.7,0.6$ e 0.5 em cada uma das coordenadas de $v(t)=\left[v_{1}(t), v_{2}(r), v_{3}(t)\right]^{T}$. 


\section{Capítulo 6}

\section{Considerações Finais}

Neste trabalho, apresentamos inicialmente as principais propriedades das derivadas e integrais fracionárias com a interpretação de Caputo e Riemann-Liouville. Expusemos uma interpretação física para essas derivadas, baseada no conceito de memória de um sistema, e estudamos ferramentas necessárias para o estudo das equações diferenciais ordinárias fracionárias, como a função de Mittag-Leffler e a transformada de Laplace. Com isso, como uma primeira proposta deste trabalho, demonstramos os teoremas de existência e unicidade de uma EDOF, utilizando os clássicos teoremas de ponto fixo da análise funcional.

O estudo da estabilidade de pontos de equilíbrio de equações diferenciais fracionárias é um área bastante investigada atualmente e possui diversas aplicações. Neste trabalho mostramos um critério de estabilidade proposto em 2009, a estabilidade de Mittag-Leffler, que tem a estabilidade em lei de potência e exponencial como casos particulares. A partir disto, deduziu-se uma extensão do método direto de Lyapunov para sistemas de ordens não inteiras.

Para demonstrar a efetividade da estabilidade de Mittag-Leffler, estudamos sua aplicação para os pontos de equilíbrio de redes neurais Hopfield de ordem fracionária. Uma condição suficiente de estabilidade de Mittag-Leffler para redes neurais Hopfield de ordem fracionária com entrada constante é dada. A estabilidade uniforme de redes neurais Hopfield de ordem fracionária com entrada dependente do tempo foi analisada e discutida.

Apresentamos também uma generalização do capacitor, onde o capacitor comum da rede neural Hopfield de ordem inteira de tempo contínuo é substituído pelo capacitor fracionário, dando origem ao chamado modelo de rede neural de Hopfield de ordem fracionária.

Sobre a rede de Hopfield fracionária, ainda é um tópico de pesquisa muito recente e promissor com relação à sua dinâmica e comportamento dos pontos de equilíbrio e, devido ao fato de modelos de ordem fracionária possuírem melhor descrição de memória, possíveis aplicações futuras podem demonstrar sua efetividade em comparação às redes de Hopfield em ordem inteira.

Sobre sugestões de pesquisas futuras, uma extensão natural deste trabalho seria estudar critérios de estabilidade de redes neurais fracionárias com mais de um ponto de equilíbrio e abordar redes neurais de Hopfield fracionárias atrasadas e impulsivas, já que tais redes podem sofrer perturbações impulsivas que podem afetar comportamentos dinâmicos dos sistemas, assim como atrasos de tempo que é inevitável na prática e capaz de causar oscilações ou instabilidades em sistemas dinâmicos. 


\section{Apêndice A}

\section{Teoremas de Ponto Fixo}

Vamos primeiro relembrar alguns resultados úteis em análise funcional.

Definição A.1 (Espaço Métrico) Seja U um conjunto não vazio. Uma métrica sobre um conjunto $U$ é uma aplicação $d: U \times U \rightarrow \Re$ que a cada par de elementos $u, v \in U$ associa um número real positivo $d(u, v)$, que será denominado como distância entre u e $v$, de modo que, para qualquer elemento $u, v, w \in U$ tem-se:

1. $d(u, v) \geq 0$ e d $(u, v)=0 \Leftrightarrow u=v$;

2. $d(u, v)=d(v, u)$ (simetria)

3. $d(u, w) \leq d(u, v)+d(v, z)$ (desigualdade triangular)

O par $(U, d)$ é denominado espaço métrico formado por um conjunto não vazio $U$ e uma métrica dem $U$.

Definição A.2 (Espaço métrico completo) Seja $(U, d)$ um espaço métrico. Este espaço métrico é completo quando toda sequência de Cauchy em $U$ é convergente em $U$.

Definição A.3 (Equicontinuidade) Uma família de funções $F \in C[a, b]$ é dita ser equicontínua quando, para cada $\epsilon>0$ existe algum $\delta>0$ tal que para $f \in F$ e $x, x^{*} \in[a, b]$ com $\left|x-x^{*}\right| \leq \delta$, nós temos $\left|f(x)-f\left(x^{*}\right)\right|<\epsilon$.

Definição A.4 (Conjunto Relativamente Compacto) Seja $(U, d)$ um espaço métrico e $F \subseteq$ $U$. $O$ conjunto $F$ é relativamente compacto em $U$ se o fecho de $F$ é um subconjunto compacto de E.

O teorema a seguir é um resultado muito importante na Análise, pois determina quais são as condições necessárias e suficientes para que um conjunto de funções, com valores em um espaço métrico $(U, d)$, seja relativamente compacto em $U$.

Teorema A.1 (Teorema de Arzelà-Ascoli) Seja $F \in C[a, b]$ para algum $a<b$ e admita que este conjunto está equipado com a norma do supremo. Então, $F$ é relativamente compacto em $C[a, b]$ se $F$ é equicontínuo e uniformemente limitado (ou seja, existe uma constante $C>0$ tal que $\|f\| \leq C$ para cada $f \in F$ ).

Demonstração: Uma demonstração deste teorema pode ser encontrada em [5].

Para demonstrar os teoremas de existência e unicidade em equações diferencias ordinárias fracionárias, usamos os teoremas de ponto fixo de Weissinger e Schauder. O primeiro é uma generalização do ponto fixo de Banach e pode ser enunciado como: 
Teorema A.2 (Teorema do Ponto Fixo de Weissinger) Seja $(U, d) m$ espaço métrico nãovazio e completo, e $\alpha_{j} \geq 0$ para cada $j \in \mathcal{N}$ e tal que $\sum_{j=0}^{\infty} \alpha_{j}$ converge. Além disso, seja a aplicação $A: U \rightarrow U$ satisfazendo a desigualdade

$$
d\left(A^{j} u, A^{j} v\right) \leq \alpha_{j} d(u, v)
$$

para cada $j \in \mathcal{N}$ e cada $u, v \in U$. Então A tem um único ponto fixo determinado $u^{*}$ e, para qualquer $u_{0} \in U$, a sequência $\left(A^{j} u_{0}\right)_{j=1}^{\infty}$ converge para o ponto fixo $u^{*}$.

Demonstração: Pode ser encontrada em [57].

Uma consequência imediata deste teorema é o teorema a seguir

Teorema A.3 (Teorema do Ponto Fixo de Bannach) Seja $(U, d)$ um espaço métrico completo não vazio. Seja $0 \leq \alpha<1$ e a aplicação $A: U \rightarrow U$ satisfazendo a desigualdade

$$
d(A u, A v) \leq \alpha d(u, v)
$$

para todo $u, v \in U$. Então A tem um único ponto fixo $u^{*}$. Além disso, para qualquer $u_{0} \in U$, a sequência $\left(A^{j} u\right)_{j=1}^{\infty}$ converge para o ponto fixo $u^{*}$.

Quando queremos assegurar apenas a existência de ponto fixo, podemos trabalhar com uma hipótese mais fraca para o operador em questão. O teorema do ponto de Schauder assegura as hipóteses para a existência de soluções:

Teorema A.4 (Teorema do Ponto Fixo de Schauder) Seja (E,d) um espaço métrico completo, $U$ um conjunto convexo e fechado de $E$, e a aplicação $A: U \rightarrow U$ tal que o conjunto $\{A u: u \in U\}$ é relativamente compacto em E. Então A tem pelo menos um ponto fixo.

Demonstração: Pode ser encontrada em [30]. 


\section{Apêndice B}

\section{Aplicação no Matlab}

A solução do sistema de equações diferenciais ordinárias dada pela rede neural do exemplo 5.1 foi encontrada com o uso do algoritmo FDE12, disponível em [15]. Trata-se de uma implementação de um método numérico para resolução de EDOF's, o método preditor-corretor de Adams-BashforthMoulton, descrito em [13].

Algoritmo usado para o Exemplo 5.1

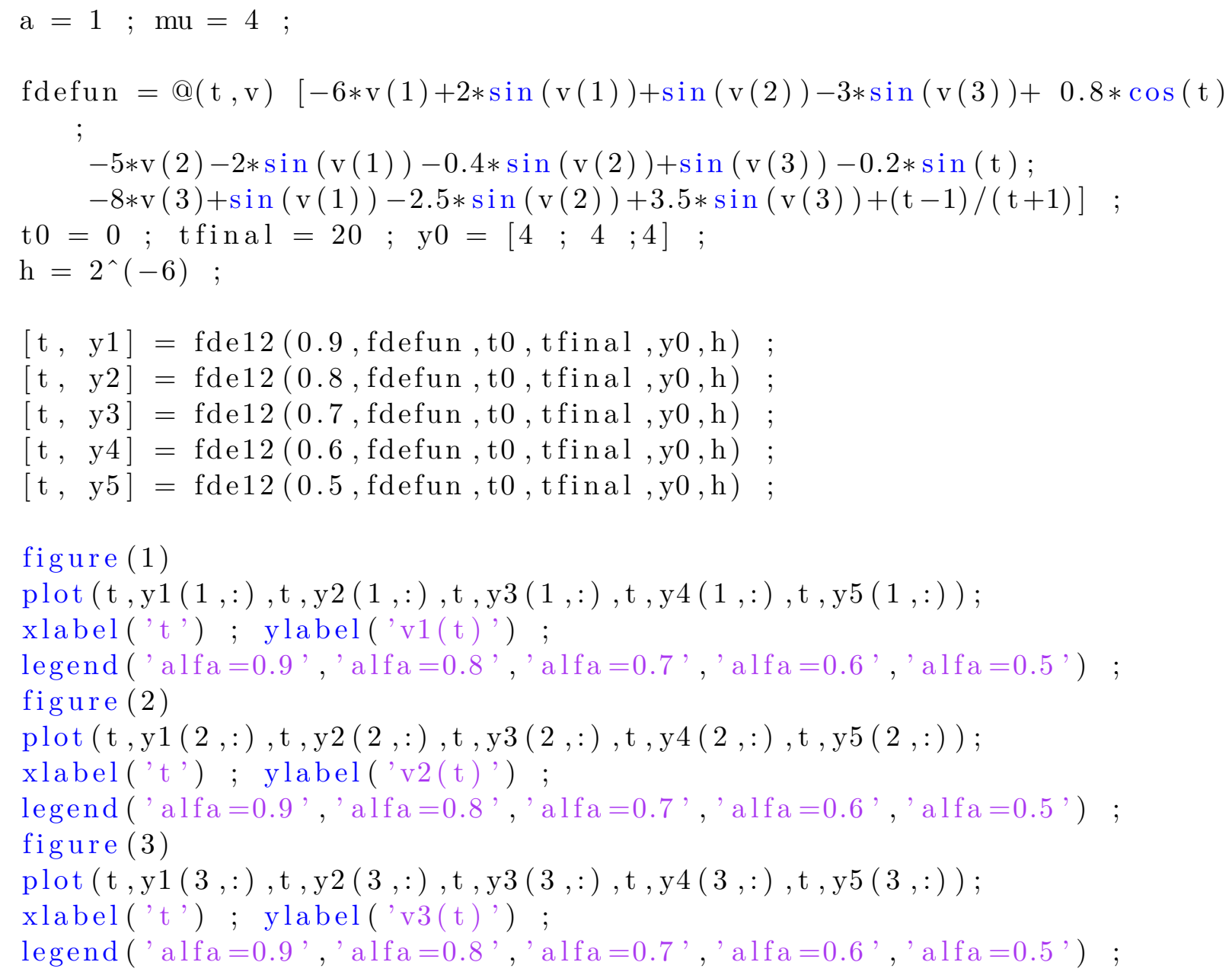




\section{Referências Bibliográficas}

[1] George E Andrews, Richard Askey, and Ranjan Roy. Special Functions, volume 71. Cambridge university press, 2000.

[2] Bijnan Bandyopadhyay and Shyam Kamal. Solution, stability and realization of fractional order differential equation. In Stabilization and Control of Fractional Order Systems: A Sliding Mode Approach, pages 55-90. Springer, 2015.

[3] Gary W Bohannan. Analog fractional order controller in temperature and motor control applications. Journal of Vibration and Control, 14(9-10):1487-1498, 2008.

[4] Arefeh Boroomand and Mohammad B Menhaj. Fractional-order Hopfield neural networks. In International Conference on Neural Information Processing, pages 883-890, 2008.

[5] Geraldo Botelho, Daniel Pellegrino, and Eduardo Teixeira. Fundamentos de análise funcional. SBM, 2012.

[6] Eugene Butkov. Física Matemática. Livros Técnicos e Científicos, Rio de Janeiro, 1988.

[7] Michele Caputo. Linear models of dissipation whose q is almost frequency independent - ii. Geophysical Journal International, 13(5):529-539, 1967.

[8] Michele Caputo. Elasticita e dissipazione. Zanichelli, Bologna, 1969.

[9] S Reynold Chu, Rahmat Shoureshi, and Manoel Tenorio. Neural networks for system identification. IEEE Control systems magazine, 10(3):31-35, 1990.

[10] Michael A Cohen and Stephen Grossberg. Absolute stability of global pattern formation and parallel memory storage by competitive neural networks. IEEE transactions on systems, man, and cybernetics, (5):815-826, 1983.

[11] Rubens de Figueiredo Camargo. Cálculo Fracionário e Aplicações. PhD thesis, Unicamp, Campinas, 2009.

[12] Kai Diethelm. The Analysis of Fractional Differential Equations: An application-oriented exposition using differential operators of Caputo type. Springer Science \& Business Media, 2010 .

[13] Kai Diethelm and Alan D Freed. The Fracpece subroutine for the numerical solution of differential equations of fractional order. Forschung und wissenschaftliches Rechnen, 1999:5771, 1998.

[14] Manuel A Duarte-Mermoud, Norelys Aguila-Camacho, Javier A Gallegos, and Rafael CastroLinares. Using general quadratic Lyapunov functions to prove Lyapunov uniform stability for fractional order systems. Communications in Nonlinear Science and Numerical Simulation, 22(1-3):650-659, 2015. 
[15] Roberto Garrapa. Predictor-corrector PECE method for fractional differential equations. https://www.mathworks.com/matlabcentral/fileexchange/ 32918-predictor-corrector-pece-method-for-fractional-differential-equations. Accessed: 2019-05-30.

[16] Rudolf Gorenflo and Francesco Mainardi. Essentials of fractional calculus. CiteseerX, 2000.

[17] Zhi-Hong Guan, Guanrong Chen, and Yi Qin. On equilibria, stability, and instability of Hopfield neural networks. IEEE Transactions on Neural Networks, 11(2):534-540, 2000.

[18] Allon Guez, Vladimir Protopopsecu, and Jacob Barhen. On the stability, storage capacity, and design of nonlinear continuous neural networks. IEEE Transactions on Systems, Man, and Cybernetics, 18(1):80-87, 1988.

[19] Madan Gupta, Liang Jin, and Noriyasu Homma. Static and dynamic neural networks: from fundamentals to advanced theory. John Wiley \& Sons, Nova Jersey, 2004.

[20] Simon Haykin. Neural Networks and Learning Machines, volume 3. Pearson Upper Saddle River, 2009.

[21] Nicole Heymans. Dynamic measurements in long-memory materials: fractional calculus evaluation of approach to steady state. Journal of Vibration and Control, 14(9-10):1587-1596, 2008 .

[22] Alan L Hodgkin and Andrew F Huxley. A quantitative description of membrane current and its application to conduction and excitation in nerve. The Journal of physiology, 117(4):500-544, 1952.

[23] John J Hopfield and David W Tank. "Neural" computation of decisions in optimization problems. Biological cybernetics, 52(3):141-152, 1985.

[24] Jinrong $\mathrm{Hu}$, Jiliu Zhou, Yifei Pu, Yan Liu, and Yi Zhang. A novel fraction-based hopfield neural networks. In Soft Computing in Information Communication Technology, pages 143150. Springer, 2012.

[25] P. Humbert and R.P.Agarwal. Sur la fonction de Mittag-Leffler et quelques unes de ses generalizations. Bull. Sci. Math.,(Ser.II), 45:180-185, 1953.

[26] Raluca Jessop. Stability and Hopf Bifurcation Analysis of Hopfield Neural Networks with a General Distribution of Delays. PhD thesis, University of Waterloo, Waterloo, 2011.

[27] Douglas G Kelly. Stability in contractive nonlinear neural networks. IEEE Transactions on Biomedical Engineering, 37(3):231-242, 1990.

[28] Hassan K Khalil and JW Grizzle. Nonlinear systems, volume 3. Prentice Hall Upper Saddle River, NJ, 2002.

[29] AA Kilbas and JJ Trujillo. Differential equations of fractional order: methods results and problem-i. Applicable Analysis, 78(1-2):153-192, 2001.

[30] William A Kirk and Brailey Sims. Handbook of Metric Fixed Point Theory. Springer Science \& Business Media, Berlim, 2013.

[31] JP LaSalle. The extent of asymptotic stability. Proceedings of the National Academy of Sciences of the United States of America, 46(3):363, 1960.

[32] Yan Li, YangQuan Chen, and Igor Podlubny. Mittag-leffler stability of fractional order nonlinear dynamic systems. Automatica, 45(8):1965-1969, 2009. 
[33] Yan Li, YangQuan Chen, and Igor Podlubny. Stability of fractional-order nonlinear dynamic systems: Lyapunov direct method and generalized Mittag-Leffler stability. Computers $\&$ Mathematics with Applications, 59(5):1810-1821, 2010.

[34] Xiaoxin Liao, LQ Wang, and Pei Yu. Stability of Dynamical Systems, volume 5. Elsevier, Amsterdã, 2007.

[35] George F Luger and William A Stubblefield. Artificial Intelligence and the Design of Expert Systems. Benjamin-Cummings Publishing Co., Inc., San Francisco, 1990.

[36] Brian N Lundstrom, Matthew H Higgs, William J Spain, and Adrienne L Fairhall. Fractional differentiation by neocortical pyramidal neurons. Nature Neuroscience, 11(11):1335, 2008.

[37] Aleksandr Mikhailovich Lyapunov. The general problem of the stability of motion. International journal of control, 55(3):531-534, 1992.

[38] RL Magin and M Ovadia. Modeling the cardiac tissue electrode interface using fractional calculus. Journal of Vibration and Control, 14(9-10):1431-1442, 2008.

[39] Mohammad Amirian Matlob and Yousef Jamali. The concepts and applications of fractional order differential calculus in modelling of viscoelastic systems: A primer. arXiv preprint arXiv:1706.06446, 2017.

[40] K Matsuoka. On absolute stability of neural networks. Transactions of the Institute of Electrical Communication Engineering, Japan, 74:536-542, 1991.

[41] Warren S McCulloch and Walter Pitts. A logical calculus of the ideas immanent in nervous activity. The bulletin of mathematical biophysics, 5(4):115-133, 1943.

[42] Robert J McEliece, Edward C Posner, Eugene R Rodemich, and Santosh S Venkatesh. The capacity of the Hopfield associative memory. IEEE Transactions on Information Theory, $33(4): 461-482,1987$.

[43] Kenneth S Miller and Bertram Ross. An introduction to the fractional calculus and fractional differential equations. Wiley-Interscience, 1993.

[44] Masahiro Nakagawa and Kazuyuki Sorimachi. Basic characteristics of a fractance device. IEICE Transactions on Fundamentals of Electronics, Communications and Computer Sciences, 75(12):1814-1819, 1992.

[45] JM Patin. A very short proof of stirling's formula. Amer. Math. Monthly, 96(1):41-42, 1989.

[46] John PJ Pinel. Biopsychology. Pearson Boston, MA, 2011.

[47] Rafael Fernandes Pinheiros. O problema de Lurie e aplicações às redes neurais. PhD thesis, Universidade de São Paulo, São Paulo, 2014.

[48] Igor Podlubny. Fractional differential equations: an introduction to fractional derivatives, fractional differential equations, to methods of their solution and some of their applications, volume 198. Elsevier, Amsterdã, 1998.

[49] Harry Pollard. The completely monotonic character of the Mittag-Leffler function. Bulletin of the American Mathematical Society, 54(12):1115-1116, 1948.

[50] Herrmann Richard. Fractional Calculus: An Introduction for Physicists. World Scientific, Singapura, 2014.

[51] Antônio C. Roque. Introdução à neurociência computacional. USP, Ribeirão Preto, 2018. 
[52] Frank Rosenblatt. The perceptron: a probabilistic model for information storage and organization in the brain. Psychological review, 65(6):386, 1958.

[53] Stefan G Samko, Anatoly A Kilbas, and Oleg I Marichev. Fractional Integrals and Derivatives: Theory and Applications. CRC Press, Boca Raton, 1993.

[54] Jean-Jacques E Slotine, Weiping Li, et al. Applied Nonlinear Control, volume 199. Prentice hall Englewood Cliffs, NJ, 1991.

[55] Junio C A Soare. Cálculo Fracionário e as Equações de Evolução. PhD thesis, Unicamp, 2016.

[56] Anastasio Thomas. The fractional-order dynamics of brainstem vestibulo-oculomotor neurons. Biological cybernetics, 72(1):69-79, 1994.

[57] Johannes Weissinger. Zur theorie und anwendung des iterationsverfahrens. Mathematische Nachrichten, 8(1):193-212, 1952.

[58] Svante Westerlund and Lars Ekstam. Capacitor theory. IEEE Transactions on Dielectrics and Electrical Insulation, 1(5):826-839, 1994.

[59] Chen Yangquan. Ubiquitous fractional order controllers. In Proceedings of the 2nd IFAC Symposium on Fractional Derivatives and Applications (IFAC FDA06, Plenary Paper), pages 19-21, 2006.

[60] Shuo Zhang, YangQuan Chen, and Yongguang yu. A survey of fractional-order neural networks. In Volume 9: 13th ASME/IEEE International Conference on Mechatronic and Embedded Systems and Applications, page V009T07A024, 082017.

[61] Shuo Zhang, Yongguang Yu, and Hu Wang. Mittag-leffler stability of fractional-order Hopfield neural networks. Nonlinear Analysis: Hybrid Systems, 16:104-121, 2015. 